\title{
AN INVESTIGATION OF THE RELATIONSHIP BETWEEN SLEEP AND FATIGUE IN THOSE WITH AND WITHOUT INSOMNIA \\ by
}

\author{
Andrea L. Harris \\ Master of Arts, Ryerson University, 2010 \\ Honours Bachelor of Arts, The University of Western Ontario, 2007
}

\author{
A dissertation \\ presented to Ryerson University \\ in partial fulfillment of the \\ requirements for the degree of \\ Doctor of Philosophy \\ in the program of \\ Psychology \\ Toronto, Ontario, Canada, 2014 \\ (C) Andrea L. Harris 2014
}




\section{AUTHOR'S DECLARATION}

I hereby declare that I am the sole author of this dissertation. This is a true copy of the dissertation, including any required final revisions, as accepted by my examiners.

I authorize Ryerson University to lend this dissertation to other institutions or individuals for the purpose of scholarly research.

I further authorize Ryerson University to reproduce this dissertation by photocopying or by other means, in total or in part, at the request of other institutions or individuals for the purpose of scholarly research.

I understand that my dissertation may be made electronically available to the public. 


\begin{abstract}
Insomnia

Doctor of Philosophy, 2014

Andrea L. Harris

Psychology

Ryerson University
\end{abstract}

An Investigation of the Relationship between Sleep and Fatigue in Those With and Without

There is currently mixed evidence for the relationship between poor sleep and daytime fatigue. It is well documented that retrospective measures of insomnia and fatigue are highly correlated with one another. However, other studies fail to demonstrate a link between objectively less sleep and fatigue; that is, individuals with shorter sleep times do not necessarily report increased fatigue. As such, the relationship between these two constructs remains unclear. The current investigation will help to elucidate the complex relationship between sleep and fatigue among those with and without insomnia by advancing the existing literature in two important ways. First, this study proposed to examine the temporal relationship between sleep and fatigue across two weeks, thereby investigating whether sleep and fatigue occur in accordance with one another over time. Second, this study utilized a multi-method approach by collecting subjective (i.e., sleep diary) and objective (i.e., actigraphy) measures of sleep, as well as retrospective (i.e., visual analogue scales: VAS) and prospective (i.e., momentary ratings) measures of fatigue. Two separate hierarchical linear models were used to test whether sleep (measured by sleep quality and total sleep time) predicted daytime fatigue on the VAS and actigraph, respectively. The secondary objective asked whether cognitive-behavioural variables (i.e., maladaptive sleep beliefs, fear and avoidance of fatigue, and fatigue-based rumination) may help account for the 
relationship between sleep and fatigue using mediation. The results of the primary analyses suggested that sleep quality significantly predicted VAS fatigue ratings, whereas total sleep time was a significant predictor of fatigue within- but not between-persons. No significant relationships were found between objective measures of sleep and momentary fatigue ratings. Finally, each of the cognitive-behavioural variables, with the exception of avoidance of fatigue, were significant mediators of the relationship between sleep and fatigue. The results demonstrated that compared to sleep quantity, our perception of sleep may play a more important role in predicting reports of daytime fatigue. These findings could help decrease the burden that individuals with insomnia place on their total sleep times, and instead, treatment could focus on challenging maladaptive sleep-related cognitions, which ultimately could lessen the overall sleep-related anxiety. 


\section{Acknowledgments}

I would like to thank my graduate supervisor and research mentor, Dr. Colleen Carney, for her invaluable guidance, support, and mentorship throughout my graduate training as well as the preparation of this dissertation. Working alongside Colleen in the Sleep and Depression (SAD) lab has been a formative experience in my development as a competent researcher and clinician. Across my varied experiences in the SAD lab, I have had the opportunity to work towards establishing my proficiency as a sleep researcher focusing on insomnia and related psychological disorders. Through her fostering of autonomy and independence in research, Colleen has encouraged me to develop and refine a unique research program focusing on the experience of fatigue within insomnia. In addition to her expertise in the insomnia literature, Colleen's excellent instruction in scientific research methodology and her dedication to being both a teacher in addition to a researcher have helped me be successful in the completion of this project.

I would also like to thank Dr. Janice Kuo for her involvement in the development of this project, as well as for the valuable feedback she provided me throughout the final stages of the writing process. I am certain that this helpful feedback strengthened this dissertation and its contribution. Furthermore, thank you to the members of my dissertation examining committee, Dr. Traci McFarlane, Dr. Elizabeth McCay, and Dr. Catherine Fichten. I appreciate you taking the time and energy to be involved in this project and for taking an interest in my research.

The research assistants, volunteers, and project managers in the SAD lab have contributed to this project in a variety of important ways, including recruitment, screening participants, and inputting and scoring data. I want to thank them all for their invaluable assistance throughout the data collection portion of this project. I would especially like to thank 
my two lab mates, Taryn and Angela, who have been involved with this project since its inception. Their unwavering support and encouragement throughout graduate school and our work in the SAD lab has been greatly appreciated.

I sincerely want to thank my graduate school cohort for their support and close friendship throughout the duration of our graduate studies. My classmates have helped to make this journey a very enjoyable and memorable experience, and I am so grateful to have made both lifelong friends and a close network of future colleagues. Finally, thank you to my husband, parents, family and friends for their unconditional love and support throughout the completion of this dissertation and my graduate studies. 


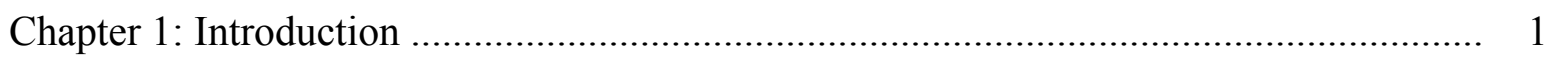

1.1 Review of Insomnia Literature...................................... 2

1.2 Review of Fatigue Literature................................... 9

1.3 The Relationship between Sleep and Fatigue in Insomnia................ 14

1.3.1 Challenges with respect to the Measurement of Sleep and Fatigue....... 16

1.3.2. The Relationship between Subjective and Objective Sleep Impairments with Fatigue ....................................................................... 19

1.3.3 The Relationship between Sleepiness and Insomnia................. 21

1.4 What else could account for the Relation between Sleep and Fatigue........ 24

1.4.1 Maladaptive Sleep Beliefs..................................... 26

1.4.2 Fear and Avoidance of Fatigue................................... 27

1.4.3 Fatigue-Based Rumination .................................... 29

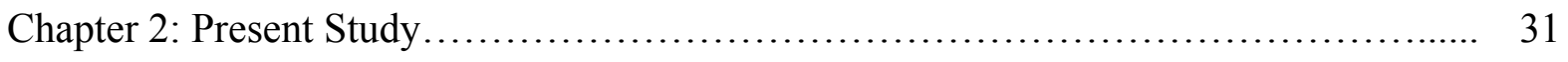

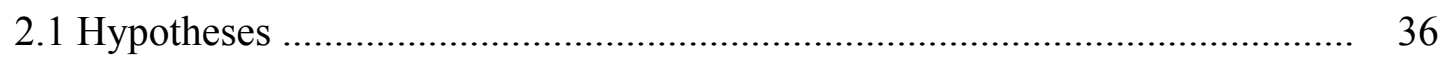

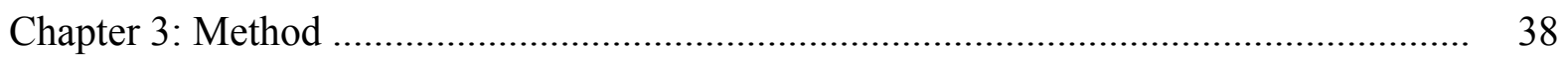

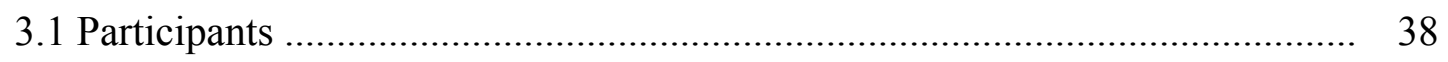

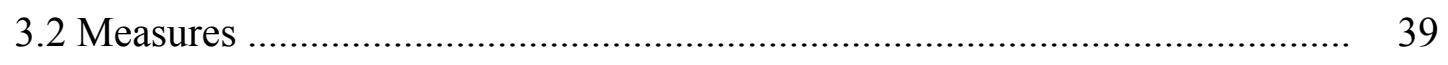

3.2.1 Diagnostic Interviews........................................ 39

3.2.2 Outcome Measures........................................... 40

3.2.3 Self-Report Questionnaires................................... 43

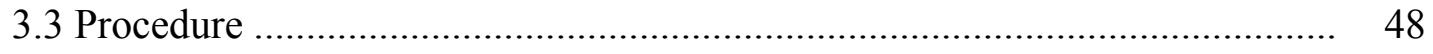

3.4 Statistical Analyses................................................... 49

Chapter 4: Results.................................................. 52 
4.1 Participant Demographic Characteristics........................... 52

4.2 Hierarchical Linear Modelling................................... 59

4.2 .1 Sleep Diary............................................ 61

4.2 .2 Actigraphy................................................ 65

4.2 .3 Group Differences........................................ 66

4.3 Mediation Analyses............................................... 68

4.3.1 Simple mediation model................................... 68

Chapter 5: Discussion................................................. 69

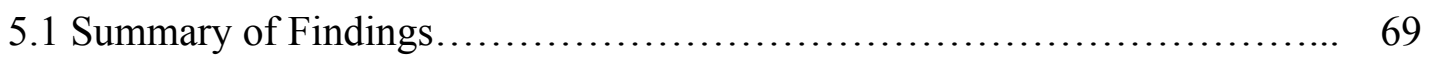

5.2 Theoretical and Clinical Implications.............................. 76

5.3 Study Strengths and Limitations................................ 80

5.4 Summary and Conclusions...................................... 87 


\section{List of Tables}

Table 1: Participant Demographics for Normal Sleepers, Insomnia Disorder, and the Total Sample ......................................................... 53

Table 2: Means, Standard Deviations, and Cronbach's Alphas for Self-Report Measures 55

Table 3: Correlations among Self-Report Measures for the Total Sample.............. 56

Table 4: Means and Standard Deviations Scores on Sleep Diary and Actigraphy

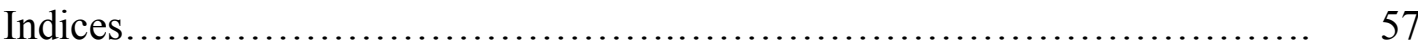

Table 5: Correlations among Sleep and Fatigue Indices on the Sleep Diary and Actigraphy for the Total Sample........................................ $\quad 58$

Table 6: Final Model of Sleep Diary Total Sleep Time and Sleep Quality Predicting

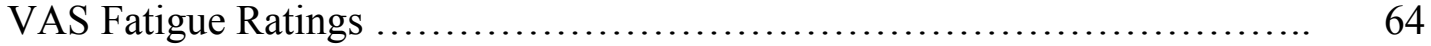

Table 7: Final Model of Actigrpahy Total Sleep Time and Fatigue Ratings on the Actigraph............................................................ 


\section{List of Appendices}

Appendix A: Sleep Diary ….................................................... 88

Appendix B: Visual Analogue Scale (VAS) .............................. 89

Appendix C: Fear and Avoidance of Fatigue Scales (FOF/AOF) $\ldots \ldots \ldots \ldots \ldots \ldots \ldots$.

Appendix D: Daytime Insomnia Symptom Response Scale (DISRS)............. 91

Appendix E: Consent Agreement for Insomnia Disorder..................... 92

Appendix F: Consent Agreement for Normal Sleepers ....................... 97 


\section{CHAPTER 1}

\section{INTRODUCTION}

Insomnia is a devastating disorder and a significant public health concern which is diagnosed based on a subjective complaint of difficulty initiating sleep, difficulty maintaining sleep, early-morning awakenings, or feeling poorly rested despite an adequate amount of sleep (American Psychiatric Association, 2013). The symptoms must be present for at least three months and cause clinically significant distress and impairment in social or occupational domains. Specifically, insomnia often results in daytime complaints, including those of fatigue, difficulty concentrating, and mood disturbances (American Psychiatric Association, 2013). In fact, the most prominent daytime complaint among people with insomnia is fatigue (Kales et al., 1984; Kloss, 2003; Moul, Nofzinger, Pilkonis, Houck, Miewald, \& Buysse, 2002) and this adverse daytime experience is one of the foremost reasons for seeking help from a health practitioner. Daytime fatigue can be defined as weariness, weakness, or depleted energy, which can significantly interfere with daytime functioning (Pigeon, Sateia, \& Ferguson, 2003). Despite the prominence of fatigue, the focus in insomnia has been on nighttime symptomatology, to the exclusion of the daytime sequelae. That is, while those with insomnia are consistently complaining of daytime fatigue, little attention has been paid to daytime impairments of insomnia in both clinical and research settings. For example, cognitive behavioural therapy for insomnia (CBT-I) does not include a module which addresses daytime impairments directly, and a review of the CBT-I literature concluded that there is limited evidence of improvements in daytime functioning after undergoing treatment for insomnia (Morin, Bootzin, Buysse, Edinger, Espie, \& Lichstein, 2006). In addition, the nighttime symptoms of insomnia have been explored more extensively in the research literature as compared to daytime effects. The lack of research 
and treatment focus on daytime symptoms, such as fatigue, is particularly troubling given the prominence and distress associated with the complaint in those with insomnia, as well as the fact that our treatments do a suboptimal job in alleviating fatigue. Thus, this study focuses on the important endeavor of understanding fatigue.

\subsection{Review of Insomnia Literature}

Epidemiological studies indicate that insomnia is a widespread health condition affecting approximately $10-15 \%$ of the adult population (Ford \& Kamerow, 1989; Ohayon, 2002). Other estimates suggest that up to $30 \%$ of adults from varying ethnicities across the world have at least one of the symptoms of insomnia (Roth, 2007). In addition, chronic insomnia is associated with reduced quality of life, increased healthcare utilization, increased use of prescription medication, increased absenteeism, decreased work productivity and increased traffic and work-related accidents (Edinger \& Wohlgemuth, 1999; Roth \& Ancoli-Israel, 1999). Notably, the economic costs to Canadians are substantial, with total annual costs in the province of Quebec alone estimated to be $\$ 6.5$ billion (Daley, Morin, LeBlanc, Gregoire \& Savard, 2009). Specifically, annual costs for Canadians associated with decreased work productivity are estimated to be $\$ 5$ billion, and the annual per-person cost for individuals with the disorder is estimated at $\$ 5010$ (Daley et al., 2009). Furthermore, insomnia can often exacerbate symptoms of other psychiatric disorders, including depression and anxiety (Ohayon, Caulet, \& Lemoine, 1998), and these comorbid groups can be particularly challenging to treat (Smith, Huang, \& Manber, 2005).

As insomnia is primarily associated with disturbances of sleep, it is important to understand the mechanisms regulating sleep in all individuals, both with and without insomnia. There are two primary processes which regulate sleep: the homeostatic and circadian systems (Saper, Cano, \& Scammell, 2005; Webb, 1988). The homeostatic system controls the propensity 
to produce sleep (i.e., sleep drive) by building "pressure" to sleep as the number of hours of wakefulness increase (i.e., sleep debt). This pressure is released during sleep and begins to build upon awakening. The circadian system affects the timing of the sleep-wake schedule in the 24hour day. In normal sleepers, the homeostat and circadian systems align, such that "sleepy" cues due to prolonged daytime wakefulness correspond to the fading of alerting signals from the clock to allow for sleep. When alerting signals are emitted from the clock the next day in the morning hours, there is no competing sleep debt and the individual can start their day with some degree of alertness. The environment also plays a role in regulating sleep, in the sense that sleep-related stimuli, such as the bed and bedroom environment are paired regularly with good sleep, and thus condition the individual to be ready for sleep. Thus, good sleep is obtained quite automatically for the normal sleeper, and effort is unnecessary to obtain these results (Espie, 2002).

Just as the homeostatic and circadian mechanisms can help us appreciate how normal sleep unfolds, so too can they help us understand how poor sleep develops. For example, behaviours such as sleeping-in in the morning, going to bed early, or staying in bed during the day, can result in lowered homeostatic drive for sleep, and thus hinder the production of good sleep at night (Pigeon \& Perlis, 2006). In addition, habits such as varying bed times and rise times can disrupt the circadian system, just as in jet lag, wherein the clock on the wall does not match the internal clock in our bodies. Finally, while the circadian and homeostatic systems often work hand-in-hand, both systems can be trumped by a third process, the arousal system, which is considered to have a central role in insomnia (Bonnet \& Arand, 1995; Espie, Broomfield, MacMahon, Macphee, \& Taylor, 2006). Even during periods of optimal homeostatic drive and timing of sleep, if the body perceives a threat in the environment, the arousal system will keep the individual awake to address the threat. In these situations, natural associations 
between the sleep disturbances and the bedroom unintentionally condition the bed to be an arousing/wakeful stimulus, which directly opposes the intended result of the bed being a cue for sleepiness.

One might still wonder how chronic insomnia develops. The development of this condition is primarily dependent on three factors: predisposing factors, precipitating events, and perpetuating factors (Spielman \& Glovinsky, 1991). Predisposing factors are individual characteristics that can place an individual at increased risk for developing insomnia. These can include cognitive factors, such as the propensity to worry (Lichstein \& Rosenthal, 1980), or biological factors, such as sensitivity to light (Chesson et al., 1999). They may also include demographic characteristics; for example, it is well established that females and older individuals are at increased risk for the development of insomnia (Ohayon, Carskadon, Guilleminauld, \& Vitiello, 2004; Zhang \& Wing, 2006). In addition, those with comorbid medical disorders (Katz \& McHorney, 1998) or psychiatric disorders (Ford \& Kamerow, 1998) are more likely to suffer from insomnia. When such predisposing factors are present and come into contact with stressful life events (e.g., precipitating factors), the individual may develop an acute sleep disturbance (Healy, Kales, Monroe, Bixler, Chamberlin, \& Soldatos, 1981; Morgan \& Clarke, 1997). As a result of the distress associated with this sleep impairment, some individuals may engage in thought processes and behaviours in order to help cope with their temporary sleep disturbance, such as going to bed early or taking a sleeping pill. In fact, it is these cognitive and behavioural perpetuating factors that will ultimately turn their acute sleep disturbance into chronic insomnia. Furthermore, as discussed above, these perpetuating factors have negative impacts on the homeostatic and circadian systems (Bootzin, 1972; Harvey, 2002), and it is the interaction 
between such perpetuating factors and sleep regulatory processes which works to maintain the insomnia.

There is ample evidence that sleep-incompatible behaviours are key contributors to the maintenance and perpetuation of insomnia. Indeed, insomnia sufferers tend to have poor bedtime habits and do not maintain consistent sleep-wake schedules (Bootzin, 1972). For example, those with insomnia often attempt to catch-up on sleep or manage fatigue in the day by napping or sleeping-in (Morin, 1993). As explained above, while these strategies appear to be effective in the short-term, they interfere with the body's homeostatic sleep drive, which is designed to compensate for accumulated sleep loss by increasing sleep depth (Feinberg, March, Floyd, Jimison, Bossom-Demitrack, \& Katz, 1985; Webb, 1988). Furthermore, according to the stimulus control theory of insomnia (Bootzin, 1972), spending excessive time in the bedroom during daytime hours can create stimulus dyscontrol. That is, pairing sleep-related stimuli (i.e., the bedroom) with wakeful activities can lower the likelihood that the bedroom will elicit the desired response of sleepiness during the night. Other maladaptive behaviours, such as being sedentary during the day (Morgan, 2003; Sherrill, Kotchou, \& Quan, 1998) or engaging in mentally stimulating activities during the pre-sleep period (Broman \& Hetta, 1994), may also increase sleep onset latency. These maladaptive sleep behaviours can come to perpetuate one another, thereby contributing to the onset and maintenance of the insomnia problem.

One might wonder how such disruptive sleep habits develop. One possibility is that the thinking patterns and general cognitive styles of those with insomnia, including unhelpful beliefs about sleep (Morin, Stone, Trinkle, Mercer \& Remsberg, 1993), may underlie these maladaptive sleep behaviours. For example, some individuals believe that they cannot cope with sleep loss. After a poor night's sleep, they may then decide to engage in behaviours, such as sleeping-in or 
napping, in order to compensate for this sleep loss. However, as discussed above, "catching up" on sleep during the day can disrupt the body's homeostatic drive, which can then make it more difficult to produce deep, continuous sleep at night. Sleeping during the day can also make the bedroom become a trigger for wakefulness during the night. Thus, it is likely that cognitions support and maintain the sleep-disruptive habits, which in turn perpetuate the insomnia.

Given the influential role of cognitive processes, insomnia theorists have begun to delineate how these mechanisms work to perpetuate the disorder. As one example, Harvey's (2002) cognitive model of insomnia posits that individuals with sleep difficulties experience negatively toned mental activity as a result of their poor sleep. These negative thought processes can induce a state of heightened arousal and distress, which increases attentional bias towards threatening stimuli, whereby poor sleepers focus their attention on distressing sleep-related cues (i.e., threats). As a result of these negative thoughts about sleep and attentional bias, they selectively monitor for sleep-related threats both internally and externally (in the environment). The increased sleep-related anxiety together with the bias toward sleep threat can actually make it more difficult to fall asleep, as other research has shown that the optimal conditions for sleeponset include minimal cognitive activity and effort in the pre-sleep period (Espie, 2002; Kohn \& Espie, 2005). In general, the model suggests that the aforementioned cognitive processes trap the individual in an ongoing cycle, wherein they become more absorbed by and anxious about their sleep difficulty. Fichten and colleagues (1998) also propose a cognitive model of insomnia, which emphasizes negative thoughts during nocturnal wakefulness. They posit that nighttime negative cognitions can precipitate other maladaptive nocturnal behaviours (e.g., tossing and turning) and faulty perceptions (e.g., overestimation of time spent awake in bed). These 
processes are thought to interfere with the ability to maintain or return to sleep, thereby perpetuating the sleep problem (Fichten, Libman, Creti, Amsel, Tagalakis, \& Brender, 1998).

Indeed, other theorists suggest that cognitive processes play a central role in the maintenance of insomnia. Two theoretical models in particular emphasize the processes involved in normal sleep, which are disrupted in those with insomnia. For example, Espie's psychobiological inhibition model (2002), or the more recent attention-intention-effort pathway (Espie et al., 2006), posit that intrusive thinking in the pre-sleep period inhibits the normal reductions in arousal necessary for sleep to unfold. In particular, selective attention to sleeprelated stimuli, intention to sleep, and putting forth effort in the pre-sleep period all work together to disrupt the normal sleep-wake cycle. Similarly, Lundh and Broman's (2000) theory of sleep-interfering and sleep-interpreting processes suggests that the interaction of stressful life events, arousal, and negative appraisal of sleep conspire to produce insomnia. Many theories about insomnia focus on sleep-interfering processes, which include traumatic and stressful life events, the resulting arousal of which is presumed to contribute to the sleep disturbance. However, Lundh and Broman argue that sleep-interpreting processes, which include perception of and beliefs about sleep, appraisals of one's sleep, and attributions concerning good and poor sleep, also play an integral role in the maintenance of insomnia. Indeed, maladaptive beliefs about sleep, which include negative appraisals and unhelpful attributions of poor sleep, differentiate those with and without insomnia (Carney \& Edinger, 2006). Thus, according to these cognition-based insomnia models, cognitive processes are key in the maintenance of the insomnia problem and thus are important targets of research.

Hyperarousal models of insomnia argue that people with insomnia have elevated arousal levels throughout the 24-hour period. Studies exploring this hypothesis have found increased 
physiological activation in those with insomnia, such as increased heart rate and elevated core body temperature (Monroe, 1967). Researchers examining event-related potentials have shown increased sensitivity to auditory stimuli among people with insomnia during wake and sleep onset (Reimann et al., 2010). Interestingly, poor sleepers have more difficulty falling asleep during the day than their good sleeper counterparts, suggesting that in fact this increased arousal operates both at night and during the day (Bonnet \& Arand, 1995; Edinger et al., 1997; Edinger, Means, Carney \& Krystal, 2008). Indeed, one key study in the hyperarousal literature demonstrated that by administering $400 \mathrm{mg}$ of caffeine three times daily to normal sleepers, thereby artificially inducing a state of heightened arousal, symptom reports were similar to those who have insomnia both at night and during the day (Bonnet \& Arand, 1992). Taken together there is widespread support for the hyperarousal model and general consensus in the field that people with insomnia are more highly aroused across the 24-hour period than their good sleeper counterparts.

Other models of insomnia focus on its biological underpinnings. Several of these models have explored the relationship between sleep indices and the HPA (hypothalamic-pituitaryadrenal) axis, and often focus on degree of cortical secretion over the course of the day. In normal sleepers, cortisol increases in the hour following wakening and is followed by a steep decline over the late morning hours; cortisol then gradually declines over the course of the day. Studies have shown that sleep loss can increase cortisol secretion in the evening hours as well as during the night (Leproult, Copinschi, Buxton, van Cauter, 1997), a time during which cortisol levels are otherwise found to be quite low. Other studies have found that cortisol secretion upon awakening was lower in the morning hours and correlated negatively with wakefulness during the night (Backhaus, Junghanns \& Hohagen, 2004). Interestingly, the literature on insomnia and 
HPA axis activity may help to explain the high rate of comorbidity between insomnia and depression; recent evidence suggests that approximately $60 \%$ of people with depression are found to have elevated cortisol secretion (Reimann et al., 2010). Further research in this area may help to uncover one possible pathway that leads people with insomnia to be at increased risk for the development of depression.

The majority of the mechanisms involved in the maintenance and perpetuation of insomnia mentioned above are, as would be expected, directly or indirectly related to sleep. For example, sleeping during the day, believing that you need a certain number of hours of sleep, and monitoring for sleep-related threats are all aforementioned maladaptive behaviours and cognitions that make insomnia more likely to occur. Indeed, the primary symptoms associated with insomnia comprise some form of sleep disturbance, as these are the symptoms required in order to warrant a diagnosis. In fact, the term insomnia has become synonymous with poor sleep, in the sense that the terms "poor sleepers" and "people with insomnia" are used interchangeably in the literature. However, it has become clear over the years that insomnia comprises more than complaints of poor sleep: there are additional sequelae that impair the daytime performance of individuals with insomnia. Indeed, more recent research has begun to explore the daytime consequences of insomnia and sleep complaints, and this research has focused primarily on daytime fatigue.

\subsection{Review of Fatigue Literature}

Whereas insomnia is most commonly associated with nighttime sleep impairments, it is more accurately described as a 24-hour disorder (Bonnet \& Arand, 1995; Buysse et al., 2007; Harvey, 2002; Moul et al., 2002; Riedel \& Lichstein, 2000). Daytime symptoms of insomnia include fatigue, difficulty concentrating, mood disturbances, and loss of motivation (American 
Psychiatric Association, 2013). These daytime impairments can exert adverse effects on those with insomnia with regard to social and occupational functioning. For example, people with insomnia are more likely to be distressed about their personal lives (Moul et al., 2002), and rate their quality of life lower than those without sleep problems (Roth \& Ancoli-Israel, 1999). In addition, people with insomnia report greater difficulties performing in the workforce (Leger et al., 2002) and have higher rates of absenteeism (Ozminjowski, Wang, \& Walsh, 2007). With respect to their daytime psychological functioning, individuals complaining of insomnia have higher rates of fatigue, depression, anxiety, irritability and cognitive impairments (Riedel \& Lichstein, 2000; Shekleton, Rogers, \& Rajaratnam, 2010). In accordance with these research findings, the International Classification of Sleep Disorders (ICSD) as well as the Diagnostic and Statistical Manual of Mental Disorders (DSM-5) and the Research Diagnostic Criteria (RDC) now require that a complaint of daytime impairment be present in order to justify a diagnosis of insomnia (American Psychiatric Association, 2013; American Academy of Sleep Medicine, 2005). Thus, there is a current consensus in both the literature and the diagnostic nosologies that in addition to difficulties sleeping throughout the night, insomnia also has significant daytime sequelae.

Before we can understand the role of fatigue in insomnia, it is important to consider the mechanisms that underlie fatigue in other subgroups and in the general population. Indeed, fatigue is a common symptom of many neurological disorders (e.g., multiple sclerosis, anemia), psychiatric disturbances (e.g., depression, somatoform disorder), and is a disorder in and of itself (i.e., chronic fatigue syndrome). Fatigue can be distinguished as either peripheral or central. Peripheral fatigue is purely a physical condition, which can be directly attributable to failure of neuromuscular function, metabolic defects, or peripheral circulatory failure. Central fatigue, on 
the other hand, can be associated with both physical and mental impairments, and can be defined as difficulty initiating or sustaining voluntary activity in the absence of objective cognitive or motor impairments (Chaudhuri \& Behan, 2000; 2004). This latter type of fatigue is what we find in insomnia.

According to Chaudhuri and Behan's (2004) conceptual model of central fatigue, determining the presence of fatigue involves considering whether there is sufficient effort in order to achieve a certain outcome or activity. If we think of applied effort as having a physiological set point, the determinants of whether this threshold is met may depend on several factors. Thus, degree of effort can be influenced by both internal (i.e., limbic system) and external (i.e., incentive) motivational input, or by feedback from sensory, motor and cognitive systems, which establishes level of perceived exertion. Using this conceptualization, pathological fatigue can be thought of as one or more changes to the systems regulating effort to carry out voluntary action (Chaudhuri \& Behan, 2000; 2004).

While the exact mechanisms underlying the role of fatigue in insomnia are yet to be determined, it is likely that the "feedback of perceived exertion", in combination with reduced applied effort, are contributing factors to the pathology. Specifically, as a result of the sleep dysfunction in insomnia, sufferers often believe they should do as little as possible in order to conserve energy and maximize rest; this is often perceived as the sensible way to combat the insomnia and the fatigue (Ree \& Harvey, 2004). As a result, they tend to overestimate their perceived level of exertion and suppress their effort to engage in further activities. Paradoxically, however, research suggests that increasing rest at the expense of engaging in activities is a major perpetuating factor of fatigue, as we know that expending energy leads to increased energy resources (Petruzzello, Hall, \& Ekkekakis, 2001; Puetz, O’Conner, \& Dishman, 2006). Thus, 
perhaps it is the perception of overexertion and the subsequent reduced effort to engage in activity that contributes to fatigue in those with insomnia.

Although these mechanisms remain to be tested empirically, the literature has demonstrated that daytime fatigue is both a very prevalent and deleterious symptom of insomnia (Buysse et al., 2007; Fichten, Creti, Amsel, Brender, Weinstein, \& Libman, 1995; Hauri \& Fisher, 1986; Hossain, Ahmad, Reinish, Hossain, \& Shapiro, 2005; Lichstein, Means, Noe, \& Aguillard, 1997; Means, Lichstein, Epperson, Johnson, 2000). In an epidemiological study, the most frequently cited reason for visiting a healthcare provider among insomnia sufferers was due to fatigue and other daytime pathologies related to the sleep disturbance, including anxiety and depression (Morin, LeBlanc, Daley, Gregoirre \& Merette, 2006). Further, a qualitative study using focus groups found that people with insomnia described daytime experiences as more problematic than nocturnal phenomena (Carey, Moul, Pilkonis, Germain \& Buysse, 2005). Participants in this study also believed that their insomnia was not well understood, and that understanding the daytime sequelae associated with insomnia is key to helping treat the problem. In accordance with these research findings, the 2005 consensus report on chronic insomnia stated that, "the importance of sleep disruption often rests with its impact on the individual's daytime function" (National Institutes of Health, 2005, p.5).

While complaints of fatigue are especially common among insomnia populations, rates of fatigue in good sleeper samples and the general population also warrant attention. It is estimated that $25 \%$ of all patients will complain about fatigue to their primary care physicians, and that daytime fatigue symptoms encompasses approximately 10 million visits to physicians per year (Kroenke, Wood, Manglesdorff, Meier, \& Powell, 1988). Indeed, research suggests that fatigue is a common complaint among the general population and otherwise healthy controls (Valko, 
Bassetti, Bloch, Held, \& Baumann, 2008; Walker, Katon \& Jemelka, 1993); in fact, some studies have documented comparable rates of fatigue in good and poor sleepers. Fichten and colleagues have demonstrated across several studies that a high level of distress is more important in predicting daytime fatigue than is poor versus good sleeper status (Fichten et al., 1995; Fichten et al., 1998). With respect to specific measures, the fatigue subscale on the Profile of Mood States (POMS) has often failed to differentiate those with and without insomnia (Bonnet \& Arand, 1995; Seidel et al., 1984). Further, studies examining the Minnesota Multiphasic Personality Inventory (MMPI; Hathaway \& McKinley, 1967) in insomnia have not found that the Hypomania scale, proposed to reflect activity and energy levels, distinguishes between good sleepers and those with insomnia (Bonnet \& Arand, 1995; Kales, Caldwell, Soldatos, Bixler \& Kales, 1983; Monroe, 1967; Seidel et al., 1984). Studies using the Fatigue Severity Scale (FSS), the most widely used fatigue-specific measure, have also suggested that fatigue is a common complaint among the general population (Valko et al., 2008; Walker et al., 1993). In particular, there has been debate as to the most effective cutscore on the FSS for determining severe fatigue, as the original cutscore of 4 (Krupp, 1989) would suggest that nearly half of a healthy sample would fall into this clinically significant category (Lerdal, Wahl, Rustoen, Hanestad, \& Moum, 2005). Taken together, while fatigue is a highly prevalent symptom of insomnia, there is also a considerable range and degree of fatigue found in healthy populations.

Perhaps the biggest difference between fatigue in those with and without insomnia is how individuals interpret and cope with fatigue. It is possible that those with insomnia are more likely to perceive fatigue negatively and have heightened awareness for detecting this aversive experience. Although many insomnia-based theories and models are focused on the sleep-related disturbances, cognitive models of insomnia (e.g., Harvey, 2002) take daytime impairments into 
consideration. For example, Harvey has proposed a cognitive model of daytime impairments in addition to nighttime impairments. In this sense, poor sleepers' heightened arousal and awareness of sleep-related stimuli during the night tends to mirror their increased attention to sleep-related cues during the daytime, which often manifests as fatigue. As an example, during the night, people may notice their heart pounding and thoughts racing, preventing them from falling asleep, whereas during the day, they may notice signs of fatigue or inability to concentrate, precluding their ability to function effectively. Indeed, the model further argues that monitoring for daytime symptoms of insomnia leads patients to overestimate the extent of their daytime impairment, and increases the likelihood that they will attribute these symptoms to their sleep problem. Thus, in both the night and daytime, this type of monitoring and detection of threatening cues continues to fuel sleep-related anxiety and worry, and thus the insomnia (Harvey, 2002).

While it is clear that daytime fatigue is a common symptom of insomnia, there is a dearth of research examining the role of fatigue in this condition. That is, while we have a decent understanding of the sleep disturbance characterizing those with insomnia, there continue to be many unanswered questions with respect to the role of daytime fatigue. Perhaps one reason for this gap in the literature, as some researchers have suggested, is that fatigue is assumed to be simply caused by the lack of sleep (Fortier-Brochu, Beaulieu-Bonneau, Ivers, \& Morin 2010; Riedel \& Lichstein, 2000). That is, fatigue is a presumed epiphenomenon of the more primary sleep disturbance. One notable implication of this supposed causality is that once the sleep problem is treated, so too would the daytime fatigue relent. However, there is not yet sufficient research on the nature of fatigue itself, nor the relationship between sleep disturbance and fatigue, to formulate such an opinion. For example, when students with insomnia were treated 
for their sleep condition, they found that while their sleep improved, their daytime impairments, including fatigue, did not abate with treatment (Means et al., 2000). The authors suggested that the relationship between daytime dysfunction and sleep impairment is a complex one, and more research is needed to determine the direction of causality, if in fact there is a causal relationship. Indeed, the subsequent section will reveal that even the basic association between sleep and fatigue in those with insomnia remains uncertain and requires further investigation.

\subsection{The Relationship between Sleep and Fatigue in Insomnia}

Poor sleep and daytime fatigue are often considered to be an intertwined problem. That is, there is a conception that poor sleep will inevitably lead to daytime fatigue. For example, research in our own lab has shown that both good and poor sleepers are more likely to attribute their daytime fatigue to poor sleep rather than other possible, even probable, causal factors of fatigue, such as illness, dehydration and boredom (Harris \& Carney, 2012). Other research has found that individuals with insomnia made stronger attributions of daytime impairments to poor sleep as compared to good sleepers (Morin et al., 1993). Such notions stem from societal and media influences, as well as research which suggest that obtaining good quality sleep is an effective way to counteract the experience of fatigue (Aaronson et al., 1999; Aaronson, Pallikkathayil, \& Crighton, 2003).

In line with this idea, there is considerable correlational support for a relationship between reported sleep disturbance and fatigue. Indeed, despite the notable levels of fatigue in the general population, many studies have found that people with insomnia report significantly higher levels of fatigue, compared to those without insomnia diagnoses (Buysse et al., 2007; Hauri \& Fisher, 1986; Hossain et al., 2005; Lichstein et al., 1997; Means et al., 2000). Researchers have used a variety of self-report measures in order to assess for fatigue, including 
the FSS, the Multidimensional Fatigue Inventory (MFI), the Daytime Insomnia Symptom Scale (DISS), and rating scales such as Visual Analog Scales (VAS). In a recent study, reports of insomnia were found to predict daytime fatigue on the FSS, after controlling for demographic, health, and quantitative sleep variables, such as sleep onset latency, total sleep time, and sleep efficiency (Ustinov et al., 2010). As noted in the previous section, qualitative data concur that complaints of fatigue are ubiquitous among people with insomnia and such daytime impairments are among the most distressing of insomnia symptoms. Taken together, the correlation between complaints of insomnia and daytime fatigue has been well established in the literature. While symptoms of fatigue are also found in healthy samples, the all-encompassing and selfperpetuating nature of fatigue in insomnia is noteworthy, and merits further investigation.

\subsubsection{Challenges with respect to the Measurement of Sleep and Fatigue}

While the studies mentioned above suggest a potential cross-sectional relationship between insomnia and fatigue, it remains unclear how sleep disturbance and fatigue are in fact related. As with many other areas of psychology, much of the sleep and fatigue research data has been aggregated into single scores in order to reduce error and increase ease of interpretation. As a result, findings may overlook an important aspect of the measurement of sleep and daytime indices: namely intra-individual variability. Intra-individual variability refers to the study of transient fluctuations in individual characteristics/behaviour over time (Hultsch, MacDonald, Hunter, Levy-Bencheton, \& Stauss, 2000; Knutson, Rathouz, Yan, Liu, \& Lauderdale, 2007). With respect to sleep variables in particular, it has been well-established that individuals with insomnia have more variability in their night-to-night sleep compared to good sleepers (Buysse et al., 2010; Coates et al., 1981; Edinger, Marsh, Mccall, Erwin, \& Lininger, 1991; Kay, Dzierzewski, Rowe, \& McCrae, 2013; Sanchez-Ortuno \& Edinger, 2012; Vallières, Ivers, 
Bastien, Beaulieu-Bonneau, \& Morin, 2005). This is likely due to the maladaptive sleep habits adopted by many individuals with insomnia, such as sleeping-in after a poor night of sleep or going to bed early the following night. Given that we know these attempts to "catch up" on sleep are rarely effective and in fact are often detrimental, this night-to-night variability can be particularly distressing for insomnia sufferers.

Fundamental to the concept of intra-individual variability is the importance placed on the variability of sleep within a given individual, in addition to between individuals. Within-person variables involve the fluctuations among one individual's sleep across time. For example, withinperson variation would involve comparing an individual's sleep on one night to their sleep on another night and exploring the differences between the two. By contrast, between-person variables correspond to the mean overall score (e.g., average number of hours of sleep) of that individual across time. Both within- and between-person variables are important to the study of sleep; however, traditional data analyses have been primarily concerned with differences between individuals, as well as between groups. Although the sleep literature is increasingly recognizing the importance of studying intra-individual variability of sleep, the majority of studies have explored this variability between groups (i.e., comparing sleep variability between poor sleepers and good sleepers). Given the consistent finding that poor sleepers have more variable sleep compared with good sleepers, a logical next step may be to better understand the within-person variability and further explore the dynamic relationship between sleep and other correlates which also fluctuate over time.

In line with these findings, it seems reasonable to explore the fluctuations of variables closely related to sleep, such as daytime fatigue, in order to better understand the nature of these relationships. Indeed, the exploration of within-person variables in the sleep literature has 
provided unique insights as to the relationship between sleep and its correlates. For example, nights characterized by lower ratings of sleep quality were found to be associated with reduced positive affect and higher negative affect the following day (McCrae et al., 2008). One study found that although between-person average ratings of total sleep time did not predict pain levels across two weeks, individuals who obtained more sleep compared to their average total sleep time on a given night reported decreased pain the following day (Dzierzewski et al., 2010) Similarly, it has also been found that higher within-person variability in sleep is associated with higher levels of stress independent of mean values (i.e., between-person variables) of these parameters (Mezick et al., 2009). No research to date has explored the intra-individual variability of sleep in association with fatigue; thus, understanding the temporal stability of the relationship between sleep and fatigue could shed some important insights into the nature of this relationship. For example, such an analysis could reveal whether poor sleep and fatigue vary in accordance with one another over time, or whether they are relatively independent of each other.

Interestingly, in McCrae et al. (2008) the finding that poor sleep had an effect on the subsequent day's mood only held true for subjective ratings of sleep quality, whereas objective indices of poor sleep did not demonstrate this effect. Thus, the manner in which specific constructs are measured (i.e., subjective versus objective, and retrospective versus prospective) warrants some attention. In addition to being cross-sectional, the measures of sleep and fatigue reviewed in the previous section primarily rely on self-report assessments. Fatigue in itself is a subjective construct, and thus self-report questionnaires are the foremost measure through which we are able to study fatigue (Aaronson et al., 1999). Sleep, on the other hand, has several validated methods of measurement, some of which are objective (e.g., polysomnography; actigraphy), prospective subjective (e.g., sleep diaries) and retrospective subjective (e.g., self- 
report). Polysomnography (PSG) records the physiological changes that occur during sleep whereas sleep diaries require participants to report on specific indices of their sleep upon awaking, such as sleep onset latency and number of nocturnal awakenings. However, according to the recommended standards for the diagnosis and assessment of insomnia (Buysse, AncoliIsrael, Edinger, Lichstein, \& Morin, 2006), insomnia is diagnosed based on a thorough sleep history indicating a subjective complaint of difficulty initiating or maintaining sleep. Thus, it is important to distinguish between insomnia, based on a subjective sleep complaint, and sleep disturbance, which may be based on quantitative or objective sleep impairments (Buysse et al., 2006).

According to the practice and research parameters in insomnia, PSG, although the goldstandard measure of objective sleep, is not recommended for the assessment of insomnia (Buysse et al., 2006; Chesson et al., 2000). This is because the symptoms of insomnia can be distinct from the objective manifestation of sleep disturbance. This distinction between subjective and objective sleep disturbances may help to elucidate the relationship between sleep and fatigue. For example, it may help us understand why some studies have been unable to find a direct connection between sleep loss and fatigue (Chambers \& Keller, 1993; Means et al., 2000). As a result, researchers have explored the possible differences between subjective and objective indices of poor sleep, and their relations with daytime impairments.

\subsubsection{The Relationship between Subjective and Objective Sleep Disturbance with Fatigue}

Given the subjective nature of the insomnia diagnosis, researchers have found discrepancies between reports of insomnia and quantitative measurements of sleep parameters. Indeed, some research has found that objective evidence of sleep impairment, such as PSG data, is not strongly associated with subjective sleep complaints (Buysse, Reynolds, Monk, Hoch, 
Yeager, \& Kupfer, 1991; Vitiello, Moe, \& Prinz, 2002). There are those who evidence objective poor sleep but do not complain of insomnia (Fichten et al., 1995; McCrae et al., 2003). There are also those who complain of insomnia but do not have objective evidence of poor sleep (McCrae et al., 2005); the diagnostic label for extreme cases of this phenomena is "sleep-state misperception" (American Sleep Disorders Association, 1997). This diagnostic category defines those who mistakenly perceive their sleep as wakefulness, and thus complain of insomnia without any objective indication of sleep impairments. Indeed, the existence of this subgroup in the ICSD suggests that some who endorse having sleep problems do not evidence poor sleep according to PSG and other objective indices.

As noted above, some studies have examined the differences between objective and subjective insomnia complaints and their respective relationships with daytime impairments. Such studies have compared three groups on their daytime functioning: distressed poor sleepers, non-distressed poor sleepers, and a good sleeper control group. While poor sleepers with and without distress did not differ significantly on sleep variables, poor sleepers experiencing high distress were more likely to complain of significant daytime impairments than their low distress counterparts (Alapin et al., 2000; Fichten et al., 1995). Most notably, in studies of these three groups in addition to a fourth group: complaining good sleepers (i.e., those with sleep-state misperception), this fourth group demonstrated higher levels of fatigue compared to noncomplaining poor sleepers (Fichten et al., 1995; McCrae et al., 2003; 2005). In one study, those with objective indicators of poor sleep were comparable on daytime functioning to controls whereas those with merely subjective complaints of poor sleep show impairments on daytime vigilance (Sugerman, Stern \& Walsh, 1985). Indeed, the finding that self-reported poor sleepers 
have greater daytime dysfunction than many of those with objective sleep deficits provides important insight into further understanding the relationship between sleep and fatigue.

Different methodologies have also been applied to further elucidate the differences between objective and subjective insomnia and their daytime symptoms. While the above mentioned studies looked at self-reported good and poor sleepers, the most relevant subgroup in the insomnia literature is that of objective insomnia. Those with only subjective complaints do not meet suitability criteria in clinical trials and psychoeducation is typically all that is needed to correct their mistaken perception of poor sleep. In an attempt to better understand the associated symptoms of insomnia using only one group of normal sleepers, individuals were yoked to follow an insomnia sleep pattern for one week (Bonnet \& Arand, 1996). In this yoked-control design, the electroencephalographic (EEG) sleep patterns of those with diagnosed insomnia were induced in a group of matched normal sleepers to help understand which insomnia symptoms would develop as a result of disturbed sleep. Given that the sleep produced in this sample was matched to those found in diagnosed insomnia, any differences in the outcome measures should purely reflect the changes in sleep. However, the associated daytime changes seen in this group were more reflective of mild sleep deprivation, and not consistent with the secondary symptoms reported among those with insomnia. That is, good sleepers induced with insomnia-based EEG sleep parameters demonstrated increased sleepiness which, as will be discussed in the subsequent section, is not often found in insomnia. This suggests that the secondary symptoms of those with insomnia, including daytime fatigue, are likely not directly related to poor sleep per se, and that instead other factors may be causing these daytime impairments. Indeed, other reviews agree that the magnitude of fatigue reports found among those with insomnia cannot be accounted for by the small degree of sleep loss found in these patients (Chambers \& Keller, 1993; Fichten, 
Libman, Bailes, \& Alapin, 2000; Riedel \& Lichstein, 2000). This idea will be discussed in further detail below.

\subsubsection{The Relationship between Sleepiness and Insomnia}

If poor sleep is the main contributing factor to daytime fatigue, it may follow that people with insomnia would also evidence more daytime sleepiness. Given the subjective nature and complexity of the fatigue construct, there is a tendency to view fatigue as synonymous with sleepiness. However, unlike fatigue, which is conceptualized as a state of weariness or lack of energy, sleepiness can be defined as the propensity to produce sleep at that moment (Pigeon et al., 2003). Perhaps one of the perceived benefits of considering fatigue and sleepiness as the same construct is that there is a well-known, objective measure of sleepiness: the multiple sleep latency test (MSLT). The test instructs participants to take four 20-minute naps throughout the day spaced out by two-hour intervals. Sleepiness is inferred based on whether and how quickly participants are able to fall asleep across the naps.

While there are temptations to lump fatigue and sleepiness into one construct, there are important differences between the manifestation of fatigue and sleepiness in those with insomnia. Overall, while there is considerable support for the relationship between insomnia and fatigue, there is opposing evidence for an association between insomnia and daytime sleepiness based on the objective MSLT (Edinger et al., 1997; Lichstein et al., 1994; Seidel et al., 1984; Sugerman et al., 1985). People with insomnia are actually less sleepy than their good sleeping counterparts (Bonnet \& Arand, 1995; Edinger et al., 1997; Edinger et al., 2008; Stepanski, Zorick, Roehrs, \& Roth, 2000). Other studies have examined the relationship between sleepiness and insomnia using subjective self-report measures, including the Epworth Sleepiness Scale (ESS) and Stanford Sleepiness Scale (SSS). Findings from this literature are mixed, with some 
studies suggesting that those with insomnia do not report elevated daytime sleepiness (Johns, 1991; Hossain et al., 2005; Lichstein \& Johnson, 1994; Lichstein et al., 1994; 1997; Seidel et al., 1984), and other studies documenting a positive relationship between insomnia and subjective sleepiness (Alapin et al., 2000; Edinger et al., 2008; Schneider, Fulda, \& Schultz, 2004).

The distinction between objective and self-report measures of sleepiness is an interesting one. It would appear that those with insomnia merely perceive elevated levels of sleepiness, when in fact these deficiencies do not manifest on the MSLT. Indeed, as discussed above with respect to cognitive theories of insomnia, a large portion of insomnia-related deficits are considered to be subjective in nature. Other researchers have simply suggested that the reduced sleep found among those with insomnia is not sufficient to produce objective increases in daytime sleepiness. As discussed above, this suggestion was put to the test by Bonnet and Arand (1996) when they artificially induced insomnia patterns into otherwise normal sleepers and did not find the usual daytime presentation seen among insomnia sufferers. To further test this prediction, a follow-up study explored the effects of reducing sleep in insomnia patients by $15 \%$ across one week (Bonnet \& Arand, 1998). Despite the reductions in sleep time, the patients did not demonstrate any objective evidence of increased sleepiness during the daytime, nor did they report elevated sleepiness on self-report measures. Thus, in conjunction with the findings presented in the previous section, these results further suggest that the sleep loss found in insomnia is not the sole contributor to the daytime impairments.

Of course, the question remains, if sleep loss in those with insomnia is not directly related to the numerous complaints of daytime fatigue, what else can account for this relationship? The following section will explore this question in depth. 


\subsection{What else could Account for the Relationship between Sleep and Fatigue in Insomnia?}

The abovementioned research has demonstrated that the relationship between sleep and fatigue may not be as clearly defined as previously thought. To summarize, it appears that while the association between retrospectively reported fatigue and insomnia complaints is relatively strong (Buysse et al., 2007; Lichstein et al., 1997; Means et al., 2000; Moul et al., 2002; Ustinov et al., 2010), the relationship between fatigue and objective indices of disrupted sleep is not as clearly supported (Bonnet \& Arand, 1996; 1998; Fichten et al., 1995; Fortier-Brochu et al., 2010). Thus, given that objective sleep loss is not associated with fatigue per se, an obvious question is what accounts for high levels of fatigue among those with insomnia? Some research points to the distress caused by insomnia, or perhaps the perception of poor sleep, as opposed to poor sleep itself, which can cause significant daytime impairment (Fichten et al., 1995; Ustinov et al., 2010). Indeed, recent research has suggested that poor sleep is likely not the only predictor of elevated fatigue in those with insomnia, and that research examining different pathways of relations between sleep and fatigue is warranted (Fortier-Brochu et al., 2010). One likely group of candidates which could underlie this relationship is cognitive-behavioural processes, which, as discussed in the first section, play an integral role in insomnia.

Theoretical models of insomnia have outlined how cognitive-behavioural mechanisms play an instrumental role in the perception of our sleep, and thus the perpetuation of insomnia. In particular, Harvey's (2002) cognitive model of insomnia suggests that processes such as attention, perception, safety behaviours and maladaptive beliefs can trap the patient into becoming increasingly more absorbed by and anxious regarding the insomnia. In addition, while Lundh and Broman (2000) propose that insomnia results from the interaction of sleep-interfering and -interpreting processes, sleep-interfering processes work to deprive the individual of sleep, 
and thus cannot alone explain insomnia, as we know that insomnia cannot be merely equated with sleep loss. Thus, perhaps it is the sleep-interpreting processes, such as appraisal and perception of sleep that fuel the insomnia and moreover, the daytime impairments. Taken together, in the context of these models, cognitive arousal and interpreting or attributional processes maintain insomnia, and thus may play an important role in explaining the relationship between sleep and fatigue.

Likewise, in Chaudhuri and Behan's (2004) model of central fatigue, fatigue is characterized as the failure to initiate and/or sustain physical or mental tasks requiring selfmotivation and applied effort. Perhaps it is the overestimation of perceived exertion and resulting reduced effort to engage in activity that contributes to the pathological fatigue found in insomnia. Indeed, maladaptive beliefs regarding lack of control over sleep or the need to conserve energy in order to combat the insomnia are possible factors which may explain this phenomenon (Ree \& Harvey, 2004). This attempt to conserve energy is carried out with the intention of reducing or avoiding fatigue, which according to those with insomnia, is a threatening experience (see Harvey, 2002). The more they ruminate about the negative consequences of fatigue, the more likely they are to disengage, with the hopes of conserving energy and feeling better. Thus, maladaptive beliefs about sleep and fatigue, negative appraisals of fatigue, and rumination about the causes of fatigue each contribute to this experience of fatigue. Further discussion of these processes, as well as how they can help explain the relationship between fatigue and insomnia, will be discussed in the remainder of this section.

In order to determine the role of a third variable in the relationship between an independent and dependent variable, tests of either moderation or mediation are proposed. Moderation analyses test whether a given factor affects the strength and/or the direction of the 
relationship between an independent variable (i.e., sleep) and dependent variable (i.e., fatigue).

Mediation analyses explore mechanisms which explain how or why the relationship between these two variables occurs (Barron \& Kenny, 1986; Muller, Judd, \& Yzerbyt, 2005). In the current study, mediation appears to be better suited to answer the overarching research question: namely, what accounts for the relationship between sleep and fatigue? Thus, the cognitivebehavioural variables discussed below will be proposed as potential mediators of the relationship between sleep and fatigue.

\subsubsection{Maladaptive Sleep Beliefs}

Given the etiologic role of cognitive processes in insomnia, and the possible contribution of cognitive factors to the experience of fatigue, perhaps the thinking processes and general cognitive styles of those with insomnia contribute to the relationship between sleep and fatigue.

We know that people with insomnia are characterized by maladaptive beliefs and worry regarding the consequences of sleep loss (Lichstein \& Rosenthal, 1980; Wicklow \& Espie, 2000). For example, poor sleepers tend to believe that low levels of energy during the daytime are a result of poor sleep (Carney \& Edinger, 2006; Morin et al., 1993). In fact, research suggests that poor sleep is but one of many causal factors that impact daytime energy, including boredom, dehydration, depression, and caffeine withdrawal (Harris \& Carney, 2012). As another example, people with insomnia often cancel activities and other obligations after a poor night's sleep in order to avoid the experience of fatigue. According to Harvey's (2002) model, holding these maladaptive, rigid beliefs combined with the reduced capacity to produce eight hours of sleep per night can create anxiety within the individual and continues to fuel the negative cognitive activity and the daytime impairment. Thus, unhelpful beliefs about sleep are additional 
exacerbating factors that work to further perpetuate the insomnia, and may be one possible mechanism through which we can explain the relationship between sleep and fatigue.

Indeed, research has found that poor sleepers tend to endorse stronger unhelpful beliefs and attitudes about sleep than do good sleepers (Carney \& Edinger, 2006; Morin et al., 1993). Given the important role that maladaptive sleep beliefs play in the perception of sleep and how it impacts daytime functioning, it is possible that these beliefs may help us understand the relationship between sleep and fatigue in those with insomnia. Thus, perhaps it is the rigidly held belief that poor sleep will inevitably lead to daytime fatigue that is the culprit, as opposed to the poor sleep itself directly causing the fatigue. In other words, it appears that prescribing to beliefs that poor sleep hinders daytime performance is what then makes it even more difficult to function during the day if a "good night of sleep" is not achieved. Indeed, this would explain why the relationship between sleep and fatigue in those with insomnia is largely subjective in nature. Thus, determining whether maladaptive sleep beliefs play a role in this relationship will provide further evidence that fatigue is unlikely a direct consequence of poor sleep. Given that anxiety features prominently in the contribution of maladaptive sleep beliefs to insomnia, anxiety was assessed in these analyses, in order to determine the direct contribution of these beliefs.

\subsubsection{Fear and Avoidance of Fatigue}

In addition to maladaptive beliefs about the consequences of poor sleep, there appears to be a cognitive vulnerability to the adverse experience of fatigue and other daytime symptoms among those with insomnia. Indeed, self-reported poor sleepers are more concerned by the prospect of fatigue than those without insomnia complaints (Harris \& Carney, 2009). More specifically, those with poor sleep had greater fear of fatigue as well as greater attempts to avoid the experience of fatigue. In other studies, people with insomnia are more preoccupied with 
sleep, and are more likely to attribute fatigue back to poor sleep than are good sleepers (Carney \& Edinger, 2006; Morin et al., 1993). Thus, it is clear that such individuals perceive fatigue as threatening and ascribe the existence of this negative mood state to their problematic sleep.

While this construct is newly emerging in the insomnia domain, these findings suggest that fear and avoidance of fatigue also may mediate the relationship between sleep and fatigue. Thus, perhaps it is the fearful anticipation of fatigue and the attempts to avoid this experience that lead to daytime impairments as opposed to the fatigue itself. Indeed, those who fear the consequences of poor sleep tend to monitor both their body sensations and daytime functioning for signs of fatigue; thus they are more likely to detect evidence of fatigue, as attention is drawn to the manifestations of drowsiness (Neitzert-Semler \& Harvey, 2007.). More generally, elevated fear of fatigue in those with insomnia also increases negatively toned cognitive activity in the pre-sleep period. It is this maladaptive thought process that is the entry point for Harvey's (2002) model, and interacts reciprocally with unhelpful safety behaviours. Attempts to avoid the experience of fatigue can be considered safety behaviours. For example, if one were to cancel their daytime activities due to anticipation of fatigue, their resulting inactivity and boredom will in turn exacerbate fatigue, and thus make the feared consequences of poor sleep more likely to occur. Thus, the fear of and attempts to avoid the fatigue experience may actually increase the detection of seemingly threatening fatigue stimuli, which can help to explain the association between poor sleep and fatigue. As is the case with maladaptive sleep beliefs, given the overlap between anxiety and constructs related to fear and avoidance, we will control for anxiety in these mediation analyses. 


\subsubsection{Fatigue-based Rumination}

Finally, rumination is a negatively-toned repetitive thought process that often focuses on past failure and is associated with depressed mood. This construct is often compared and contrasted with worry. In this sense, although both rumination and worry are considered to be repetitive thought processes (Segerstrom, Tsaw, Alden, \& Craske, 2000), they differ in terms of their cognitive content. Specifically, those who ruminate have a tendency to attribute their current mood state to past symptoms (Nolen-Hoeksema, 1991). By contrast, worry is more focused on the future, negative consequences of the current mood (Borkovec, Ray, \& Stober, 1998). While rumination has been researched primarily in the context of major depression, more recent research has demonstrated the importance of rumination in sleep. For example, research has found a significant association between rumination and subjective sleep quality (Thomsen, Mehlsen, Christensen \& Zachariae, 2003), and other findings suggest that rumination is more characteristic of poor sleepers compared to good sleepers (Carney, Edinger, Meyer, Lindman, \& Istre, 2006). Research from our lab confirms these findings, and also suggests that compared to worry, rumination plays as, if not more, of an important role in insomnia (Carney, Harris, Moss \& Edinger, 2010). These finding demonstrate the utility of examining rumination as a key cognitive process in insomnia.

In this regard, cognitive theorists in insomnia have acknowledged the importance of rumination, and propose that alongside worry, rumination is a key cognitive process that contributes to negative cognitive activity (Espie, 2002; Espie et al., 2006; Harvey, 2002; Lundh \& Broman, 2000). This argument is further supported by research indicating that rumination about sleeplessness is a vital component of cognitive activity in the pre-sleep period (Harvey, 2000; Wicklow \& Espie, 2000). In addition, Lundh and Broman (2000) consider rumination to 
be a sleep-interfering process; people who have a tendency to ruminate are predisposed to high arousal, which increases their likelihood of developing insomnia.

By way of building on the findings that rumination is important in insomnia, our lab has proposed a fatigue-based rumination scale, whereby the items reflect rumination about daytime insomnia symptoms (Carney, Harris, Falco \& Edinger, 2013). Given that rumination is pastfocused, we might expect that ruminative content in insomnia would be focused on the experience of fatigue, and that patients might look to the past (i.e., the previous night's sleep) to explain this daytime impairment. Preliminary findings suggest that insomnia is a significant predictor of rumination of daytime symptoms, even after controlling for depressed mood (Harris, Carney, \& Moss, 2010). Further research from our lab demonstrates that those with a cognitive vulnerability to insomnia tend to attribute their daytime fatigue back to poor sleep, as opposed to a myriad of other possible causes of fatigue (Harris \& Carney, 2012). This attribution tendency increases the perception that daytime fatigue is caused by the preceding night's poor sleep. Thus, ruminating about the daytime consequences of insomnia and the tendency to attribute fatigue to poor sleep may help explain the connection between sleep and fatigue in those with insomnia. Given that rumination is associated with low mood, we will assess for dysphoria in these mediation analyses. 


\section{CHAPTER 2}

\section{PRESENT STUDY}

The goal of the present study is to further elucidate the complex relationship between sleep and fatigue among those with and without insomnia. Although many correlational studies have found a positive association between fatigue and insomnia (Moul et al., 2002; Ustinov et al., 2010), it remains unclear how the sleep disturbance and fatigue are in fact related. That is, studies have failed to demonstrate a direct connection between objective sleep loss and fatigue. Moreover, the temporal relationship between fatigue and the myriad of sleep indices has not been studied to date. Thus, the mechanisms underlying the relationship between sleep complaints and fatigue require further exploration. In addition, while several cognitive variables have been proposed to mediate this relationship, these predictions remain to be tested empirically.

In order to further understand the complex relationship between sleep and fatigue, perhaps a different methodological approach must be taken. The majority of the studies discussed thus far have explored the association between sleep and fatigue via one score on a self-report fatigue scale and either an insomnia diagnosis or an average across nights using sleep diary data. Thus, most of the research to date in this area has been correlational and crosssectional. This is particularly true of fatigue data, as few, if any studies to date have tracked fatigue prospectively across time. Indeed, collecting sleep and fatigue data on a daily basis across time would advance our knowledge about the relationship between and variability among these two constructs. For example, although there may be a positive correlation between sleep and fatigue on self-report measures, this association may differ when repeated measurements of sleep and fatigue are being tracked across several weeks. Furthermore, intra-individual variability, as 
mentioned above, is a measurement technique which has been used to examine variability within a given individual, and can help us to further understand complex relationships which may vary over time. Given that we know the sleep of those with insomnia is highly variable, it would be important to explore the variability of sleep alongside fatigue, a construct about which we know very little. Thus, instead of having one value representing each person's sleep and fatigue, the current investigation proposes to examine the day-to-day fluctuations of sleep and daytime fatigue among those with and without insomnia. By exploring fluctuations in sleep alongside fatigue both within and between individuals, we can understand whether poor sleep and daytime fatigue occur in accordance with one another over time, or whether they are relatively independent phenomena.

This measurement approach will help to explore some unanswered questions and overcome some of the limitations in the current sleep and fatigue literature. For example, while it is clear that there is a correlation between poor sleep and fatigue, it will be interesting to learn whether this relationship holds true when the sleep and fatigue data are collected on a daily basis, across time. Further, with reference to intra-individual variability and the importance of testing these relationships within individuals, this investigation could help us understand whether one night of lower total sleep night for a given individual contributes to increased fatigue the following day, or whether daytime fatigue does not vary as a result of the previous night's sleep. Without being able to test a causal relationship between sleep and fatigue, understanding whether a poor night of sleep reliably leads to increased daytime fatigue will help us to understand the nature and dependence of this relationship. If it is found that daytime fatigue is relatively independent of poor sleep among those with insomnia, this finding helps us to challenge the widely held belief that poor sleep exclusively causes fatigue. Thus, while sleep and fatigue may 
be correlated, it is not necessarily the case that poor sleep directly leads to higher levels of daytime fatigue.

This study employed a multi-method approach using two weeks of prospective data, including both subjective and objective sleep and fatigue monitoring. Given that the literature has demonstrated important differences between objective and subjective indices of sleep (Buysse et al., 2006; Means, Edinger, Glenn, \& Fins, 2003), three measurements of sleep, each ranging in their degree of subjectivity and objectivity, were used. The most subjective measure of sleep was a rating of sleep quality, whereby participants rated the quality of their previous night's sleep on a scale from 1 (very poor) to 5 (very good). Participants made this rating on the sleep diary upon awakening in the morning. Total sleep time, as calculated by the sleep diary, was the second subjective sleep monitoring measure. Sleep diaries allow participants to record information about their sleep (e.g., time they got into bed, how long it took them to fall asleep etc.) upon awakening, and a simple algorithm allows for the calculation of total sleep time. Third, an actigraph was used to obtain the objective sleep index of total sleep time. An actigraph is an ecologically sound device that measures and stores movement information on a moment-tomoment basis. This device is worn as a wristwatch, referred to as an actiwatch, and is worn throughout the day and night across the 14 days.

Total sleep time was selected as the sleep variable of interest. This is because the study is testing the widely held assumption, particularly among laypersons, that total number of hours of sleep directly contributes to fatigue. It is important to note, however, that total wake time (TWT) is more commonly considered to be the most suitable indicator of disturbed sleep in the literature. TWT is a combination of sleep onset latency (i.e., the amount of time it takes to fall asleep) and wakefulness after sleep onset (i.e., the amount of time one is awake between during 
the middle of the night). Nevertheless, given that the current study was primarily interested in the amount of total sleep in relation to daytime fatigue, TST was used as the sleep index. This index was compared with sleep quality, a measure based largely on perception of how one slept the previous night.

Two different types of measurements were used to track fatigue over time. First, in the evening, participants were asked to record the degree to which they felt fatigued that day using a Visual Analogue Scale (VAS). Second, in addition to tracking sleep, the actiwatch also allows participants to press a button and enter a fatigue rating on a 10-point likert scale at three different points throughout the day; this served as the prospective assessment of daytime fatigue. The actiwatch fatigue ratings are recorded in the moment, whereas the VAS fatigue rating had the participant reflect over the past day; thus, each fatigue measurement coincides with the way in which sleep is measured on the actigraph and sleep diary respectively. The objective total sleep time index on the actigraph was used to predict the daily prospective fatigue ratings indicated on the actiwatch. The sleep quality ratings and total sleep time index on the sleep diary were used to predict fatigue on the VAS. Finally, retrospective, subjective self-report assessments of sleep and fatigue were also collected, in order to understand how participants perceive their sleep and fatigue. This compilation of measurement tools across multiple nights of assessment presents an important advantage of the current investigation, and overcomes some of the limitations discussed in the literature thus far. In addition to understanding how sleep and fatigue fluctuate alongside one another over time, this approach will help to overcome the problematic distinction between objective and subjective measurements of sleep and fatigue noted in the literature. This design can help further clarify whether daytime fatigue is directly related to objective or 
subjective indicators of poor sleep, or whether perhaps there is no temporal relationship between the two constructs.

The present study collected data from both individuals with and without insomnia for all analyses. Comparing those with insomnia to good sleepers may help us to understand whether and how the two groups differ in their experience of fatigue, which may help to further identify mechanisms that underlie the perpetuation of insomnia. Given the evidence suggesting that good and poor sleepers are more likely to differ on subjective indices of insomnia and distress, as opposed to objective sleep impairments (Fichten et al., 1995), it would be important to include both groups in studies such as this which make use of both subjective and objective indices of sleep impairment.

The secondary objective of this study is to explore factors other than poor sleep that may play a role in the experience of fatigue in insomnia. Indeed, as described above, theoretical models (e.g., Chaudhuri \& Behan, 2004; Harvey, 2002), as well as research studies have proposed that it could be other factors, such as cognitive-behavioural sequelae related to insomnia, which may help to explain the elevated fatigue found in insomnia sufferers (FortierBrochu et al., 2010; Ustinov et al., 2010). Such constructs include maladaptive beliefs about sleep, fear and avoidance of fatigue, and fatigue-based rumination. Each of these constructs was proposed as potential mediators of the relationship between sleep and fatigue. By understanding whether other factors, aside from poor sleep, may have a stronger impact on daytime fatigue, those with insomnia may alleviate some of the burden they place on their sleep, which would thus reduce sleep-related anxiety and help improve their overall functioning.

Ultimately, the main goal of this study is to understand the relationship between sleep and fatigue in people with insomnia. Given that daytime fatigue is among the most prevalent and 
distressing symptoms of insomnia, our appreciation for the experience of fatigue is vital for the prevention and treatment of this debilitating disorder. Understanding how fatigue is related to sleep is an important part of this process. Further, if it is found that non-sleep related factors play as or more of an important role in predicting fatigue, this may change how people with insomnia perceive and manage their illness.

\subsection{Hypotheses}

The primary objective of this study is to examine the relationship between sleep and fatigue among people with insomnia disorder (ID) and a normal sleeper (NS) comparison group. The following are the study objectives and hypotheses:

Objective 1: Investigate the nature of the temporal relationship between sleep quality (SQ) and fatigue across 14 days. In accordance with the literature suggesting that subjective indices of insomnia are strongly related to self-reported fatigue, the following hypothesis is proposed:

H1: Ratings of SQ on the sleep diary will be a significant predictor of fatigue ratings among ID and NS. Specifically, there would be a negative linear relationship between SQ and fatigue the following day, and this relationship would be consistent within- and between-persons. This hypothesis was examined in the sleep diary data only, as actigraphy does not allow for subjective rating of sleep quality.

Objective 2: Investigate the nature of the relationship between total sleep time (TST) and fatigue, using both subjective and objective measures of sleep. The following specific hypotheses are proposed:

H2a: TST on the sleep diary will be a significant predictor of VAS fatigue ratings, for both within- and between-person analyses. This hypothesis will apply to both ID and NS groups. 
$\mathrm{H} 2 \mathrm{~b}$ : Similar to the abovementioned hypothesis, TST on the actigraph will be a significant predictor of fatigue ratings on the actigraph across the two weeks. This is hypothesized to be the case for both ID and NS groups, as well as for both within- and between-person analyses.

Objective 3: Investigate group differences among ID and NS with respect to indices of sleep and fatigue. The following hypothesis is proposed:

H3: On the self-report measure of fatigue (i.e., the FSS), ID will report higher levels of fatigue compared to NS. This subjective relationship has been consistently demonstrated in the literature (Buysse et al., 2007; Hauri \& Fisher, 1986; Lichstein et al., 1997; Riedel \& Lichstein, 2000).

Objective 4: Investigate whether cognitive-behavioural factors contribute to our understanding of the relationship between sleep and fatigue. Thus, the final hypothesis is as follows:

H4: The relationship between self-reported sleep disturbance and self-reported fatigue will be mediated by cognitive-behavioural variables (i.e., maladaptive sleep beliefs, fear and avoidance of fatigue, and fatigue-based rumination). It is expected that these variables will mediate the relationship between sleep and fatigue in both ID and NS. 


\section{CHAPTER 3}

\section{METHOD}

\subsection{Participants}

Participants consisted of both people with insomnia disorder (ID) and a normal sleeper (NS) comparison group who were recruited from the community. Participants in the ID group met DSM-IV-TR ${ }^{1}$ and RDC criteria for an insomnia diagnosis based on the Duke Structured Interview for Sleep Disorders (DSISD). Specifically, individuals in the ID group reported at least one sleep complaint, including difficulty initiating sleep, difficulty maintaining sleep, or waking up too early, as well as one daytime complaint, such as fatigue, difficulty concentrating, or mood disturbances. Those in NS met research criteria for Normal Sleepers (Edinger et al., 2004), as verified by the DSISD. While sleep diary data were not available at the time of participant eligibility screening, those with ID who had sleep efficiencies over $85 \%$ were excluded from the study, while NS who scored below $85 \%$ on their sleep diaries were also excluded from the study. In addition, all participants were screened with the Structured Clinical Interview for DSM Axis I disorders (SCID-I), in order to assess for comorbid psychiatric disorders. Participants were excluded if they: 1) were not mentally competent to provide informed consent, 2) needed immediate psychiatric attention (e.g., imminently suicidal patients) or have attempted suicide in the past 6 months, 3) met criteria for highly sleep-disruptive disorders (i.e., Bipolar Disorder, Schizophrenia or any other psychotic disorders) on the basis of the SCID-I, 4) met criteria for Sleep Apnea, Restless Legs Syndrome, Circadian Rhythm Sleep Disorder, or excessive daytime sleepiness on the basis of the DSISD, or 5) had a history of alcohol, narcotic, benzodiazepine, or other substance abuse or dependence in the 6 months prior to screening. Participants in ID were

\footnotetext{
${ }^{1}$ DSM-5 criteria were not available at the time of the study.
} 
treatment-seekers, and they received four sessions of cognitive behavioural therapy for their insomnia. NS participants were compensated $\$ 40$ for their involvement in the study.

\subsection{Measures}

\subsubsection{Diagnostic Interviews}

The Duke Structured Interview for Sleep Disorders (DSISD: Edinger, Kirby, Lineberger, Loiselle, Wohlgemuth, \& Means, 2004). The DSISD is a structured interview designed to assess the presence of sleep disorders according to the DSM-IV-TR and ICSD-2 sleep disorder criteria. The interview is divided into four main modules, which respectively inquire about insomnia complaints, excessive daytime sleepiness, circadian rhythm sleep disorders, and parasomnias. In the present study, the DSISD was used to determine the presence or absence of insomnia, and to exclude participants who met criteria for any exclusionary sleep disorders. The DSISD has been shown to have good reliability (i.e., $r$ s range from 0.71 to 0.86 ) and is effective at distinguishing between different sleep disorders (Carney et al., 2009).

\section{Structured Clinical Interview for DSM-IV-TR Axis I Disorders (SCID-I: First,} Spitzer, Gibbon, \& Williams, 2002). The SCID-I is a semi-structured interview used for diagnosing Axis I disorders. In the present study, it was used to assess for Axis I comorbid disorders and to exclude participants who met criteria for any exclusionary disorders (i.e., Bipolar Disorder, psychosis, or substance-use). Master's and $\mathrm{PhD}$ students administered the SCID-I and followed the standard administration and scoring procedures. Studies have documented fair to excellent kappa values for both test-retest and inter-rater reliability for nearly all Axis I Disorders on the SCID-I. For example, the inter-rater reliability of 12 DSM-IV diagnoses ranged from 0.61 to 0.83 , with a mean kappa of 0.71 (Lobbestael, Leurgans, \& Arntz, 
2011). Another study reported kappas ranging from 0.57 to 1.0 and 0.35 to 0.78 for inter-rater and test-retest reliability, respectively (Zanarini et al., 2000).

\subsubsection{Outcome Measures}

Sleep Diary (Carney et al., 2012) (See Appendix A). Sleep diaries are the recommended tool for the assessment of insomnia symptoms (Buysse et al., 2006). A sleep diary is a daily log which inquires about the previous night's sleep. In particular, it asks participants to report on information such as the length of time it takes to fall asleep, number and length of nocturnal awakenings, time of final awaking, and rising time. In addition, the diary requests participant ratings of the quality of each night's sleep and how rested they felt upon wakening. Their sleep quality rating is made on a 5-point likert scale ranging from "very good" to "very poor". The information derived from these sleep logs comprise several sleep indices, which include sleep onset latency (SOL), time spent awake after initially falling asleep or "wakefulness after sleep onset" (WASO), total sleep time (TST), time in bed (TIB) and the percentage of time in bed spent sleeping or "sleep efficiency" (SE). The above are common subjective indices recommended in the sleep diary literature (Buysse et al., 2006). In the present study, TST was used as the index of sleep. Sleep Quality ratings were also used in the present study. The instructions define sleep quality as, "your sense of whether your sleep was good or poor" (Carney et al., 2012, p.294).

The sleep diary has been used for decades in insomnia research and is considered to be a reliable and valid assessment of insomnia (Coates et al., 1981; Moul, Hall, Pilkonis, \& Buysse, 2004; Smith \& Trinder, 2001; Spielman, Saskin, \& Thorpy, 1987). The present study used the Consensus Sleep Diary, which is the recently published standardized sleep diary proposed for use in a wide range of populations (Carney et al., 2012). All information was recorded daily 
upon wakening over a two-week monitoring period of the study. A 14-day interval is necessary in order to achieve adequate stability (i.e., $G$ coefficient of at least .80 ) for many of the variables of interest recorded in the home setting, including TST (Wohlgemuth, Edinger, Fins, \& Sullivan, 1999). By assessing sleep across 14 days, the sleep diary is considered to be the best subjective tool to capture the night-to-night variability of sleep (Buysse et al., 2006).

Visual Analog Scale (VAS) (See Appendix B). The VAS consists of a single item querying the degree to which participants are fatigued or energized. Participants were instructed to mark an " $\mathrm{X}$ " on a 100 millimetre line which represents the degree to which they felt fatigued/energized over the course of the day. The VAS is a bipolar scale, whereby the responses range from "extremely fatigued" to "extremely energized". The VAS was scored with a ruler, whereby the millimeter distance from the left edge of the line to the centre of the $\mathrm{X}$ is the score given for the item. In the present study, participants completed the VAS at the end of the day, and report on their levels of fatigue/energy throughout the course of the day. Thus, it is a retrospective measure, which was used as the outcome variable for subjective TST and Sleep Quality on the sleep diary. Using a bipolar VAS to assess for fatigue is optimal for testing those with and without insomnia, as good sleepers may report higher energy levels as opposed to fatigue. In this sense, the VAS allows for more variability in responses (i.e., it is difficult to remember the exact spatial location of the last rating), and will manage restriction of range or demand characteristic problems. In addition, using a daily rating of fatigue on a VAS as opposed to a questionnaire coincides with the daily fatigue ratings being collected on the actiwatch.

Actigraphy: Mini-Mitter ${ }^{\mathrm{R}}$ actigraphs were used to derive objective indicators of sleep. The actigraph is a portable device worn like a wristwatch which uses an accelerometer to measure physical movement during wake and sleep (American Sleep Disorders Association, 
1997; Ancoli-Israel et al., 2003; Buysse et al., 2006; Hauri \& Wisbey, 1992; Sadeh \& Acebo, 2002). Using a validated algorithm of characterizing wakeful versus rest movements, this measure provides an objective evaluation of participants' sleep on a moment-to-moment basis, and, like the sleep diary, reports on indices of sleep including TST, TIB, SOL, WASO and SE. The epoch length for the current study was set to 1 minute, indicating that the actiwatch would sample the degree of activity/rest once every minute. In addition, the actiwatch sounds an alarm at three different time points (10:00am, 1:00pm and 7:00pm) each day, prompting participants to provide a fatigue rating on a scale from 0 to 10 . This momentary rating served as a prospective measure of fatigue. The mean of the three daily ratings were used as the daily fatigue measure, as we know that levels of fatigue vary throughout the day. For example, fatigue is typically high in the hour after waking due to sleep inertia (Tassi \& Muzet, 2000) and is also higher in the hour after lunch due to the post-lunch dip in body temperature (Hayashi, Watanabe, \& Hori, 1999); these are referred to as "time of day" effects. Thus, taking the mean of three measures throughout the day will control for these time of day effects by ensuring that participants are not routinely completing their fatigue ratings during a time of day when their fatigue is likely to be higher or lower than normal. In the present study, the actiwatch was used to obtain an objective index of TST, which was used to predict the mean daytime fatigue ratings across the two weeks.

A one-minute epoch length was used for the current study. Actiwatch software scores each epoch as either sleep or wake as determined by the number of activity counts per epoch (i.e., per minute), as well as the activity counts for the epochs immediately surrounding the given epoch. Specifically, the activity counts for the two epochs immediately before and after the epoch are multiplied by $1 / 5$ and the two epochs beyond that are multiplied by $1 / 25$. These values are then added to the activity count for the epoch in question, which is then compared to a 
sensitivity threshold value for wakefulness. For the present study, the sensitivity threshold was set to 20 (Lichstein et al., 2006); epochs above this value were scored as wake whereas activity counts below this value were scored as sleep. Total sleep time was calculated by the total number of minutes of "sleep" according to the Actiwatch software.

There is widespread empirical support for the use of actigraphy in the assessment of sleep variables (Ancoli-Israel et al., 2003; Sadeh \& Acebo, 2002). Specifically, actiwatch data has been found to correlate with PSG measures in the assessment of TST, SOL, WASO, and SE (Edinger, Means, Stechuchak, Olsen, 2004). Given its portable nature and inexpensive cost, actigraphy, like sleep logs, has been used to assess night-to-night variability in sleep. In particular, studies have suggested that 14 days of actiwatch data is recommended to determine variability in sleep (Rowe et al., 2008; van Hilten et al., 1993). Thus, in the present study, participants wore the actiwatch for a two-week period. A one-minute epoch length was used in order to gather data across the two weeks.

\subsubsection{Self-Report Questionnaires}

The Insomnia Severity Index (ISI: Morin, 1993). The ISI is a recommended self-report measure for assessing insomnia (Buysse et al., 2006). It is a 7-item scale which measures the severity of insomnia symptoms as well as degree of dissatisfaction, daytime interference, noticeability of impairment, and distress caused by the sleep disturbance. Each item is measured on a 5-point Likert scale ranging from 0 ("not at all") to 4 ("extremely"). Total scores range from 0 to 28 , with higher scores suggesting increased insomnia severity. The recommended interpretation guidelines are as follows: scores of $0-7$ suggest no clinical insomnia, scores of 8

- 14 suggest sub-threshold insomnia, scores of $15-21$ suggest moderate insomnia, and scores of $22-28$ suggest severe insomnia (Bastien, Vallières, \& Morin, 2001). The ISI has been found to 
have very good internal consistency (Cronbach's alpha $=.91$ ) (Morin, Belleville, Belanger, \& Ivers, 2011; Sierra, Guillén-Serrano, \& Santos-Iglesias, 2008) and good convergent validity, as it correlates with sleep diary measures and polysomnography (Bastien et al., 2001), as well as measures of fatigue, anxiety and depression (Morin et al., 2011). In the present study, the ISI was used as the self-report measure of insomnia to test the mediation model (i.e., whether cognitivebehavioural variables mediate the relationship between insomnia and fatigue).

Fatigue Severity Scale (FSS: Krupp et al., 1989). The FSS is a frequently used 9-item scale which measures fatigue symptoms in several populations. Items are scored along a 7-point Likert scale ranging from 1 ("strongly agree") to 7 ("strongly disagree"). The FSS total score is the average of the 9 individual items, with higher scores indicating higher degrees of fatigue. The scale has good psychometric properties, as demonstrated by excellent internal consistency $($ Cronbach's alpha $=.94)$ and good test-retest reliability (Krupp et al., 1989; Valko et al., 2008). The FSS has also been found to be correlated with VAS measures of fatigue (Krupp et al., 1989), demonstrating good convergent validity. While this scale has primarily been validated in medical disorder populations (Armutlu et al., 2007; Krupp et al., 1989), more recent research has explored its validity in sleep disorder samples. Specifically, in a recent validation study, the FSS was found to distinguish the severity of fatigue between insomnia and healthy populations (Valko et al., 2008). While fatigue scores are higher on the FSS in insomnia populations compared to good sleepers, there is still a moderate degree of fatigue and a considerable range of FSS scores found in those without insomnia (i.e., Ms range from 2.3 to 3.4; SDs range from 0.7 to 1.2; Krupp et al., 1989; 1993; Ferentinos et al., 2011; Lichstein et al., 1997; Means et al., 2000; Valko et al., 2008). Thus restriction of range in the FSS among NS is not likely to be problematic. Although other measures of fatigue (e.g., MFI) have been validated for use in 
insomnia, the FSS is briefer and thus reduces participant burden. In the present study, the FSS was used as the self-report measure of fatigue to test the mediation model (i.e., whether cognitive-behavioural variables mediate the relationship between insomnia and fatigue). It was also used to determine whether ID and NS differ on subjective fatigue.

The Dysfunctional Beliefs and Attitudes about Sleep Scale (DBAS-16: Morin et al., 2007). The DBAS-16 is a self-report measure assessing unhelpful sleep-related beliefs. The DBAS-16 is an abbreviated scale derived from the original 30 -item DBAS. The measure assesses beliefs about the consequences of insomnia, worry about sleep, sleep expectations, and causal attributions for insomnia. The individual rates his/her level of agreement with each statement on a 10-point scale ranging from 0 ("strongly disagree") to 10 ("strongly agree"). The total score is obtained via a mean item score, with higher mean scores representing more maladaptive sleep beliefs. The DBAS-16 demonstrates adequate internal consistency (Cronbach's alpha $=.79$ ) and has appropriate convergent validity with the ISI, sleep diaries and polysomnography (Morin et al., 2007). It can also effectively discriminate between those who do and do not have clinical levels of unhelpful sleep beliefs via a cutoff score of 3.8 , which maximized both sensitivity (80\%) and specificity (76\%) based on an ROC curve (Carney et al., 2010). In the present study, the DBAS will be used as the measure of maladaptive sleep beliefs, and will be tested as a potential mediator of the relationship between insomnia and fatigue.

The Fear of Fatigue Scale (FOF: Harris \& Carney, 2009) (See Appendix C). The FOF measures concern about experiencing fatigue. This scale was adapted from The Fear Inventory (FI: Taylor \& Rachman, 1992) [which was modified from the Fear Survey Schedule (FSS-III: Wolpe \& Lang, 1964)] and was developed for the purposes of this study. The FOF contains 12 items which ask participants the extent to which they are afraid of experiencing fatigue. 
Examples of items include fears of: "getting up early in the morning", "looking so sleepy people might notice", and "feeling moderately tired", which were generated based on clinical experience. Participants indicate their level of fear of each item on a 5-point scale ranging from 0 ("not at all") to 4 ("very much afraid"). Research on the FOF has suggested that relative to good sleepers, self-reported poor sleepers report increased fear/aversion of the experience of fatigue symptoms (Harris \& Carney, 2009). In the present study, the FOF was used as the measure of fear of fatigue, and was tested as a potential mediator of the relationship between insomnia and fatigue.

The Avoidance of Fatigue Scale (AOF: Harris \& Carney, 2009) (See Appendix C). The AOF measures avoidance of fatigue-related stimuli. The scale was modified from the original 26-item Mobility Inventory (Chambless, Caputo, Jasin, Gracely, \& Williams, 1985). The current version of the AOF contains the same 12 items that make up the FOF, including items measuring feeling tired, sleepy, and foggy-headed. The instructions ask participants the extent to which they avoid feeling/looking fatigued on a 5-point scale ranging from 0 ("not at all") to 4 ("very much"). Preliminary research on the AOF suggests that relative to good sleepers, poor sleepers report an increased desire to avoid the experience of fatigue (Harris \& Carney, 2009). The AOF was used as the measure of avoidance of fatigue, and was tested as a potential mediator of the relationship between insomnia and fatigue.

Daytime Insomnia Symptom Response Scale (DISRS; Carney, Harris, Falco \& Edinger, 2013) (See Appendix D). The DISRS is a 20-item scale, which is an expanded version of the 8-item Symptom-Focused Rumination subscale of the Responses Styles Questionnaire (RSQ: Nolen-Hoeksema, 1991). Twelve additional items based on daytime symptoms of insomnia were generated by Dr. Colleen Carney based on clinical expertise. Sample items 
include, "Think about how everything requires more effort than usual", "Think about how tired you feel", and "Think about how you don't have the energy to get through the day". Participants are asked how frequently they endorse these 20 items when they are feeling down or tired, on a 4-point scale ranging from 1 (“almost never") to 4 (“almost always"). Total scores range from 20 to 80 , with higher scores indicating higher levels of fatigue-based rumination. In a previous investigation, the internal consistency, as measured by Cronbach's alpha, was .93 in an undergraduate sample (Harris, Carney, \& Moss, 2010). Cronbach's alpha in a clinical sample was .94 and the DISRS was found to be correlated with theoretically relevant constructs, including insomnia, fatigue and mood disturbance (Carney et al., 2013). In the present study, the DISRS will be used as the measure of fatigue-based rumination, and will be tested as a potential mediator of the relationship between insomnia and fatigue.

The Depression Anxiety Stress Scales (DASS-21: Lovibond \& Lovibond, 1995). The DASS-21 is an abbreviated measure derived from the original 42 -item scale. It inquires about the degree to which participants feel 1) depressed, 2) anxious or 3) stressed, over the past week; each of these constructs forms a distinct subscale. The Depression scale assesses for symptoms associated with low mood (e.g., sadness or hopelessness). The Anxiety scale primarily assesses for physiological arousal, such as symptoms associated with panic attacks (e.g., trembling and racing heart). Finally, the Stress scale inquires about symptoms related to tension, difficulty relaxing, and irritability (Antony, Bieling, Cox, Enns, \& Swinson, 1998). The individual rates the applicability of each statement on a 4-point scale ranging from 0 (“did not apply to me at all") to 3 ("applied very much to me or most of the time"). Subscale scores are added together and then multiplied by two, with total subscale scores ranging from 0 to 42 . Each subscale selects different cutoff scores to determine whether the degree of distress is mild, 
moderate, severe, or extremely severe (see Lovibond \& Lovibond, 1995). The internal consistency of each of the Depression, Anxiety and Stress scales are high (Cronbach's alphas are $.94, .87$ and .91 respectively). With respect to convergent validity, the DASS-21 demonstrated moderate to high correlations with other self-report measures of depression and anxiety (Antony et al., 1998). In the present study, both the Anxiety and Stress scales will be controlled for in the mediation analyses with the DBAS-16, FOF and AOF. The Depression scale will be controlled for in the DISRS mediation analysis.

\subsection{Procedure}

This study comprised two visits to the Sleep and Depression Laboratory at Ryerson University, as well as two weeks of sleep and fatigue monitoring at home. First, participants called the laboratory if, after reading posted study advertisements, they were interested in the study. Participants were recruited from Craigslist, the Metro, and flyers which were posted in and around Ryerson University campus as well as the surrounding downtown Toronto area. As we have had several clinical trials ongoing in our lab, we did not need to advertise specifically for poor sleepers for this study. We have had a steady flow of poor sleepers interested in our lab's clinical trials who have found out about the study either through online or printed advertisements, physician referrals, and word of mouth. Once they called the lab expressing their interest, if they met eligibility criteria, they were included in the study.

If participants met initial eligibility criteria (i.e. for either the ID or NS group) based on the phone screen, they were invited in for a screening appointment. In the initial visit, the study coordinator reviewed the informed consent form with participants and allowed time for any questions they might have. At this time, the study candidates were informed about the purpose and description of the study as well as the risks and benefits associated with participation. 
Participants provided verbal and written consent in order to proceed with the study. Once consent was obtained, the Masters- and PhD-level study staff administered the DSISD and SCID-I in order to determine whether participants met eligibility criteria for either insomnia disorder (ID) or normal sleeper (NS).

Once participants were deemed eligible for the study, they were asked to complete a packet of questionnaires, which included the ISI, FSS, DBAS, DISRS, FOF and AOF. Next, participants were trained on how to complete the sleep and fatigue monitoring over the subsequent two weeks. Specifically, participants were expected to monitor: 1) their subjective sleep and fatigue on the sleep diary and VAS for two weeks, and 2) their objective sleep and three daytime, prospective measures of fatigue for two weeks using the actiwatch. The sleep diary was filled out every morning for two weeks with reference to the previous night's sleep. This log included a bipolar VAS assessing degree of fatigue/energy, which was completed in the evening. The actiwatch monitored activity and movement at a rate of one minute during the day and at night. In addition, the actiwatch sounded an alarm at 10:00am, 1:00pm and 7:00pm each day. When the alarm sounded, participants were asked to enter a fatigue rating on a scale from 0 to 10 , to indicate their level of fatigue at that moment.

After completing the two-weeks of monitoring, participants came back to the lab to return their sleep logs and the actiwatch. At this time, NS were compensated for their time and the ID participants received treatment for their insomnia, as a second part to this study which will not be described here.

\subsection{Statistical Analyses}

The primary study hypotheses were tested with hierarchical linear modeling (HLM) (Bryk \& Raudenbush, 1992), which is an extension of the general linear model. HLM does not 
require observations to be independent and instead caters to the hierarchical structure of the data. That is, it tests daily observations nested within each individual, which enables us to perform the hierarchical analyses needed for this data. For the purposes of the present study, in order to examine the intra-individual variability of sleep and fatigue, separate analyses were conducted for objective and subjective measures of sleep. One analysis tested whether: a) subjective sleep quality ratings (i.e., Hypothesis 1), and b) total sleep time on the sleep diary (i.e., Hypothesis 2a) predicted daytime VAS ratings of fatigue over the course of two weeks. A second analysis tested whether objective sleep (i.e., TST) predicted the mean of the three prospective daytime fatigue ratings on the actiwatch over the course of two weeks (i.e., Hypothesis $2 b$ ). Thus, these analyses test whether nighttime sleep has an effect on daytime fatigue, both between- and withinindividuals, over time. A one-way ANOVA was used to test whether fatigue scores on the FSS are higher among those with ID compared to NS (i.e., Hypothesis 3).

A bootstrapping mediation analysis was used to test the mediator model (Hayes, 2009). Four simple mediation analyses tested whether each of the cognitive-behavioural variables mediate the relationship between sleep and fatigue among ID and NS (i.e., Hypothesis 4). The Anxiety and Stress subscales of the DASS were entered as covariates in the DBAS, FOF, and AOF analyses, whereas the Depression subscale acted as the covariate for the DISRS mediation analysis. As previously stated, this is because anxiety is the mood state most associated with fear, avoidance, and maladaptive beliefs, whereas depression is the mood state most commonly found in rumination. In these analyses, the ISI and FSS were used as the measures of self-reported insomnia and fatigue, respectively, in order to reflect the same measurement approach (i.e., retrospective self-report) as is found in the proposed mediators (i.e., DBAS, FOF, AOF, and DISRS). The indirect effect of these variables were tested using a confidence interval of 95 and 
was resampled 10,000 times. Bootstrapping has been shown to be one of the most valid and powerful tools in testing mediation (MacKinnon, Lockwood, \& Williams, 2004; Williams \& MacKinnon, 2009). 


\section{CHAPTER 4}

\section{RESULTS}

\subsection{Participant Demographic Characteristics}

A total of 46 individuals with ID and 48 NS were eligible to participate in the study. Six participants in the insomnia group dropped out before beginning to collect data, three participants did not records any VAS ratings, two participants had missing data on their sleep logs which did not allow for calculations of TST, one had substantial missing data on the selfreport measures, and two had sleep efficiencies that were higher than what would be expected for normal sleepers (i.e., above $85 \%$ sleep efficiency). Three participants in the normal sleeper group dropped out, five participants did not return their data packages or only returned half of the package (i.e., either the sleep or fatigue component), four had missing data on their sleep logs which did not allow for calculations of TST, two had values suggestive of a Circadian Rhythm Disorder (e.g., reports of sleeping during the day more often than during the night), and two participants had illnesses while completing the sleep and fatigue ratings. No significant differences were found between study completers and participants who dropped out, as well as those who had missing/unusable data, on self-reported fatigue $[F(2,87)=0.91, p=.41]$ or selfreported insomnia $[F(2,86)=1.09, p=.34]$. The final sample consisted of 33 participants with ID and 32 NS (total $N=65$ ). Participant demographic characteristics are presented in Table 1. Those with ID $(M=49.64, S D=12.86)$ were significantly older than NS $(M=32.36, S D=$ 14.27), $F(1,64)=26.67, p<.001$, and therefore age was entered as a covariate in subsequent analyses which separated NS from ID. The groups did not differ significantly on any other demographic characteristics. 
Table 1

Participant Demographics for Normal Sleepers, Insomnia Disorder, and the Total Sample

\begin{tabular}{|c|c|c|c|c|}
\hline & $\begin{array}{l}\text { Total Sample } \\
\qquad(\mathrm{N}=65)\end{array}$ & $\begin{array}{c}\mathrm{NS} \\
(n=32)\end{array}$ & $\begin{array}{c}\mathrm{ID} \\
(n=33)\end{array}$ & \\
\hline & $n$ & $n$ & $n$ & $\chi^{2}$ \\
\hline \multicolumn{5}{|l|}{ Sex } \\
\hline Female & 53 & 24 & 29 & \\
\hline Male & 12 & 8 & 4 & 1.79 \\
\hline \multicolumn{5}{|l|}{ Ethnicity } \\
\hline European Canadian & 44 & 19 & 25 & \\
\hline East/Southeast & 10 & 7 & 3 & \\
\hline \multicolumn{5}{|l|}{ Asian Canadian } \\
\hline African Canadian & 2 & 2 & 0 & \\
\hline West Asian/Arab & 2 & 0 & 2 & \\
\hline \multicolumn{5}{|l|}{ Canadian } \\
\hline Latin/Central/South & 2 & 1 & 1 & \\
\hline \multicolumn{5}{|l|}{ Canadian } \\
\hline Caribbean Canadian & 1 & 0 & 1 & \\
\hline Other/Multiple & 4 & 3 & 1 & 11.04 \\
\hline \multicolumn{5}{|l|}{ Employment Status } \\
\hline Full-time & 31 & 14 & 17 & \\
\hline Part-time & 19 & 12 & 7 & \\
\hline Not working & 15 & 6 & 9 & 2.19 \\
\hline
\end{tabular}

Note. $\mathrm{NS}=$ Normal sleepers, ID = Insomnia disorder 
Prior to conducting the primary analyses, the self-report, sleep diary, and actigraphy data were screened for violations of normality. An inspection of the distribution of the mean scores on the self-report measures (i.e., ISI, FSS, DBAS, DISRS, FOF, and AOF) suggest that the distributions approximated normal, as the skewness and kurtosis values were within the acceptable ranges of $|2|$ and $|7|$ respectively (West, Finch, \& Curran, 1995). Total sleep time on the sleep diary (TST-SD), sleep quality on the sleep diary (SQ-SD) and fatigue ratings on the VAS (FR-VAS), as well as TST on the actigraph (TST-A) and fatigue ratings on the actigraph (FR-A), were also found to be normally distributed.

The means, standard deviations, and Cronbach's alphas for each of the self-report measures among ID, NS, and the total sample are displayed in Table 2. On each of the measures, NS scored significantly lower than ID after controlling for age; therefore, the psychometrics of these measures in the current sample were deemed to be acceptable and comparable to previous studies. The correlations among these self-report measures are presented in Table 3. All of the questionnaires were significantly correlated with one another $(p<.001)$. The means of the sleep diary and actigraphy sleep and fatigue indices are reported in Table 4 . Each of the indices differed significantly among NS and ID. The correlations among the sleep and fatigue indices on both the sleep diary and the actigraph are presented in Table 5 . 
Table 2

Means, Standard Deviations, and Cronbach's Alphas for Self-Report Measures

\begin{tabular}{|c|c|c|c|c|c|c|c|c|}
\hline \multirow{2}{*}{$\begin{array}{l}\text { Self- } \\
\text { Report } \\
\text { Measures }\end{array}$} & \multicolumn{3}{|c|}{$\begin{array}{l}\text { Total Sample } \\
\qquad(N=65)\end{array}$} & \multicolumn{2}{|c|}{$\begin{array}{c}\mathrm{NS} \\
(n=32)\end{array}$} & \multicolumn{2}{|c|}{$\begin{array}{c}\text { ID } \\
(n=33)\end{array}$} & \multirow[b]{2}{*}{${ }^{\mathrm{a}} F$} \\
\hline & $M$ & $S D$ & Alpha & $M$ & $S D$ & $M$ & $S D$ & \\
\hline ISI & 10.71 & 9.66 & 0.97 & 1.56 & 1.74 & 19.86 & 3.71 & $464.73 * *$ \\
\hline FSS & 3.41 & 1.58 & 0.94 & 2.21 & 0.90 & 4.59 & 1.17 & $69.07 * *$ \\
\hline DBAS & 4.16 & 2.33 & 0.93 & 2.27 & 1.29 & 5.93 & 1.56 & $79.37 * *$ \\
\hline DISRS & 37.12 & 13.69 & 0.96 & 29.34 & 9.53 & 44.67 & 12.94 & $27.00 * *$ \\
\hline FOF & 12.14 & 11.00 & 0.90 & 6.00 & 6.83 & 18.09 & 11.08 & $19.84 * *$ \\
\hline $\mathrm{AOF}$ & 15.64 & 11.42 & 0.90 & 11.00 & 10.94 & 20.29 & 10.05 & $14.66 * *$ \\
\hline
\end{tabular}

Note. NS = Normal sleepers, ID = Insomnia disorder, ISI = Insomnia Severity Index, FSS = Fatigue Severity Scale, DBAS = Dysfunctional Beliefs and Attitudes about Sleep Scale, DISRS $=$ Daytime Insomnia Symptom Response Scale, FOF $=$ Fear of Fatigue Scale, AOF = Avoidance of Fatigue Scale.

${ }^{\text {a }}$ Group difference analyses controlled for age

$* * p<.001$ 
Table 3

Correlations among Self-Report Measures for the Total Sample

\begin{tabular}{lcccccc}
\hline Measure & 1 & 2 & 3 & 4 & 5 & 6 \\
\hline 1. ISI & -- & $.86^{* *}$ & $.85^{* *}$ & $.67^{* *}$ & $.63^{* *}$ & $.42^{* *}$ \\
2. FSS & -- & $.87^{* *}$ & $.81^{* *}$ & $.65^{* *}$ & $.47^{* *}$ \\
3. DBAS & & -- & $.75^{* *}$ & $.72^{* *}$ & $.56^{* *}$ \\
4. DISRS & & & -- & $.64^{* *}$ & $.50^{* *}$ \\
5. FOF & & & & -- & $.73^{* *}$ \\
6. AOF & & & & & -- \\
\hline
\end{tabular}

Note. ISI = Insomnia Severity Index, FSS $=$ Fatigue Severity Scale, DBAS $=$ Dysfunctional Beliefs and Attitudes about Sleep Scale, DISRS = Daytime Insomnia Symptom Response Scale, $\mathrm{FOF}=$ Fear of Fatigue Scale, AOF $=$ Avoidance of Fatigue Scale.

$* * p<.001$ 
Table 4

Means and Standard Deviations Scores on Sleep Diary and Actigraphy Indices

\begin{tabular}{|c|c|c|c|c|c|c|c|}
\hline \multirow[b]{2}{*}{ Sleep Diary } & \multicolumn{2}{|c|}{$\begin{array}{l}\text { Total Sample } \\
\quad(N=65)\end{array}$} & \multicolumn{2}{|c|}{$\begin{array}{c}\mathrm{NS} \\
(n=32)\end{array}$} & \multicolumn{2}{|c|}{$\begin{array}{c}\text { ID } \\
(n=33)\end{array}$} & \multirow[b]{2}{*}{$F$} \\
\hline & $M$ & $S D$ & $M$ & $S D$ & $M$ & $S D$ & \\
\hline TST & 6.64 & 1.71 & 7.45 & 1.36 & 5.83 & 1.65 & $128.80 * *$ \\
\hline SQ & 3.32 & 1.13 & 4.01 & 0.75 & 2.64 & 1.03 & $391.70 * *$ \\
\hline SOL & 0.54 & 0.74 & 0.27 & 0.40 & 0.81 & 0.89 & $121.09 * *$ \\
\hline WASO & 0.59 & 1.23 & 0.37 & 1.47 & 0.81 & 0.88 & $22.73 * *$ \\
\hline $\mathrm{SE}$ & $82 \%$ & 0.17 & $92 \%$ & 0.07 & $72 \%$ & 0.17 & $364.75^{* *}$ \\
\hline FR-VAS & 5.46 & 2.55 & 6.80 & 2.10 & 4.14 & 2.26 & $309.30 * *$ \\
\hline Actigraphy & \multicolumn{2}{|c|}{$N=43$} & \multicolumn{2}{|c|}{$n=23$} & \multicolumn{2}{|c|}{$n=20$} & \\
\hline TST & 5.21 & 1.71 & 5.58 & 0.93 & 4.66 & 1.33 & $34.75 * *$ \\
\hline SOL & 0.36 & 0.59 & 0.41 & 0.68 & 0.30 & 0.45 & 1.58 \\
\hline WASO & 2.42 & 1.71 & 2.02 & 1.51 & 2.93 & 1.80 & $42.63 * *$ \\
\hline $\mathrm{SE}$ & $63 \%$ & 19.0 & $68 \%$ & 16.05 & $58 \%$ & 20.8 & $41.85 * *$ \\
\hline FR-A & 3.31 & 2.36 & 2.11 & 1.23 & 4.74 & 2.00 & $226.04 * *$ \\
\hline
\end{tabular}

Note. NS = Normal sleepers, ID = Insomnia disorder, TST $=$ Total sleep time, $\mathrm{SQ}=$ Sleep quality, $\mathrm{SOL}=$ Sleep onset latency, WASO $=$ Wakefulness after sleep onset, $\mathrm{SE}=$ Sleep efficiency, FR-VAS = Fatigue ratings on the visual analogue scale, FR-A = Fatigue ratings on the actigraph.

$* * p<.001$ 
Table 5

Correlations among Sleep and Fatigue Indices on the Sleep Diary and Actigraphy for the Total Sample

\begin{tabular}{|c|c|c|c|c|c|c|c|c|c|c|c|}
\hline Measure & 1 & 2 & 3 & 4 & 5 & 6 & 7 & 8 & 9 & 10 & 11 \\
\hline 1.TST-SD & -- & $.50 * *$ & $-.39 * *$ & $-.11 * *$ & $.72 * *$ & $.42 * *$ & $.30 * *$ & -.03 & -.04 & $.17 * *$ & $-.35^{* *}$ \\
\hline 2. SQ-SD & & -- & $-.40 * *$ & $-.13 * *$ & $.56^{* *}$ & $.62 * *$ & $.31 * *$ & -.03 & $-.26 * *$ & $-.30 * *$ & $-.50 * *$ \\
\hline 3. SOL-SD & & & -- & .06 & $-.66^{* *}$ & $-.32 * *$ & .01 & $.17^{* *}$ & $.09^{*}$ & $-.10 *$ & $.40 * *$ \\
\hline 4. WASO-SD & & & & -- & $-.30 * *$ & $-.11 * *$ & $-.20 * *$ & -.03 & $.34 * *$ & $-.29 * *$ & $.32 * *$ \\
\hline 5. SE-SD & & & & & -- & $.45^{* *}$ & $.14 * *$ & -.03 & $-.22 * *$ & $.22 * *$ & $-.43 * *$ \\
\hline 6. FR-VAS & & & & & & -- & $.22 * *$ & -.03 & $-.16 * *$ & $-.16 * *$ & $-.59 * *$ \\
\hline 7. TST-A & & & & & & & -- & .02 & $-.66 * *$ & $.85^{* *}$ & $-.21 * *$ \\
\hline 8. SOL-A & & & & & & & & -- & $-.12 * *$ & $-.12 * *$ & .07 \\
\hline 9. WASO-A & & & & & & & & & -- & $-.87 * *$ & $.21 * *$ \\
\hline 10. SE-A & & & & & & & & & & -- & $-.26 * *$ \\
\hline 11. FR-A & & & & & & & & & & & -- \\
\hline
\end{tabular}

Note. TST-SD = Total sleep time on the sleep diary, SQ-SD = Sleep quality on the sleep diary, SOL-SD = Sleep onset latency on the sleep diary, WASO-SD = Wakefulness after sleep onset on the sleep diary, SE-SD = Sleep efficiency on the sleep diary, FR-VAS $=$ Fatigue ratings on the visual analogue scale, TST-A = Total sleep time on the actigraph, SOL-A = Sleep onset latency on the actigraph, WASO-SD = Wakefulness after sleep onset on the actigraph, SE-A = Sleep efficiency on the actigraph ,FR-A = Fatigue ratings on the actigraph.

$* * p<.001 * p<.01$ 


\subsection{Hierarchical Linear Modeling}

In order to address Hypotheses 1 through 3, hierarchical linear modeling (HLM) (Bryk \& Raudenbush, 1992) was used to examine whether nighttime sleep predicts daytime fatigue levels among ID and NS, across two weeks. HLM is ideally suited to perform these analyses as it uses nested data structures and does not require that observations within persons be independent (Raudenbush \& Bryk, 2002). Thus, in the current study, 14 days of observations (i.e., sleep and fatigue indices) are nested within 65 individual participants, thereby providing a nested data structure. Given that individuals differ systematically from one another (e.g., some individuals consistently sleep less compared to others), dependence of observations (i.e., observations for one individual cluster more closely together than for another individual) is expected in nested data structures. Unlike traditional data analytic techniques, HLM does not require that observations be independent within persons, which is an important advantage of this technique.

Another key advantage of HLM is that it is robust against missing data, which is particularly important when participants are asked to provide several data points per day across two weeks. More specifically, HLM uses maximum likelihood estimation, which can analyze the data as if no data points were missing (Raudenbush \& Bryk, 2002). The programs which use maximum likelihood estimation, such as the PROC MIXED program in SAS used in the current study, view the unknown, or missing data as a source of random variation which are removed from the likelihood by a process of integration. Instead of imputing new values, as more traditional methods might use, this program removes the values from the inferential system by a processing of averaging over the values (Collins, Shafer \& Kam, 2001). Maximum likelihood estimation operates under the assumption that the data missing at random. Data missing at random suggests that there is no relationship between missingness and the outcome variable of 
interest (Collins et al., 2001; Rubin, 1976). In the present study, this assumption was satisfied, as there is no reason to believe the missing data had a significant relationship with the sleep or fatigue indices under investigation.

A final benefit of HLM is its allowance for the examination of both within- (level 1) and between- (level 2) person variables. As discussed in the introduction, between-person variables have often been the primary focus in the sleep literature; therefore, the ability to analyze withinperson sleep variables was an important additional endeavor of the present study. Level 1 models determine whether an individual who obtains fewer hours of sleep on one night relative to their average nightly total sleep time leads to increased fatigue the following day (i.e., withinpersons). Level 2 models, on the other hand, ask whether people who generally report less total sleep time have higher levels of fatigue during the day (i.e., between-persons). The distinction between level 1 and level 2 analyses will be further clarified in the subsequent section.

The MIXED Procedure in SAS version 9.3 was used to perform the HLM using Restricted Maximum Likelihood (REML) estimation. In the current investigation, two separate models were used to predict daytime fatigue. In the first model, SQ-SD and TST-SD were the predictor variables of interest and FR-VAS was the dependent variable (i.e., addressing Hypotheses 1 and 2a). In the second model, TST-A was used to predict the average of three momentary daytime assessments of fatigue as indicated on the actiwatch (i.e., FR-A; addressing Hypothesis 2 b). As mentioned above, sleep quality ratings will only be used in the sleep diary analyses, as there is no available subjective sleep quality rating equivalent on the actigraph. Thus, the investigation of these hypotheses were separated into Sleep Diary and Actigraphy sections. 
Prior to evaluating these hypotheses, the error structure of the data required examination. When collecting repeated measures data, observations closer together in time are more highly correlated with one another than observations that are further apart. In order to account for this potential bias, an autoregressive error structure was tested to determine whether this structure improves the fit of the model for each outcome variable. This error structure simulates a decreasing correlation between data points as the time between them increases. To this end, two empty models were evaluated against one another: one wherein the correlation among residuals is the same across all variables, and one in which observations closer together in time are more highly correlated with one another (i.e., autoregressive error structure). A likelihood ratio test (LRT) was used to determine whether the latter model significantly improved the fit of the former model for 1) FR-VAS, and 2) FR-A. The LRT confirmed that the autoregressive error structure improved the fit of the model for both FR-VAS $\left[\chi^{2}(1)=18.5, p<.0001\right]$ and FR-A $\left[\chi^{2}(1)=20.5, p<.0001\right]$.

\subsubsection{Sleep Diary}

This section will outline the results of the statistical analyses used to test Hypotheses 1 (i.e., SQ-SD predicting FR-VAS) and 2a (i.e., TST-SD predicting FR-VAS). As two variables were used to predict FR-VAS, the correlation between SQ-SD and TST-SD was examined in order to assess for multicolinearity. SQ-SD and TST-SD were significantly correlated with one another $(\mathrm{r}=0.50, \mathrm{p}<.001)$. However, this correlation is moderate in strength and is not sufficiently large to suggest that multicolinearity is a problem in the current investigation (Pedhazur, 1997).

The intraclass correlation coefficient (ICC) serves as an index of the between- and within-person variability that can be explained by the model. More specifically, it assesses the 
degree of dependence, or the extent to which the data is nested, which can help to determine whether HLM is an appropriate technique for the data structure. The ICC for FR-VAS was 0.58 . This indicates that $58 \%$ of the variability is between-person, whereas $42 \%$ represents withinperson variability. Traditional analyses which primarily rely on between-persons variability would often not account for the within-person variance. Given that a substantial portion (i.e., $42 \%$ ) of the variance is within-persons, an HLM framework, which separates between- from within-person variability, is appropriate for this data.

In addition to the predictor variables of SQ-SD and TST-SD, several other variables which may have an effect on the outcome were also tested as potential predictors of fatigue. Specifically, as HLM analyses track participant's fatigue levels over time, the effects of time (i.e., day) were controlled for, in order to determine whether changes in participant fatigue levels (e.g., consistent increases or decreases in fatigue) across the two weeks would affect the outcome. The effect of time is a level 1 variable because it is looking at change in fatigue levels across time within individual participants. Nevertheless, when added into the model, day was not a significant predictor of FR-VAS, $B=-0.01, t(808)=-0.45, p=0.66$. Participant age, depression level, and group status (i.e., NS vs. ID), are potentially important level 2 predictors, and thus they too were entered into individual models to determine whether they contributed significantly to the variance. Depression was assessed via the depression subscale of the Depression Anxiety Stress Scales (DASS; $M=4.98 S D=6.87$ ). Age was not a significant predictor in the individual models, $B=-0.03, t(63)=-1.70, p=0.09$. Depression level $[B=-$ $0.13, t(63)=-3.93, p=.0002]$ and sleeper status $[B=-2.66, t(63)=-7.13, p<.0001]$ were significant, and were thus included in the final model, along with SQ-SD and TST-SD. 
Evaluating the study hypotheses. In order to determine whether SQ-SD and TST-SD predicts FR-VAS, two sets of variables were created. The first set simply involved the person means of SQ-SD, TST-SD and FR-VAS; that is, each person's mean score of SQ or TST across the 14 days. These variables represent level 2 as each participant has one mean across the two weeks. The second set of variables are person-mean centred variables, which model the daily fluctuations around the mean score for a given individual participant. These variables were created by subtracting the person's mean score on that variable from all of their daily ratings for that given variable; thus person-mean centred variables, by definition, have a mean of zero. Person-mean centred variables are characterized by level 1, as they are looking at the daily fluctuation of variables within participants.

Taken together, the person means of each variable represent each participants' mean SQ or TST across the two weeks, while the person-mean centred variables represent each individual's daily fluctuation around his or her own mean scores on these variables. The distinction between these two sets of variables allows us to easily distinguish between-person (i.e., level 2) from within-person (i.e., level 1) effects, respectively. Once again, level 2 analyses ask questions such as, "do people who generally sleep less report increased daytime fatigue?” Level 1 analyses, on the other hand, ask questions such as, "on nights in which a person sleeps less than their average total sleep time, do they also report higher levels of fatigue the following day?"

Table 6 presents the final model of SQ-SD and TST-SD predicting FR-VAS on the sleep diary. Depression level and sleeper status were included in the final model to determine whether they contributed to the variance; however, neither variable had a significant effect on the outcome. The level 1 results demonstrate that both SQ and TST on the sleep diary have 
Table 6

Final Mode of Sleep Diary Total Sleep Time and Sleep Quality predicting VAS Fatigue Ratings

\begin{tabular}{|c|c|c|c|c|c|}
\hline Fixed Effects & $B$ & $S E$ & $d f$ & $t$ & $p$ \\
\hline \multicolumn{6}{|l|}{ Within-person } \\
\hline TST-SD ${ }_{\text {centred }}$ & 0.15 & 0.05 & 795 & 2.90 & 0.004 \\
\hline SQ-SD centred & 0.64 & 0.10 & 795 & 6.10 & $<.0001$ \\
\hline \multicolumn{6}{|l|}{ Between-persons } \\
\hline Sleeper Status & -0.15 & 0.54 & 60 & -0.28 & 0.78 \\
\hline DASS-D & -0.05 & 0.03 & 60 & -1.87 & 0.07 \\
\hline TST-SD & 0.19 & 0.20 & 60 & 0.99 & 0.32 \\
\hline SQ-SD & 1.42 & 0.29 & 60 & 4.88 & $<.0001$ \\
\hline Random Effects & $B$ & $S E$ & & $Z$ & $p$ \\
\hline Error Structure & 0.19 & 0.04 & & 4.57 & $<.0001$ \\
\hline TST-SD ${ }_{\text {centred }}$ & 0.04 & 0.03 & & 1.45 & 0.07 \\
\hline SQ-SD centred & 0.26 & 0.11 & & 2.48 & 0.007 \\
\hline
\end{tabular}

Note. $\mathrm{VAS}=$ Visual Analog Scale, TST-SD = Total sleep time on the sleep diary, SQ-SD = sleep quality on the sleep diary, DASS-D = Depression subscale of the Depression Anxiety Stress Scales. 
significant within-person associations with VAS fatigue ratings. With respect to level 2 results, SQ, but not TST, has a significant between-person association with fatigue.

Contrast analyses. Given that at level 1, both SQ-SD and TST-SD were significant within-person predictors of FR-VAS, contrast analyses were performed to determine the relative size of the contributions. As SQ-SD and TST-SD are measured on different scales, with SQ measured on a five-point scale and TST measured as total hours and minutes of sleep, the slope coefficients were standardized in order to perform the contrast. Specifically, this test provides an estimated difference in cell means of each variable and tests whether or not this difference is equal to zero. The contrast revealed that SQ-SD produced a significantly larger level 1 effect compared with TST-SD, in its prediction of FR-VAS, $B=-0.10, t(808)=-2.43, p=0.015$.

\subsubsection{Actigraphy.}

This section will outline the results of the statistical analyses used to test Hypothesis $2 \mathrm{~b}$ (i.e., TST-A predicting FR-A). Forty-three participants' data (i.e., $66 \%$ of the total sample) was used to conduct the actigraphy analyses. This smaller sample size was a result of several participants' malfunctioning actiwatches as well as the elimination of participants' data that was unable to be scored. No significant differences were found between the total sample and participants who had usable actiwatch data on self-reported fatigue $[t(63)=1.88, p=.07]$ or selfreported insomnia $[t(62)=1.49, p=.14]$

The ICC for the actigraphy data was 0.75 , indicating that $75 \%$ of the variability is between-person and $42 \%$ represents within-person variability. These values suggest that an HLM framework is appropriate for this data. Day, age, depression level, and sleeper status variables were once again evaluated in individual models to determine whether they contributed significantly to fatigue ratings on the actiwatch. As was the case with the sleep diary, day $[B=-$ 
$0.0005, t(479)=-0.03, p=0.98]$ and age $[B=0.03, t(41)=1.49, p=0.14]$ did not contribute to the variance, whereas depression level $[B=0.16, t(41)=4.93, p<.0001]$ and sleeper status $[B=$ $0.14, t(41)=5.02, p<.0001]$ were significant predictor of fatigue ratings,

Evaluating the study hypotheses. As was done in the Sleep Diary analyses, person mean and person-mean centred variable were created for TST-A. Table 7 presents the final model of TST-A predicting FR-A, along with depression level and sleeper status. Depression level and sleeper status were both significant predictors of FR-A. Given that sleeper status was a significant predictor, the interaction between sleeper status and TST-A (i.e., which would examine whether the relationship between TST-A and FR-A differed between NS and ID) was included in the model. However, the interaction between sleeper status and TST-A was not significant. Neither the level 1 nor level 2 analyses for TST-A was significant in predicting FRA.

\subsubsection{Group Differences}

In order to test for group differences on levels of fatigue (i.e., Hypothesis 3), ID and NS were compared on their self-reported fatigue on the FSS. Those with ID were found to report significantly higher levels of fatigue compared to NS, $F(1,62)=69.07, p<.001, \eta^{2}=0.53$. In order to corroborate these findings, there was a significant main effect of sleeper status on FRVAS, $B=-2.66, t(63)=-7.13, p<.0001$. However, this effect was no longer significant in the final model, $B=-0.15, t(60)=-0.28, p=0.78$. There was a significant main effect of sleeper status on FR-A both in an empty model $(B=0.14, t(41)=5.02, p<.0001)$, as well as in the final model $(B=1.785, t(39)=3.21 p=.003)$. Finally, there was no significant interaction between sleeper status and FR-A in the final HLM model, $B=0.002, t(469)=1.20, p=0.23$. 
Table 7

Final Model of Actigraphy Total Sleep Time predicting Fatigue Ratings on the Actigraph

\begin{tabular}{lccccc}
\hline Fixed Effects & $B$ & $S E$ & $d f$ & $t$ & \\
\hline Within-person & & & & \\
TST-A centred $_{\text {Between-persons }}$ & -0.002 & 0.001 & 469 & -1.44 & 0.151 \\
Sleeper Status & 1.785 & 0.556 & 39 & 3.21 & 0.003 \\
DASS-D & 0.10 & 0.035 & 39 & 2.94 & 0.005 \\
TST-A & -0.00 & 0.003 & 39 & -0.19 & 0.847 \\
TST & & & & \\
Sleeptred & 0.002 & 0.002 & 469 & 1.20 & 0.230 \\
\hline Random Effects & $B$ & $S E$ & & $Z$ & $p$ \\
\hline Error Structure & 0.23 & 0.057 & & 4.08 & $<.0001$ \\
TST-A & & & & 0.80 & 0.212 \\
\hline
\end{tabular}

Note. TST-A = Total sleep time on the actigraph, DASS-D = Depression subscale of the Depression Anxiety and Stress Scale. 


\subsection{Mediation Analyses}

Bootstrapping mediation analyses were used to evaluate Hypothesis 4, which states that each of the cognitive-behavioural variables (i.e., DBAS, DISRS, FOF and AOF) would mediate the relationship between sleep, as measured by the ISI, and fatigue, as measured by the FSS. Bootstrapping (Preacher \& Hayes, 2004; 2008) was selected as the statistical procedure of choice as it overcomes many of the limitations inherent in the commonly used Causal Steps Approach first proposed by Baron and Kenny (1986). For example, bootstrapping has more statistical power associated with it and is better able to quantify the presence of indirect effects. In the current study, cognitive-behavioural mediators of the relationship between the ISI and FSS were tested; specifically: (1) DBAS, (2) DISRS, (3) FOF, and (4) AOF, were tested as mediators in four separate mediation models.

\subsubsection{Simple Mediation Models}

Each of DISRS, DBAS, FOF and AOF were tested as individual mediators in separate mediation analyses. The Depression subscale of the DASS was entered as a covariate in the former mediation model whereas the Stress and Anxiety subscales were entered as covariates into the latter three models. None of the covariates were significantly related to the dependent variables (i.e., the FSS), in each of the four models; thus they were excluded from the mediation analyses (Hayes, 2009). The relationship between FSS and ISI was significantly mediated by the DISRS $(\beta=.05, S E=.01,95 \%$ CI $[.030, .071])$, DBAS $(\beta=.07, S E=.02,95 \%$ CI $[.034, .109])$ and FOF $(\beta=.02, S E=.01,95 \% \mathrm{CI}[.003, .041])$. There was no significant indirect effect of ISI on FSS via AOF (95\% CI [-.002, .029]. 


\section{CHAPTER 5}

\section{DISCUSSION}

\subsection{Summary of Findings}

The study results provided support for Hypothesis 1, in that ratings of sleep quality on the sleep diary was a significant predictor of fatigue ratings. Specifically, lower ratings of sleep quality were related to higher ratings of daytime fatigue, and vice versa. This was true both within- and between-persons, and for both those with and without insomnia, as the analysis did not demonstrate significant group differences once total sleep time and sleep quality were accounted for in the model. At the within-person level (i.e., level 1), when a participant rated their quality of sleep higher than normal on a given night, they also tended to rate their fatigue as lower (i.e., higher energy) the following day. The between-person (i.e., level 2) results similarly suggested that participants who generally rated their sleep quality higher also tended to report lower levels of fatigue on the VAS. These findings are consistent with previous literature which supports the strong association between perceived sleep quality and reports of fatigue (FortierBrochu et al., 2010; Ustinov et al., 2010).

Partial support was found for Hypothesis 2a, which explored the relationship between total sleep time and daytime reports of fatigue on the sleep diary. Hypotheses $2 b$ was not found to be significant, as objective sleep time on the actigraph did not significantly predict prospective fatigue ratings the following day. This was true at both the within- and between-person level. That is, one night of objectively less total sleep time relative to average sleep length for a given individual did not contribute to increased fatigue ratings the following day. Similarly, those with overall lower objective total sleep times did not generally have increased ratings of fatigue on the actiwatch across the two weeks. Hypothesis 2 a (i.e., on the sleep diary) was significant at the 
within-person, but not at the between-person level. Thus, participants who tend to have shorter sleeps did not generally report more fatigue than those who obtained more sleep across the two weeks. By contrast, when a given individual reported obtaining less sleep than their average sleep length on a given night, the following day's fatigue rating on the VAS increased, and vice versa. For example, if an individual is accustomed to getting an average of seven hours of sleep per night, they would have higher fatigue ratings following a six-hour night of sleep, and lower fatigue ratings following an eight hour night.

As a result of the significant within-person findings that subjective ratings on both lower total sleep time and sleep quality on one night predicted increased fatigue the following day, a post-hoc contrast analysis was performed in order to compare the relative contribution of withinperson sleep length and sleep quality as predictors of VAS fatigue ratings. The results revealed that while both level 1 analyses were significant, sleep quality predicted VAS fatigue to a larger extent than did total sleep time.

The findings for Hypotheses 1 and 2 suggest that subjective ratings of sleep quality were superior to both objective and subjective total sleep time in the prediction of daytime fatigue. The between-person results found that while ratings of sleep quality predicted VAS fatigue ratings, participants who generally reported shorter sleeps did not have higher levels of daytime fatigue on both the sleep diary and actiwatch. Although the latter finding may seem counterintuitive, it is largely consistent with the research in this area. These findings are commensurate with the notion that there is not a consistently high correlation between total sleep time and daytime fatigue. Previous studies support these findings and have demonstrated that those with lower total sleep time or more disturbed sleep do not consistently report increased levels of fatigue (Alapin et al., 2000; Bonnet \& Arand, 1996; 1998; Fichten et al., 1995). These 
non-significant findings for total sleep time could be contrasted with the significant betweenperson findings for sleep quality and VAS fatigue ratings, wherein overall higher ratings of quality were associated with lower daytime fatigue. Indeed, taken together, these results suggest that while less total sleep does not necessarily lead to increased fatigue, poorer sleep quality ratings, and thus the perception of one's sleep, significantly predicts increased daytime fatigue.

The within-person findings, which provide estimates of the intra-individual variability, are somewhat more complex. Within-person analyses ask whether participants who get less sleep than is typical for them on one night also have increased fatigue the following day. These findings were significant for total sleep time and sleep quality on the sleep diary, but not for total sleep time on the actiwatch. The significant findings for sleep quality and non-significant finding for objective total sleep time are consistent with the notion that the perception of poor sleep has a greater influence on fatigue as compared to objective sleep time. Interestingly, within-person sleep length on the sleep diary was a significant predictor of VAS fatigue ratings, suggesting that on one night, participants who obtained less total sleep based on their sleep diary estimates were more likely to report increased fatigue the following day. There are several additional explanations that could account for this finding. First, it is important to note that while the sleep diary is the gold standard for measurement of sleep indices, it remains a subjective measure, in the sense that participants fill out the diary each morning based on their best estimate of their previous night's sleep. Therefore, as with all subjective measurements, it is possible that participants' perceptions of their sleep influenced their total sleep time estimates, and would have therefore been more likely to predict fatigue ratings. For example, if an individual woke up feeling unrefreshed, or feeling as though they had a poor quality of sleep, they may be more likely to overestimate their sleep onset latency, or the time spent awake in bed during the night, 
thereby underestimating their total sleep time. The fact that this significant within-person finding on the sleep diary was not corroborated by the actigraph data is important to note, as is the modest correlation between sleep diary and actigraphy estimates of total sleep time (i.e., $r=.29$ ). Indeed, these comparisons provide further support for the distinction between objective and subjective measurements of sleep, and emphasize the importance of using them both in investigations of this nature.

It is also possible that keeping a sleep diary for two weeks made participants more aware of whether they obtained more/less sleep than is typical for them on a given night. By recording the length of time it took to fall asleep and how long they spent awake in bed, in addition to estimating their total sleep time for the previous night, participants could be primed to think about whether they obtained more/less sleep than they usually would. This priming effect could conceivably influence their fatigue ratings. In developing the study design, this potential limitation was offset by having participants complete the VAS before bed, and thus a substantial amount of time would have passed since completing the morning sleep diary. Nevertheless, it is possible that priming could have contributed to the significant within-person, but not betweenperson findings for total sleep time and fatigue on the sleep diary. Furthermore, this could also explain why objective sleep time did not predict fatigue ratings on the actigraph, as it is less likely that participants would have been primed by simply wearing the actiwatch since they were not asked to record any information about their sleep for this index.

Both the within- and between-person findings on the sleep diary for total sleep time are consistent with the notion that nightly sleep needs is not a "one size fits all" approach, and that optimal amounts of sleep differ from person to person. Accordingly, individuals who obtain six hours of total sleep time, as opposed to eight hours, may not necessarily report more fatigue if 
they are accustomed to six hours; a finding which is consistent with the non-significant betweenperson results. However, if an individual obtains six hours of sleep but is accustomed to getting closer to eight hours of sleep, they are likely to report increased fatigue the following day. Indeed, this latter finding is in accordance with the significant within-person relationship between total sleep time and VAS fatigue ratings on the sleep diary. Implications for treatment that follow from this finding will be discussed in the subsequent section.

The results demonstrated that there was no association, at either the within- or betweenperson level, between objective total sleep time and prospective fatigue ratings on the actigraph. While this is the first study investigating the relationship between objective sleep and momentary fatigue ratings over time, previous research looking at the relationship between sleep and daytime indices across time, such as pain (O’Brien et al., 2011) and affect (McCrae et al., 2008) also found significant results for subjective sleep only, but not objective sleep. These findings highlight the distinction between subjective and objective measurements of insomnia, a discrepancy which has been well documented in the insomnia literature (Buysse et al., 1991; 2006; Vitiello et al., 2002). Like pain and affect, fatigue is a subjective experience, and it therefore makes sense that it would be more highly associated with subjective reports of sleep. The finding that objective assessments of sleep do not predict daytime fatigue further supports this idea that fatigue is more related to the perception of poor sleep as opposed to less total time spent sleeping during the night.

Hypothesis 3 was concerned with group differences (i.e., those with insomnia disorder vs. normal sleepers) with respect to levels of fatigue, as well as with respect to the relationship between sleep and fatigue. After controlling for age, insomnia sufferers had significantly higher self-reported fatigue (i.e., on the FSS) compared to normal sleepers. To further corroborate this 
finding, when sleeper status was entered as a covariate in the empty models, significant main effects of sleeper status were found for both the sleep diary and actigraph, suggesting that those with insomnia had significantly higher levels of fatigue on both VAS and momentary prospective ratings of fatigue on the actigraph. These results are consistent with the welldocumented findings that individuals with insomnia report experiencing more fatigue than normal sleepers (Fichten et al., 1995; Hossain et al., 2005; Lichstein et al., 1997; Moul et al., 2002). It also extends these findings, as the present study evaluated three different types of fatigue measurements; thus we can conclude that fatigue is higher among retrospective selfreport measures, daily retrospective VAS measures, as well as momentary prospective ratings which were collected three times per day on the actiwatch.

Despite the well-documented finding that individuals with insomnia report higher levels of fatigue than normal sleepers, the results of Objective 2 demonstrate that across both ID and NS groups, obtaining fewer hours of sleep did not necessarily predict higher ratings of fatigue. That is, individuals who may average six hours of sleep per night do not routinely report more daytime fatigue compared to those who average eight hours of total sleep time. Indeed, this finding was further supported as the interactions between sleeper status and both total sleep time and sleep quality were not significant in either model. Given that the number of hours of sleep obtained is likely not the primary reason for higher reports of fatigue, these findings suggest that it may be the perception of sleep quality, or perhaps the characteristics of an individual with insomnia, that contributes to higher levels of fatigue. For example, some people with insomnia tend to underestimate their sleep duration and are more likely to report poor sleep quality, even when sleep is objectively normal, or similar to those who do not complain of insomnia (McCrae et al., 2003; 2005; Means et al., 2000). This finding supports the overall contention of this study: 
that it is the preoccupation with and negative evaluation of sleep that characterizes those with insomnia, in addition to the objective sleep problem, which may or may not be present. As will be discussed in the next section, the maladaptive cognitive processes that are so common among those with insomnia can help us better understand the perceived association between poor sleep and fatigue.

The fourth hypothesis was concerned with what accounts for the increased fatigue in individuals with insomnia, as this increase cannot be solely due to disturbed or lack of sleep. The mediation analyses demonstrated that fatigue-based rumination, maladaptive beliefs about sleep, and fear of fatigue each significantly mediated the relationship between insomnia and selfreported fatigue. That is, each of these cognitive variables helps to account for the relationship between sleep and fatigue among those with and without insomnia. Thus, the ruminating about daytime symptoms of fatigue (e.g., "think about how hard it is to keep my mind on task"), holding maladaptive beliefs about sleep (e.g., "I need 8 hours of sleep to feel refreshed and function well during the day"), and having a fear of fatigue (e.g., "fear of feeling 'foggy headed"') are integral to this well-documented association between sleep and fatigue.

Avoidance of fatigue was not a significant mediator of the relationship between sleep and fatigue. Similar findings were reported in a previous investigation exploring the fear and avoidance of fatigue constructs and the development of their scales. In this study, the AOF was not correlated with insomnia, and generally did not emerge as an appropriate scale for the measurement of avoidance of fatigue (Harris et al., submitted). One possible explanation for this finding is that AOF explores avoidance of symptoms and cues associated with fatigue; it is therefore a measure exploring behaviour (i.e., tendencies to engage in/avoid symptoms of fatigue). The significant mediators explore rumination, beliefs, and fears around sleep and 
fatigue, and are thus cognitive constructs. Given the argument throughout this paper that the experience of fatigue is largely based on perception of sleep, it makes sense that the cognitivebased measurements would be more likely to mediate the relationship between sleep and fatigue, as compared to measures assessing behavioural avoidance. Furthermore, it is possible that people with insomnia simply believe that fatigue is inevitable and do not believe they can avoid it. Thus, perhaps other measures, such as Ree and Harvey's (2004) Sleep-Related Safety Behaviours Questionnaire, which asks about specific behaviours people engage in to avoid the experience of fatigue (i.e., cancel daytime activities), are better able to capture avoidance of fatigue in clinical insomnia.

\subsection{Theoretical and Clinical Implications}

The results of this study have important implications for theoretical models of insomnia. Harvey's (2002) cognitive model of insomnia emphasizes the role of cognitive processes in the maintenance of sleep problems. The findings of the current investigation support this model in several ways. First, our results suggest that perceived quality of sleep is a better predictor of daytime fatigue than is reduced total sleep time during the night. Indeed, perception is one of the cognitive processes to which Harvey alludes in the maintenance of insomnia and associated daytime impairments. She posits that negatively toned cognitive activity, increased arousal and distress, and subsequent selective attention to sleep threats all contribute to a distorted perception of both the sleep deficit and daytime fatigue, which continues to perpetuate the problem.

The cognitive mediators (i.e., maladaptive sleep beliefs, fatigue-based rumination, and fear of fatigue) also fit nicely in this model. Maladaptive beliefs about sleep are proposed to exacerbate the negatively toned activity, as holding unrealistic expectation about one's sleep or daytime functioning can lead to worry and psychological distress when these expectations are 
not fulfilled. Fear of fatigue is thought to play an important role in the pre-sleep period, during which time poor sleepers are anxious about the potential daytime consequences of sleep loss. Therefore, these individuals can be said to "fear the fatigue" they may experience as a result of a poor night of sleep. Finally, the current study demonstrated that fatigue-based rumination plays the most important role in accounting for the relationship between sleep and fatigue. The model demonstrates that selectively attending to daytime symptoms of insomnia (e.g., fatigue, lack of concentration, low mood) leads patients to overestimate the extent of their daytime impairment, and increases the likelihood that they will attribute these symptoms to their sleep problem due to their preoccupation with sleep. As discussed in the introduction, this attribution tendency heightens the perception that the fatigue is caused by the preceding night's sleep, thereby enhancing the perceived association between sleep and fatigue.

Lundh and Broman (2000) also recognize that perception, or misperception, of sleep is fundamental to the maintenance of insomnia. Their model posits that misperception of total sleep time, unhelpful beliefs about sleep, and erroneous attributions about sleep and daytime functioning are all maladaptive sleep-interpreting processes, which in turn magnify the sleep problem. This particular model places additional emphasis on the attributions of nighttime and daytime symptoms, which coincides well the current study's findings that fatigue-based rumination, and the tendency to attribute daytime symptoms to the previous night's sleep, is key in the maintenance of insomnia and the relationship between sleep and fatigue.

The results of this study have important clinical implications for the administration of cognitive behaviour therapy for insomnia (CBT-I). CBT-I is the first-line treatment for insomnia, given its well-documented empirical support in both primary and secondary insomnia populations (Edinger, Wohlgemuth, Radtke, Marsh \& Quillian, 2001; Jacobs, Pace-Schott, 
Stickgold, \& Otto, 2004; Lichstein et al., 2001; Lichstein, Wilson, \& Johnson, 2000; Morin et al., 2006). While CBT-I has been deemed the gold standard treatment for insomnia, there remains a subset of patients who do not remit following treatment (Harvey \& Tang, 2003; Morin et al., 1994). Even more troubling is that once sleep improves with CBT-I, daytime functioning indices, including fatigue and quality of life, often do not change post-treatment (Means et al., 2003; Morin et al., 2006). Given that daytime impairments, such as fatigue, are amongst the most distressing symptoms of insomnia, efforts to improve CBT-I's impact on daytime functioning are warranted. Indeed, previous research has discussed the need to examine the effectiveness of CBT-I with respect to areas of life aside from sleep, such as daytime impairments and fatigue (Edinger \& Means, 2005).

The current study's finding that, compared to sleep length, perception of sleep is a better predictor of daytime fatigue is helpful for CBT-I therapists and patients alike. In CBT-I, the objective is not foremost to increase total sleep time on the sleep diary, but instead to increase sleep efficiency (i.e., the percentage of time in bed that one is sleeping) and perceived quality of sleep. Therefore, improvements in CBT-I can happen without increasing total sleep time, but instead shortening the time spent in bed in order to match the sleep needs of the patient. To be sure, treatment has been shown to improve perception and quality of sleep, while at the same time produces modest or even no apparent changes in objective sleep time (Morin et al., 1999; Murtagh \& Greenwood, 1995). As these findings are in agreement with the current study's results, the onus is on the therapist to educate patients about the nature of the relationship between sleep and fatigue, and teach them that there is not a one-to-one correspondence between the two. This study also demonstrates that holding erroneous sleep beliefs, ruminating about fatigue, and being fearful of fatigue all help account for the perceived relationship between sleep 
and fatigue. Modifying such cognitive processes may be important for reducing the perception of fatigue. Indeed, as will be discussed below, the results of this study can provide us with some helpful suggestions for addressing such maladaptive beliefs in CBT-I.

One belief about sleep, held among both insomnia sufferers and normal sleepers, is that a certain number of hours of sleep (e.g., eight) are needed each night in order to function the following day (Carney \& Edinger, 2006; Morin et al., 1993). The results of this study provide empirical evidence against this belief. The between-person results of the relationship between total sleep time on the sleep diary and VAS fatigue ratings showed that daytime fatigue did not vary based on number of hours of sleep. Rather, the results demonstrate that as opposed to this "one size fits all" approach, individuals have their own sleep needs that indeed may vary quite a bit between persons. As CBT-I is a very individualized treatment, wherein therapists work individually with patients on their sleep diaries, it is the job of the therapist and patient to collaboratively determine a suitable time in bed for the patient. The finding that people's sleep needs vary considerably and that average total sleep time does not necessarily predict daytime fatigue can potentially alleviate some of the sleep-related anxiety, and more specifically relieve some of the pressure to sleep commonly found among people with sleep difficulties.

Another problematic belief about sleep is the pervasive notion that daytime fatigue is caused by the previous night's sleep. The present study shows that the relationship between sleep and fatigue is indeed more complex than this one-to-one correspondence and is strongly influenced by cognitive processes and perception. Indeed, fatigue-based rumination, which often takes the form of attributing negative daytime symptoms (i.e., fatigue) to the previous night's sleep, was found to play the a very important role in the relationship between sleep and fatigue. 
This process of attributing fatigue solely to a poor night's sleep will again lead to negative cognitive activity related to sleep and perpetuate the insomnia cycle.

While interventions addressing maladaptive beliefs about sleep are incorporated into some CBT-I treatment manuals, maladaptive attributions related to sleep and fatigue are not currently targeted in treatments for insomnia. In light of the findings suggesting that misattributing fatigue to poor sleep can increase sleep-related anxiety and further perpetuate the insomnia (Harvey, 2002; Lundh \& Broman, 2000), addressing such attributions may prove to be a helpful adjunct to CBT-I. One possible intervention to counteract this attribution tendency would be to educate patients about the other common causes of fatigue, aside from poor sleep. Indeed, while insomnia sufferers tend to assume that lack of sleep is the most likely cause of daytime fatigue, research has demonstrated that there are a multitude of causes of fatigue, many of which are unrelated to sleep. Some particularly common examples include boredom (Grandjean, 1979), physical under- or over-activity (Puetz, O'Connor, \& Dishman, 2006), illnesses such as a virus or anemia (Sobrero et al., 2001), depression and anxiety (Greenberg, 2002), and caffeine withdrawal (Juliano \& Griffiths, 2004). Educating patients about these alternative factors could decrease their preoccupation with sleep, thereby reducing the likelihood that one would ruminate on the previous night's sleep. A previous investigation found that people's attributions for fatigue are amenable to change once they were provided with psychoeducation about these other possibilities for the causes of fatigue (Harris \& Carney, 2012). The current investigation provides further support for the use of such interventions in treatment for insomnia in order to lessen preoccupation with sleep as well as minimize the perceived association between poor sleep and daytime fatigue. 


\subsection{Study Strengths and Limitations}

There are several strengths and limitations associated with this study. One major strength is the inclusion of both clinical insomnia and normal sleeper groups. Both study samples were well-screened, as validated clinical interviews, including the SCID-I and DSISD, were used to determine participant eligibility into either group. Furthermore, each group contained equal numbers of participants and neither group met criteria for specific comorbid psychiatric disorders (e.g., Bipolar Disorder, Schizophrenia) or occult sleep disorders (e.g., Sleep Apnea, Circadian Rhythm Disorder) which could potentially interfere with the insomnia diagnosis. Whereas those who met criteria for depression and anxiety were included, these comorbidities were controlled for in the relevant analyses. Incorporating both a clinical insomnia and normal sleeper group presents an important advantage of this investigation. Although we did not predict that there would be significant group differences for several of the primary hypotheses, it was important to include both groups in order to test for group differences. For example, it was interesting to see that although insomnia sufferers had shorter total sleep times and higher ratings of fatigue, individuals with shorter sleep lengths (i.e., either those with ID or NS) did not routinely predict increased daytime fatigue.

The demographic characteristics represented in the study sample present some limitations worthy of mention. One potential problem is that the insomnia group was significantly older than the normal sleepers. Insomnia symptoms certainly vary across the lifespan, in that older individuals are found to report experiencing more sleep disturbances than their younger counterparts (Ancoli-Israel, 1997; Webb, 1989). Another possibility for the age discrepancy is that many good sleepers were students, as flyers were posted on Ryerson University's campus. We offset this potential limitation by controlling for age in each of the analyses which separated groups. Age was also included as a covariate in the multilevel models, though it was not 
significantly related to the outcome variables. Both the insomnia and normal sleeper groups were predominantly female (ID $=75 \%, \mathrm{NS}=88 \%$ ), which could present another challenge for the generalization of our results. However, the female predominance is not uncommon in either sample, as insomnia is more prevalent among women (Zhang \& Wing, 2006) and previous experience has shown that female students are more likely to participate in research studies at Ryerson University. Despite this potential drawback, the fact that the gender distributions in both groups were not significantly different can be considered an advantage, as group comparisons were unlikely to reflect gender differences. Future research in this area could aim to recruit an equal number of male and female participants from across the lifespan. Depending on the research question, it may be beneficial to match participants according to age and sex in order to control for these demographic characteristics, and the impact that they may have on study findings.

With regard to the study sample, another potential limitation was that those with ID were treatment seekers, whereas the NS group members were being paid for their involvement in the study. Thus, it is likely that the motivation for participating in the study differed depending on group status. Nevertheless, participants in both groups were being offered something of value in return for their participation in the study. Providing treatment to the ID group and financial compensation to the NS group was deemed to be the most fair and ethical way in which to compensate all participants wishing to be involved in the current study.

It is important to acknowledge the limitations surrounding the use of mediation in crosssectional data. Although this study employed time-series analyses to investigate the primary research question, the secondary analyses, for which we used mediation, was cross-sectional in nature, as the data were only collected at one time point. Indeed, cross-sectional examinations of 
mediation can potentially bias the results and can either over- or under-estimate longitudinal or causal processes which cannot be directly tested by these analyses (Maxwell \& Cole, 2007). Given these potential biases, it is important to note that as is the case with many tests of statistical mediation, the mediation analyses in the present study cannot speak to causal processes among the variables. Future research can consider collecting mediational data across several time points in order to obtain a better understanding as to how the relationship between these processes evolve over time.

Although we controlled for depression and anxiety in the HLM and mediation analyses, it is important to note that there are several other variables which could influence the experience of fatigue, in addition to sleep variables, which were not controlled for in the present study. For example, caffeine intake, amount of physical activity, and nutritional intake may all influence individuals' varying levels of fatigue throughout the day. However, these aforementioned factors represent state variables, whereas anxiety and fatigue are trait measures. In the current study, we were primarily interested in the latter, given the relevance and potential interaction of trait-based psychological factors, such as depression and anxiety, with sleep and fatigue processes.

Furthermore, given the infinite number of such state variables, and the unpredictable nature by which these variables may influence fatigue at any given time throughout the day, it was not feasible to assess these potential confounds in the present study. Nevertheless, given that the findings suggest that sleep quantity itself may not be as central to the experience of fatigue as once thought, these additional state-based variables and the degree to which they may impact on fatigue levels may warrant investigation in future studies examining fatigue and its correlates.

The use of actigraphy data in the current study posed several limitations that are worthy of discussion. First, 23 participants were excluded from the actigraphy analyses, either as a result 
of malfunctioning actiwatches or refusal to wear the watch. In the majority of cases, the watch malfunctioned as the alarm reminder to input the fatigue ratings on the watch did not sound. In these cases, individuals either did not enter any fatigue ratings or in rare circumstances, they remembered to enter their fatigue ratings on the first day without the alarm prompt. However, this data was not used in the final analyses, as participants did not enter their fatigue ratings at the designated times (i.e., $10 \mathrm{am}, 1 \mathrm{pm}$, and $7 \mathrm{pm}$ ), and therefore the data were not considered to be reliable. For some participants, the actiwatch was deemed uncomfortable and was therefore not worn for the majority of the two week period. Participants that were excluded from analyses in these cases did not have a consecutive day and night of usable data. Nevertheless, participants who did and did not wear the actiwatch were not found to differ significantly on self-reported fatigue or insomnia. Furthermore, the multilevel modeling analyses, which were used to analyze the actigraphy data, are known to be quite robust against small sample sizes. This is primarily due to the fact that 14 days and nights of data were collected for each participant, resulting in considerably more data points to analyze than would be the case in a cross-sectional study with the same number of participants.

Second, and perhaps more importantly, the actigraphy data suggests that the actiwatches underestimated total sleep time in both good sleepers and those with insomnia. This underestimation is probable due to the comparison with sleep diary total sleep time values in the current study (i.e., sleep diary mean TST $=6.64$ hours, actigraphy mean TST $=5.21$ hours) as well as previously reported values of total sleep time for good and poor sleepers in the literature. The insomnia literature has found that low correlations exist among various measurements of sleep, including self-report questionnaires, sleep diaries, actigraphy, and polysomnography (Buysse et al., 2006; McCall \& McCall, 2012). With specific reference to actigraphy, in their 
recommendations for standard research assessment of insomnia, Buysse and colleagues acknowledged that this tool was the most controversial of all the sleep measures they assessed. Nevertheless, actigraphy is the only feasible way to monitor objective sleep across two weeks in participants' home environment. Thus, despite its limitations, it was determined that this was the most appropriate tool currently available to provide the objective measurement of sleep required in this study.

In the current investigation, it is possible that the algorithm used for our actigraphs may have been too sensitive and mistakenly captured slight movements as time spent awake in bed. It is encouraging, however, that the values for total sleep time on the actiwatch correlated significantly with total sleep time on the sleep diary. Furthermore, total sleep time on the actigraph was able to distinguish poor from good sleepers, as the former group obtained significantly less total sleep compared to the latter. Thus, it appears that the underestimation of total sleep time on the actigraph was systematic and reliable across participants and both groups. Nevertheless, given this consistent underestimation of total sleep time, it may have been advisable to pilot test the actiwatches for their reliability of sleep estimations prior to using them in the study.

Although the use of the actigraph posed some challenges, perhaps the most important advantage of this study is its use of both subjective and objective indices of sleep, as well as retrospective and prospective measurements of fatigue. This multi-method approach was integral to the current study, as any comprehensive investigation into the relationship between sleep and fatigue would need to distinguish between subjective and objective reports of sleep. Previous research has certainly highlighted the importance of utilizing different types of measurements when studying sleep (McCrae et al., 2008). As there are notable strengths and weaknesses of 
many insomnia assessment tools, it has been recommended to use a variety of measurements as complementary in research (Buysse et al., 2006). Furthermore, subjective and objective sleep measurement tools have been found to poorly correlate with one another (Espie et al., 1989; McCrae et al., 2005; Means et al., 2003), and the modest correlation in the current study between total sleep time on the sleep diary and actigraphy is no exception to this finding. These findings further demonstrate the need to include both objective and subjective measures of sleep in the current investigation.

In addition to the multi-method assessment of sleep employed in the current study, fatigue was also examined in a variety of ways. As fatigue is a subjective construct, it is most commonly measured via self-report questionnaires and VAS. In conjunction with these assessment tools, the present study also measured fatigue prospectively using Likert scale fatigue ratings which were entered three times per day on the actiwatch. Instead of measuring fatigue once per day, fatigue levels were assessed three times daily and the mean of each day was used in order to control for time of day effects. As previously mentioned, this was done to ensure that all participants recorded fatigue at the same time points and to limit the impact of welldocumented elevations of fatigue throughout the day, such as right after awakening (i.e., due to sleep inertia) and after lunch (i.e., due to the post-lunch dip in body temperature), on participant ratings. To our knowledge, this is the first investigation to assess fatigue prospectively in this manner. Without being able to assess fatigue objectively, this method was thought to circumvent, to the extent that is possible, individual bias and perception which can often confound assessments of fatigue. 


\subsection{Summary and Conclusion}

Given that daytime fatigue is one of the most distressing and debilitating consequences of insomnia (Buysse et al., 2007; Moul et al., 2002), research that explores the daytime sequalae of sleep disturbance is critically important work. This study has importantly found that less total sleep time does not invariably lead to increased fatigue, and that subjective sleep quality is a more important predictor of fatigue than is sleep quantity. Furthermore, what we think about our sleep (e.g., our beliefs about sleep and how we perceive our fatigue symptoms) plays an integral role in the relationship between sleep and fatigue. As individuals with insomnia continue to place undue pressure on themselves to obtain a "good" night of sleep in order to function the next day, the results of this study could help decrease this burden, and thereby lessen the overall sleeprelated anxiety. Furthermore, as noted previously, while CBT-I is currently the gold-standard treatment for insomnia, fatigue and associated daytime impairments often do not remit with treatment (Means et al., 2003; Morin et al., 2006). These finding suggest that developing and testing fatigue-based adjuncts to CBT-I, particularly those which emphasize maladaptive cognitions, may be a worthwhile clinical endeavor.

It is hopeful that this study inspires further inquiry into the study of fatigue in those with insomnia. As this research is still in its infancy, there continues to be much to learn with regard to the impact of fatigue on the lives of patients with insomnia. The present study's findings suggest that it is the perception of poor sleep, as opposed to the quantity of sleep, which has the major impact on daytime fatigue. Moving forward, treatment protocols and future research studies alike should continue to explore maladaptive cognitions in sleep disturbed populations to gain further knowledge as to their role in the debilitating condition of insomnia. 


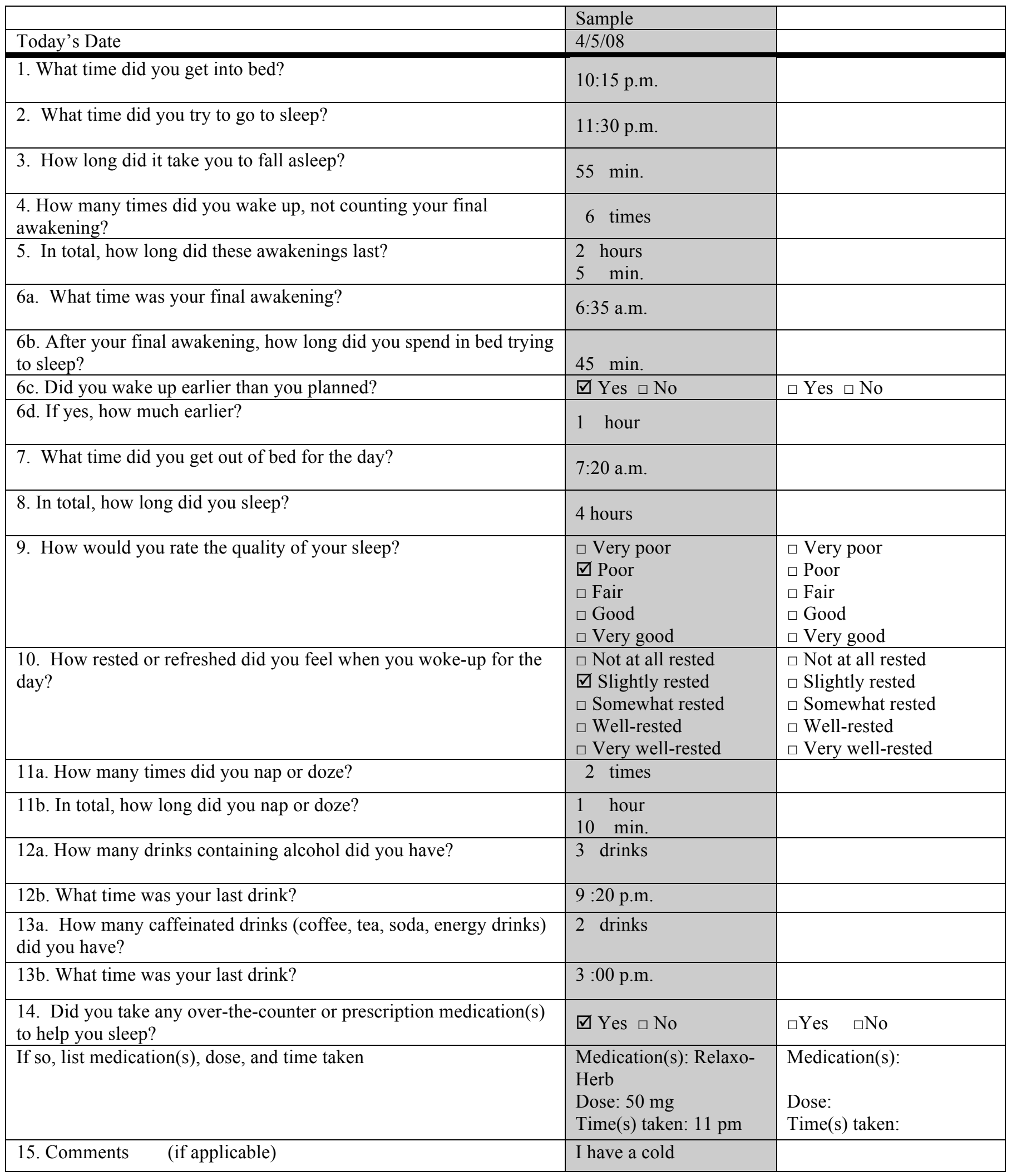




\section{Appendix B}

Visual Analogue Scale

How fatigued/energized did you feel today? Please mark an " $X$ " on the line below to indicate how you felt today.

Extremely fatigued — Extremely energized 


\section{Appendix C}

FOF/AOF

Please circle the number that best indicates the extent to which you fear and avoid the following experiences.

$$
\begin{aligned}
& 0=\text { Not at all afraid/avoid } \\
& 1=\text { A little } \\
& 2=\text { A fair amount } \\
& 3=\text { Much } \\
& 4=\text { Very much afraid/avoid }
\end{aligned}
$$

FEAR

\section{$\begin{array}{lllll}0 & 1 & 2 & 3 & 4\end{array}$}

$\begin{array}{lllll}0 & 1 & 2 & 3 & 4\end{array}$

$\begin{array}{lllll}0 & 1 & 2 & 3 & 4\end{array}$

$\begin{array}{lllll}0 & 1 & 2 & 3 & 4\end{array}$

$\begin{array}{lllll}0 & 1 & 2 & 3 & 4\end{array}$

$\begin{array}{lllll}0 & 1 & 2 & 3 & 4\end{array}$

$\begin{array}{lllll}0 & 1 & 2 & 3 & 4\end{array}$

$\begin{array}{lllll}0 & 1 & 2 & 3 & 4\end{array}$

$\begin{array}{lllll}0 & 1 & 2 & 3 & 4\end{array}$

$\begin{array}{lllll}0 & 1 & 2 & 3 & 4\end{array}$

$\begin{array}{lllll}0 & 1 & 2 & 3 & 4\end{array}$

$\begin{array}{lllll}0 & 1 & 2 & 3 & 4\end{array}$

\section{AVOID}

$\begin{array}{lllll}0 & 1 & 2 & 3 & 4\end{array}$

1. Exercising for long periods of time

$$
\begin{array}{lllllll}
0 & 1 & 2 & 3 & 4 & \text { 2. Staying up all night }
\end{array}
$$

$\begin{array}{lllll}0 & 1 & 2 & 3 & 4\end{array}$

3. Getting up early in the morning

$\begin{array}{lllllll}0 & 1 & 2 & 3 & 4 & 4 \text {. Feeling "foggy-headed" }\end{array}$

$\begin{array}{lllll}0 & 1 & 2 & 3 & 4\end{array}$

5. Dozing-off (i.e., falling asleep) in public

$\begin{array}{lllll}0 & 1 & 2 & 3 & 4\end{array}$

6. Looking so sleepy that people might notice

$\begin{array}{llllll}0 & 1 & 2 & 3 & 4 & \text { 7. Feeling mildly tired }\end{array}$

$\begin{array}{lllll}0 & 1 & 2 & 3 & 4\end{array}$

8. Feeling moderately tired

$\begin{array}{llllll}0 & 1 & 2 & 3 & 4 & 9 \text {. Feeling extremely tired }\end{array}$

$\begin{array}{lllll}0 & 1 & 2 & 3 & 4\end{array}$

10. Feeling like your limbs are heavy

$\begin{array}{lllll}0 & 1 & 2 & 3 & 4\end{array}$

11. Feeling like you can't concentrate

$\begin{array}{lllll}0 & 1 & 2 & 3 & 4\end{array}$

12. Feeling like you can barely keep your eyes open 


\section{Appendix D}

\section{DISRS}

People think and do many different things when they feel low. Please read each of the items below and indicate whether you almost never, sometimes, often, or almost always think or do each one when you feel down or tired. Please select only one answer. Please indicate what you generally do, not what you think you should do.

$$
\begin{aligned}
& 1=\text { Almost Never } \\
& 2=\text { Sometimes } \\
& 3=\text { Often } \\
& 4=\text { Almost Always }
\end{aligned}
$$

1234 1. Think, "I won't be able to do work because I feel so bad"

$\begin{array}{lllll}1 & 2 & 3 & 4 & 2 \text {. Think about your feelings of fatigue }\end{array}$

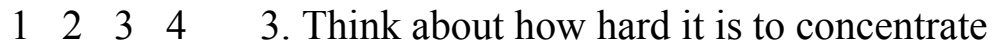

$\begin{array}{lllll}1 & 2 & 3 & 4 & 4 \text {. Think about how unmotivated you feel }\end{array}$

$\begin{array}{lllll}1 & 2 & 3 & 4 & 5 \text {. Think about how your thoughts are cloudy/muddled }\end{array}$

$\begin{array}{lllll}1 & 2 & 3 & 4 & 6 \text {. Think about how everything requires more effort than usual }\end{array}$

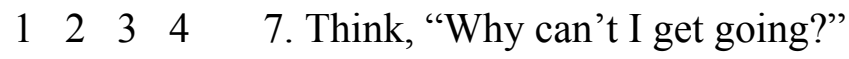

$\begin{array}{lllll}1 & 2 & 3 & 4 & 8 \text {. Think about how sad you feel }\end{array}$

$\begin{array}{lllll}1 & 2 & 3 & 4 & 9\end{array}$. Think about how you don't feel up to doing anything

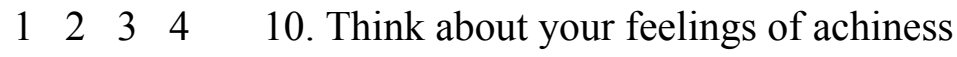

$\begin{array}{lllll}1 & 2 & 3 & 4 & 11 \text {. Think about how bad you feel }\end{array}$

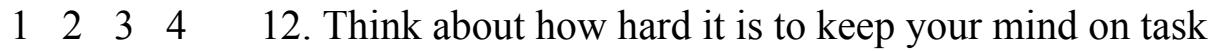

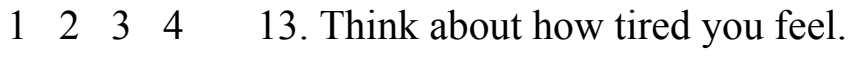

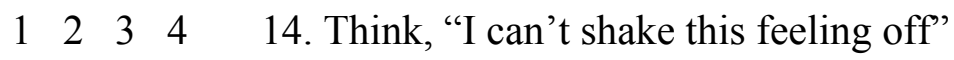

$\begin{array}{lllll}1 & 2 & 3 & 4 & 15 \text {. Think about how irritable you feel }\end{array}$

$\begin{array}{lllll}1 & 2 & 3 & 4 & 16 \text {. Think about how sleepy you feel }\end{array}$

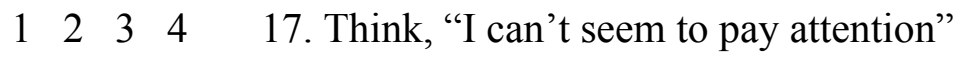

1234 18. Think, "I'm so forgetful"

1234 19. Think, "I can't be around people when I'm feeling this way"

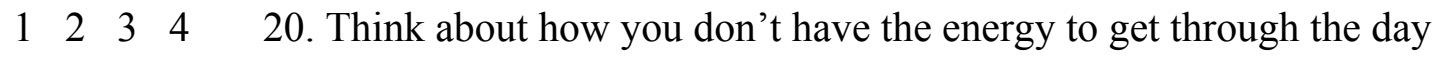




\section{Appendix E}

\section{Ryerson University Consent Agreement}

\section{Insomnia Treatment Study}

You are being asked to participate in a research study. Before you give your consent to be a volunteer, it is important that you read the following information and ask as many questions as necessary to be sure you understand what you will be asked to do.

\section{Investigators:}

Principal Investigator, Colleen E. Carney, Ph.D., Ryerson University

Co-Investigator, Taryn Moss Atlin, M.A., Ryerson University

Co-Investigator, Andrea Harris, M.A., Ryerson University

\section{Purpose of the Study:}

The purpose of this study is to compare two different treatment strategies for people with insomnia, and to determine how best to treat symptoms commonly associated with this disorder. Approximately 200 people meeting diagnostic criteria for insomnia (18-74 years of age) will be enrolled in this study. We will enroll those who are otherwise physically and emotionally healthy, and without any conditions that would interfere with their safe participation.

\section{Description of the Study:}

If you agree to be in this study, you will be asked to sign this consent form. You will be asked to participate in an interview (1 hour) that consists of a Mental Status Exam and structured sleep and psychiatric interviews at the Ryerson Sleep and Depression Laboratory. Your eligibility will be based on both your screening interview as well as the results of your sleep monitoring, which will be determined in your second visit. If you qualify for the study based on the results of the screening interview, you will be asked to take part in a number of optional study procedures and visits as outlined below:

\section{Screening Assessment Part 1}

- Upon completing the initial assessment, you will be asked to participate in a 15minute imaginal fatigue task, whereby you will be instructed to imagine the experience of fatigue and answer some questions while experiencing this transient imaginal state.

- You will also be asked to do some self-monitoring tasks over the next two weeks before you will qualify to begin the first treatment session, which will involve:

- Completing a sleep log each morning in your home (about 5 minutes) for two weeks to answer questions about the previous night's sleep.

- Completing an activity log each evening in your home (about 5 minutes) for two weeks to answer questions about your daytime activities.

- Wearing a small wristwatch-sized device called an actigraph for one week to measure your sleep at night and rate your fatigue three times per day when the alarm on the device sounds. You will be taught how to use this device by the 
study staff and will be asked to wear it at all times (even when you sleep), with the exception of the times when you are bathing or when you believe your wrist would be exposed to water.

- You will be asked to allow a study staff to take your waist circumference, as well as your height and weight in order to measure body mass index.

\section{Screening Assessment Part 2}

- Prior to beginning the first treatment session, you will be asked to complete a questionnaire package at the Ryerson Sleep and Depression Laboratory. These questionnaires will take approximately 30 minutes and will ask about your mood, sleep, and how you respond to sleep symptoms.

\section{Treatment Procedures}

- Following the baseline assessment, you will be randomly assigned (like drawing names out of a hat) into one of two study groups. Both groups will receive cognitive behavioural therapy for insomnia, but one group will also receive some elements of cognitive behavioural therapy for fatigue. More information about the treatment follows:

1. You will meet with a therapist at the Ryerson Sleep and Depression Laboratory biweekly for 4 sessions. Your therapist will provide information about strategies for managing your sleep. These visits will last approximately one hour each.

2. Completion of a sleep log each morning in your home (about 5 minutes) to answer questions about the previous night's sleep.

3. Completion of a therapy evaluation questionnaire on the first and last week of treatment (less than 5 minutes).

\section{Endpoint Assessment}

- The endpoint assessment will include some of the same procedures as the baseline assessment you completed before being assigned to a study group. It will include the following procedures:

- Completion of an outcome questionnaire packet (1 hour) at the Ryerson Sleep and Depression Laboratory.

- Participation in the 15-minute imaginal fatigue task

- Completing a sleep log each morning in your home (about 5 minutes) for four weeks to answer questions about the previous night's sleep.

- Completion of a one-week period using the actigraph device

\section{One-Month Follow-up}

- One month after the endpoint assessment, you will be asked to come back for a follow-up assessment. The procedures that will occur at the follow-up are:

- Completion of an outcome questionnaire packet (1 hour) at the Ryerson Sleep and Depression Laboratory.

You will be in the study for approximately 4 months. This includes a follow-up period of one month after the last therapy session. You can choose to stop participating at any time. 


\section{What is Experimental in this Study:}

None of the procedures or questionnaires used in this study are experimental in nature; that is, they are all accepted and regularly used treatments. The only experimental aspect of this study is the particular way that the elements of this treatment are being combined/presented.

\section{Risks or Discomforts:}

You may experience some anxiety and discomfort during the treatment because you are being asked to discuss your worries and concerns about sleep. For example, some of the questions we will ask you as part of this study may make you feel uncomfortable. In addition, you may experience embarrassment or discomfort when height, weight, and waist circumference are measured by study staff to assess for the proxy parameters of metabolic syndrome. In order to circumvent or minimize possible discomfort, you will be given several options for this procedure. For example, you do not have to bare your skin or you can choose to gather these measurements yourself after careful verbal direction. You may also choose not to engage in these procedures at any time without impunity. Although these risks are minimal, you are free to withdraw from participation at any time if this becomes too distressing.

You may refuse to answer any of the questions and you may take a break at any time during the study. You may stop your participation in this study at any time. You find it uncomfortable to wear the wristwatch device, particularly if you normally do not wear a wristwatch. Wearing the wristwatch device is voluntary. You may feel increased sleepiness during your treatment. This is most often brief (lasting a week or two at the most) and mild.

You will be regularly assessed for any worsening in your symptoms to determine if you can safely proceed through the study. If you or the Investigator determines your symptoms are worsening, you will meet to discuss whether it is in your best interest to be withdraw from the study. In cases wherein there are safety concerns, you could be removed from the study. If you and/or the Investigator decide that it is in your best interest to leave the study, you will be provided with referrals for alternative treatment.

\section{Benefits of the Study:}

You may experience a reduction in your sleep problems through participation in this study. Research has shown that cognitive behavioural therapy is an effective way to treat insomnia. However, there is no guarantee that your insomnia will improve from participating in this study. In addition, the anticipated benefit of this study is that the information learned from this study will inform us as to the best possible way to treat this condition, so that future patients with your condition will benefit from more effective therapies.

\section{Alternative Methods of Treatment:}

The treatment used in this study is part of conventional ways of treating insomnia and fatigue. Other alternatives to this treatment include drug therapy (in lieu of the psychotherapy used in this study). This alternative may be available or advantageous to you, and is generally available through a referral from your family doctor.

\section{Confidentiality:}


Every effort will be made to ensure confidentiality of any identifying information that will be obtained in connection with this study as required by law. Except when required by law, you will not be identified by name, address, telephone number, or any other direct personal identifier in study records disclosed outside of Ryerson University. Your data will be kept in a locked file in the Project Coordinator's office at Ryerson University. While the information and data resulting from this study may be presented at scientific meetings or published in a scientific journal, your identity will not be revealed.

Your study contains a unique code and this code will be used so that no identifying information will be tied to your study information. We will maintain some identifying information in a password-protected database on the Project Coordinator's computer in a locked office. This separate database will contain your name and contact information only. Only study related personnel have access to your personal health information, all of whom are bound to confidentiality by the ethics regulations of the profession of psychology. They will have access to your name for the purposes of addressing you personally during therapy, and communicating any safety concerns with the Principal Investigator.

Audio tapes will be used to record therapy sessions. These tapes will be kept in a locked cabinet at the Ryerson Sleep and Depression Laboratory. They will be labelled by code, rather than name. The purpose of these recordings is to rate the interviewer and the therapist to ensure that these procedures are carried out competently. The recordings will not be used in any publications. Only study personnel will have access to this information. Confidentiality will be maintained to the extent allowed by law for 10 years after the study's completion. The recording will be stored for 10 years after the study is completed (at which time they will be destroyed). If you desire access to the recordings, you will not have access to the recordings until the end of participation in the study, at which time you could make arrangements to listen to them.

Whereas information you disclose in this study will remain completely confidential, there are five specific cases in which we might need to break confidentiality, per the College of Psychologists of Ontario regulations: (1) If you intend to harm yourself; (2) if you intend on harming someone else; (3) If there is reasonable suspicion that a child up to the age of 16 years is at risk of neglect or abuse, we are required by law to report this to the Children's Aid Society right away; (4) if our files are subpoenaed by the courts (records can be opened by a specific court order); and (5) if a regulated health professional (such as a psychologist) has engaged in inappropriate (e.g., abusive) behaviour toward you and you provide us with the name of this individual, we are obligated to report them to their regulatory body (e.g., the College of Psychologists of Ontario).

\section{Incentives to Participate:}

You will not receive any financial compensation or incentives to participate in this study. If eligible, you will receive an effective psychological treatment for insomnia, provided by supervised therapists.

\section{Costs and/or Compensation for Participation:}

No other costs associated with participation (e.g., office visits, transportation, etc.) will be compensated. 


\title{
Voluntary Nature of Participation:
}

Participation in this study is voluntary. Your choice of whether or not to participate will not influence your future relations with Ryerson University. If you decide to participate, you are free to withdraw your consent and to stop your participation at any time without penalty or loss of benefits to which you are allowed. At any particular point in the study, you may refuse to answer any particular question or stop participation altogether.

\section{Questions about the Study:}

If you have any questions about the research now, please ask. If you have questions later about the research, you may contact.

Principal Investigator: Colleen Carney, Ph.D.

Telephone Number: (416) 979-5000 ext. 2177.

If you have questions regarding your rights as a human subject and participant in this study, you may contact the Ryerson University Research Ethics Board for information.

\author{
Toni Fletcher \\ c/o Office of the Vice President, Research and Innovation \\ Ryerson University \\ 350 Victoria Street \\ Toronto, ON M5B 2K3 \\ 416-979-5000 Ext. 7112
}

\section{Agreement:}

Your signature below indicates that you have read the information in this agreement and have had a chance to ask any questions you have about the study. Your signature also indicates that you agree to be in the study and have been told that you can change your mind and withdraw your consent to participate at any time. Additionally, your signature indicates that you agree to have your therapy sessions audiotaped. You have been given a copy of this agreement.

You have been told that by signing this consent agreement you are not giving up any of your legal rights.

\footnotetext{
Name of Participant (please print)
}

Signature of Participant

Date

Signature of Investigator

Date 


\section{Appendix F}

\section{Ryerson University \\ Consent Agreement}

\section{Activity, Fatigue and Sleep Among Healthy Sleepers}

You are being asked to participate in a research study. Before you give your consent to be a volunteer, it is important that you read the following information and ask as many questions as necessary to be sure you understand what you will be asked to do.

\section{Investigators:}

Principal Investigator, Colleen E. Carney, Ph.D., Ryerson University Co-Investigators, Taryn G. Atlin, M.A.,\& Andrea L. Harris, M.A., Ryerson University

\section{Purpose of the Study:}

The purpose of this study is to examine the relationship between activity and sleep. Approximately 100 males and females (70 people in the final sample) between 18-74 years old, will be enrolled in this study. We will enroll those who are otherwise physically and emotionally healthy, and without any conditions that would interfere with their safe participation.

\section{Description of the Study:}

If you agree to be in this study, you will be asked to sign this consent form. You will be asked to participate in a screening interview that consists of a structured sleep and psychiatric interview at the Ryerson Sleep and Depression Laboratory to ensure that you are eligible. If you qualify for the study based on the results of the screening interview, you will be asked to take part in a number of optional study procedures and visits as outlined below:

Visit \#1 (2 hours)

- Screening interview (structured sleep and psychiatric interview)

- If eligible based on the screening interview, you will be asked to participate in a 15minute task, whereby you will be instructed to imagine the feeling of fatigue and answer some questions.

- You will also be asked to do some self-monitoring tasks over the next two weeks:

- Completing a sleep log each morning in your home (about 5 minutes) for two weeks to answer questions about the previous night's sleep.

- Completing an activity log each evening in your home (about 5 minutes) for two weeks to answer questions about your daytime activities.

- Wearing a small wristwatch-sized device called an actigraph for one week to measure your sleep at night and rate your fatigue three times per day when the alarm on the device sounds. You will be taught how to use this device by the study staff and will be asked to wear it at all times (even when you sleep), with the exception of the times when you are bathing or when you believe your wrist would be exposed to water.

- You will be asked to allow a study staff to measure your waist circumference, as well as your height and weight in order to measure body mass index. 
- You will be asked to complete questionnaires, which will ask about your mood and sleep.

Visit \#2 (5 minutes)

- You will be asked to return your monitoring diary and the watch to the Ryerson Sleep and Depression Laboratory.

\section{What is Experimental in this Study:}

None of the procedures or questionnaires used in this study are experimental in nature.

\section{Risks or Discomforts:}

You may experience some anxiety and discomfort while completing the questionnaires, as some of the questions we will ask you as part of this study may make you feel uncomfortable. However, the questions are not personal and refer to symptoms only. In addition, you may experience embarrassment or discomfort when height, weight, and waist circumference are measured by study staff to assess for the proxy parameters of metabolic syndrome. In order to manage potential discomfort, you will be given several options for this procedure. You do not have to bare your skin and you can choose to gather these measurements yourselfby following study staffs' instructions. You may also choose not to engage in these procedures at any time without any penalty. Although these risks are minimal, you are free to withdraw from participation at any time if this becomes too distressing.

You may refuse to answer any of the questions and you may take a break at any time during the study. You may stop your participation in this study at any time. You may find it uncomfortable to wear the wristwatch device, if normally you do not wear a wristwatch. Wearing the wristwatch device is voluntary.

\section{Benefits of the Study:}

Understating healthy sleep helps us to understand what causes poor sleep. The anticipated benefit of this study is that the information learned from this study will provide us with information pertaining to activity and sleep, such that we can refine current sleep treatments for those with insomnia. We cannot guarantee direct benefits for participants.

\section{Confidentiality:}

Except when required by law, you will not be identified by name, address, telephone number, or any other direct personal identifier in study records disclosed outside of Ryerson University. Your data will be kept in a locked file in the Project Coordinator's office at Ryerson University. While the information and data resulting from this study may be presented at scientific meetings or published in a scientific journal, your identity will not be revealed.

Your study contains a unique code and this code will be used so that no identifying information will be tied to your study information. We will maintain some identifying information in a password-protected database on the Project Coordinator's computer in a locked office. This separate database will contain your name and contact information only. Only study related personnel have access to your personal information. They will have access to your name for the 
purposes of addressing you personally. Files will be stored to the extent allowed by law for 10 years after the study's completion.

While we will take the above measures to protect your personal identification, there are some limits to confidentiality which are as follows:

1. If you tell us you are a danger to yourself or someone else, we will be required to break confidentiality to keep you safe

2. If you tell us that you know of a child who is being abused or is at risk of being abused, we will contact Children's Aid Society

3. If you tell us that a regulated health professional (e.g., physician, nurse, dentist) was sexually inappropriate with you, we will be required to notify that persons' regulatory body.

4. If a court of law requests to subpoena your records, we would be required to do so.

\section{Incentives to Participate:}

You will be contributing to scientific knowledge by providing information on the health benefits of daily activities on sleep.

\section{Costs and/or Compensation for Participation:}

You will receive financial compensation to participate in this study. This will include up to \$20 for visit 1 and, if eligible, $\$ 20$ for the at home monitoring. If you complete all study components, you would receive a total of $\$ 40$ at visit 2 . If you are not eligible for complete study participation, you will receive $\$ 10$ for your participation in the diagnostic interview. No other costs associated with participation (e.g., transportation, etc.) will be compensated.

\section{Voluntary Nature of Participation:}

Participation in this study is voluntary. Your choice of whether or not to participate will not influence your future relations with Ryerson University. If you decide to participate, you are free to withdraw your consent and to stop your participation at any time without penalty or loss of benefits to which you are allowed. At any particular point in the study, you may refuse to answer any particular question or stop participation altogether.

\section{Questions about the Study:}

If you have any questions about the research now, please ask. If you have questions later about the research, you may contact.

$$
\begin{aligned}
& \text { Principal Investigator: Colleen Carney, Ph.D. } \\
& \text { Telephone Number: (416) 979-5000 ext. } 2177 .
\end{aligned}
$$

If you have questions regarding your rights as a human subject and participant in this study, you may contact the Ryerson University Research Ethics Board for information.

Toni Fletcher

c/o Office of the Vice President, Research and Innovation

Ryerson University

350 Victoria Street

Toronto, ON M5B 2K3 


\section{Agreement:}

Your signature below indicates that you have read the information in this agreement and have had a chance to ask any questions you have about the study. Your signature also indicates that you agree to be in the study and have been told that you can change your mind and withdraw your consent to participate at any time. Additionally, your signature indicates that you agree to have your therapy sessions audiotaped. You have been given a copy of this agreement.

You have been told that by signing this consent agreement you are not giving up any of your legal rights.

Name of Participant (please print)

Signature of Participant

Signature of Investigator
Date

Date 


\section{References}

Aaronson, L. S., Pallikkathayil, L., \& Crighton, F. (2003). A qualitative investigation of fatigue among healthy working adults. Western Journal of Nursing Research, 25, 419-33.

Aaronson, L. S., Teel, C. S., Cassmeyer, V., Neuberger, G. B., Pallikkathayil, L., Pierce, J., ... Wingate, A. (1999). Defining and measuring fatigue. Journal of Nursing Scholarship, 31, 45-50.

Alapin, I., Fichten, C. S., Libman, E., Creti, L., Bailes, S., \& Wright, J. (2000). How is good and poor sleep in older adults and college students related to daytime sleepiness, fatigue, and ability to concentrate? Journal of Psychosomatic Research, 49, 381-390.

American Academy of Sleep Medicine. (2005). International classification of sleep disorders. Diagnostic and Coding Manual ( $2^{\text {nd }}$ ed.). Westchester, Illinois: Author.

American Psychiatric Association (2013). Diagnostic and statistical manual of mental disorders, ( $5^{\text {th }}$ ed.). Washington, DC: Author.

American Sleep Disorders Association. (1997). The international classification of sleep disorders (revised). Rochester, MN: Author.

Ancoli-Israel, S., Cole, R., Alessi, C., Chambers, M., Moocroft, W., \& Pollak, C. P. (2003). The role of actigraphy in the study of sleep and circadian rhythms. Sleep, 26, $342-392$.

Ancoli-Israel, S., \& Roth, T. (1999). Characteristics of insomnia in the United States: Results of the 1991 National Sleep Foundation Survey I. Sleep, 22, S347-S353.

Antony, M. M., Bieling, P. J., Cox, B. J., Enns, M. W., \& Swinson, R. P. (1998). Psychometric properties of the 42-item and 21-item versions of the depression anxiety stress scales in clinical groups and a community sample. Psychological Assessment, 10, 176 - 181. 
Armutlu, K., Cetisli, K. N., Keser, I, Sumbuloglu, V., Irem, D. A., Zafer, G., \& Karabudak, R. (2007). The validity and reliability of the fatigue severity scale in Turkish multiple sclerosis patients. International Journal of Rehabilitation Research, 30, 81 - 85.

Backhaus, J., Junghanns, K., \& Hohagen, F. (2004). Sleep disturbances are correlated with decreased morning awakening salivary cortisol. Psychoneuroendocrinology, 29, 1184 1191.

Baron, R. M., \& Kenny, D. A. (1986). The moderator-mediator variable distinction in social psychological research: Conceptual, strategic, and statistical considerations. Journal of Personality and Social Psychology, 51, 1173-1182.

Bastien, C. H., Vallières, A. \& Morin, C. M. (2001). Validation of the insomnia severity index as an outcome measure for insomnia research. Sleep Medicine, 2, $297-307$.

Bonnet, M. H, \& Arand, D. L. (1995). 24-hour metabolic rate in insomniacs and matched normal sleepers. Sleep, 18, 581-8.

Bonnet, M. H, \& Arand, D. L. (1996). The consequences of a week of insomnia. Sleep, 19, 453 461.

Bonnet, M. H, \& Arand, D. L. (1998). The consequences of a week of insomnia II: Patients with insomnia. Sleep, 21, $359-368$.

Borkovec, T. D., Ray, W. J., \& Stöber, J. (1998). Worry: A cognitive phenomenon intimately linked to affective, physiological, and interpersonal behavioral processes. Cognitive Therapy and Research, 22, 561-576.

Bootzin, R. R. (1972). A stimulus control treatment for insomnia. Proceedings of the American Psychological Association, 7, 395 - 396. 
Broman, J. E., \& Hetta, J. (1994). Perceived pre-sleep arousal in patients with persistent psychophysiologic and psychiatric insomnia. Nordic Journal of Psychiatry, 48, 203 207.

Broman, J. E., Lundh, L. G., Aleman K, \& Hetta, J. (1992). Subjective and objective performance in patients with persistent insomnia. Scandinavian Journal of Behavior Therapy, 21, 115-126.

Bryk, A. S. \& Raudenbush, S. W. (1992). Hierarchical linear models: Applications and data analysis methods. Newbury Park, CA: Sage.

Buysse, D. J., Ancoli-Israel, S., Edinger, J. D., Lichstein, K. L., \& Morin, C. M. (2006). Recommendations for a standard research assessment of insomnia. Sleep, 29, $1155-$ 1173.

Buysse, D. J., Reynolds, C. F., Monk, T.H., Hoch, C.C., Yeager, A.L., \& Kupfer, D.J. (1991). Quantification of subjective sleep quality in healthy elderly men and women using the Pittsburgh Sleep Quality Index (PSQI). Association of Professional Sleep Societies, 14, $331-338$.

Buysse, D. J., Wesley, T., Scott, J., Franzen, P. L., Germain, A., Hall, M., ... Kupfer, D. J. (2007). Daytime symptoms in primary insomnia: A prospective analysis using ecological momentary assessment. Sleep Medicine, 8, 198 - 208.

Buysse, D. B., Cheng, Y., Germain, A., Moul, D. E., Franzen, P. L., Fletcher, M. \& Monk, T. H. (2010). Night-to-night sleep variability in older adults with and without chronic insomnia. Sleep Medicine, 11, 56 - 64.

Carey, T. J., Moul, D. E., Pilkonis, P., Germain, A., \& Buysse, D. J. (2005). Focusing on the experience of insomnia. Behavioural Sleep Medicine, 3, 73 - 86. 
Carney, C. E., Buysse, D. J., Ancoli-Israel, S., Edinger, J. D., Krystal, A. D., Lichstein, K. L., \& Morin, C. M. (2012). The consensus sleep diary: Standardizing prospective sleep selfmonitoring. Sleep, 35, $287-302$.

Carney, C. E., \& Edinger, J. D. (2006). Identifying critical dysfunctional beliefs about sleep in primary insomnia. Sleep, 29, 325-333.

Carney, C. E., Edinger, J. D., Meyer, B., Lindman, L., \& Istre, T. (2006). Symptom-focused rumination and sleep disturbance. Behaviour Sleep Medicine, 4, 228-241

Carney, C. E., Edinger, J. E., Morin, C. M., Manber, R., Rybarczyk, B., Stepanski, E., Wright, H., \& Lack, L. (2010). Examining maladaptive beliefs about sleep across insomnia patient groups. Journal of Psychosomatic Research, 68, 57 - 65.

Carney, C. E, Harris, A. L., Falco, A., \& Edinger, J. D. (2013). The relation between insomnia symptoms, mood and rumination about insomnia symptoms. Journal of Clinical Sleep Medicine, 9, $567-575$.

Carney, C. E., Harris, A. L., Moss, T. G., \& Edinger, J. D. (2010). Distinguishing rumination from worry in clinical insomnia. Behaviour Research and Therapy, 48, 540 - 546.

Chambers, M. J., \& Keller, B. (1993). Alert insomniacs: Are they really sleep deprived? Clinical Psychology Reviews, 13, 649-666.

Chambless, D. L., Caputo, G. C., Jasin, S. E., Gracely, E. J., \& Williams, C. (1985). The mobility inventory for agoraphobia. Behaviour Research and Therapy, 23, 35-44.

Chaudhuri, A. \& Behan, P. O. (2000). Fatigue and basal ganglia. Journal of Neurological Sciences, 179, $34-42$.

Chaudhuri, A. \& Behan, P. O. (2004). Fatigue in neurological disorders. Lancet, 363, 978 - 988. 
Chesson, A. L., Littner, M., Davila, D., Anderson, W. M., Grigg-Damberger, M., Hartse, K., Johnson, S., \& Wise, M. (1999). Practice parameters for the use of light therapy in the treatment of sleep disorders. Sleep, 22, $641-660$.

Chesson, A., Hartse, K., Anderson, M., Davila, D., Johnson, S., Littner, M. Wise, M., \& Rafecas, J. (2000). Practice parameters for the evaluation of chronic insomnia. Sleep, 23, $1-5$.

Coates, T. J., George, J. M., Killen, J. D., Marchini, E., Hamilton, S., Thorensen, C. E. (1981). First night effects in good sleepers and sleep-maintenance insomniacs when recorded at home. Sleep, 4, 293-298.

Collins, L. M., Schafer, J. L., \& Kam, C. M. (2001). A comparison of inclusive and restrictive strategies in modern missing data procedures. Psychological Methods, 6, $330-351$.

Daley, M., Morin, C. M., LeBlanc, M., Gregoire, J. P., \& Savaard, J. (2009). Economic burden of insomnia: Direct and indirect costs for individuals with insomnia syndrome, insomnia symptoms and good sleepers. Sleep, 32, $55-64$.

Dzierzewski, J. M., Williams, J. M., Roditi, D., Marsiske, M., McCoy, K., McNamara, J., ... McCrae, C. S. (2010). Daily variations in objective nighttime sleep and subjective morning pain in older adults with insomnia: Evidence of covariation over time. Journal of American Geriatric Society, 58, 925 - 930.

Edinger, J. D., Bonnet, M., Bootzin, R. R., Doghramji, K., Dorsey, C. M., Espie, C. A., ... Stepanski, E., J. (2004). Derivation of research diagnostic criteria for insomnia: Report on an American Academy of Sleep Medicine work group. Sleep, 27, 1567-1596.

Edinger, J. D., Fins, A. I., Sullivan, R. J. Jr., Marsh, G. R., Dailey, D. S., Hope, T. V., ... Vasilas. D. (1997). Sleep in the laboratory and sleep at home: comparisons of older insomniacs and normal sleepers. Sleep, 20, 1119-1126. 
Edinger, J., Kirby, A., Lineberger, M., Loiselle, M., Wohlgemuth, W., \& Means, M. (2004). The Duke Structured Interview for Sleep Disorders. Duke University Medical Center.

Edinger, J. D., Marsh, G. R., Mccall, W. V., Erwin, C. W., Lininger, A. W. (1991). Sleep variability across consecutive nights of home monitoring in older mixed dims patients. Sleep, 14, 13-17.

Edinger, J. D., Means, M. K., Carney, C. E., \& Krystal, A. D. (2008). Psychomotor performance deficits and their relation to prior nights' sleep among individuals with primary insomnia. Sleep, 31, 599-607.

Edinger, J. D., Means, M. K., Stechuchak, K. M., \& Olsen, M. K. (2004). A pilot study of inexpensive sleep-assessment devices. Behavioural Sleep Medicine, 2, 41 - 49.

Edinger, J. D., \& Wohlgemuth, W. (1999). The significance and management of persistent primary insomnia: The past, present and future of behavioural insomnia therapies. Sleep Medicine Review, 3, $101-118$.

Edinger, J. D., Wohlgemuth, W. K., Radtke, R. A., Marsh, G. R. \& Quillian, R. E. (2001). Cognitive behavioral therapy for treatment of chronic primary insomnia: A randomized controlled trial. JAMA, 11, $1856-1864$.

Espie, C. A. (2002). Insomnia: Conceptual issues in the development, persistence, and treatment of sleep disorders in adults. Annual Review Psychology, 53, 215 - 243.

Espie, C. A., Broomfield, N. M., MacMahon, K. M., Macphee, L.M., \& Taylor, L. M. (2006). The attention-intention-effort pathway in the development of psychophysiologic insomnia: A theoretical review. Sleep Medicine Reviews, 10, 215 - 245. 
Feinberg, I., March, J. D., Floyd, T. C., Jimison, R., Bossom-Demitrack, L., \& Katz, P. H. (1985). Homeostatic changes during post-nap sleep maintain baseline levels of delta EEG. Electroencephalography and Clinical Neuropshysiology, 61, 134 - 137.

Ferentinos, P., Kontaxakis, V., Havaki-Kontaxaki, B., Dikeos, D., \& Lykouras, L. (2011). Psychometric evaluation of the fatigue severity scale in patients with major depression. Quality of Life Research, 20, $457-465$.

Fichten, C.S., Libman, E., Bailes, S., \& Alapin, I. (2000). Characteristics of older adults with insomnia. In K.L. Lichstein \& C.M. Morin (Eds.), Treatment of late life insomnia (pp. 37-80). New York: Sage.

Fichten, C. S., Libman, E., Creti, L., Amsel, R., Tagalakis, V., \& Brender, W. (1998). A cognitive model of insomnia. Proceedings / Actes du colloque de l'Association pour la rescherche au collegial (ARC), 9, $136-140$.

Fichten, C. S., Creti, L., Amsel, R., Brender, W., Weinstein, N., \& Libman, E. (1995). Poor sleepers who do not complain of insomnia: Myths and realities about psychological and lifestyle characteristics of older good and poor sleepers. Journal of Behaviour Medicine, 18, 189-223.

First, M. B., Spitzer, R. L., Gibbon, M., \& Williams, J. B. (2002). Structured Clinical Interview for DSM-IV-TR Axis I Disorders, Research Version, Patient Edition (SCID-I/P). New York: Biometrics Research, New York State Psychiatric Institute.

Ford, D. E., \& Kamerow, C. A. (1989). Epidemiologic study of sleep disturbances and psychiatric disorders. An opportunity for prevention? Journal of the American Medical Association, 262, $1479-1484$. 
Fortier-Brochu, E., Beaulieu-Bonneau, S., Ivers, H., \& Morin, C. M. (2010). Relations between sleep, fatigue, and health-related quality of life in individuals with insomnia. Journal of Psychosomatic Research, 69, $475-483$.

Hale, L., Do, D. P., Basurto-Davila, R., Heron, M., Finch, B. K., Dubowitz, T., Lurie, N., \& Bird, C. E. (2009). Does mental health history explain gender disparities in insomnia symptoms among young adults? Sleep Medicine, 10, 1118 - 1123.

Hall, M., Buysse, D. J., Reynolds, C. F., Kupfer, D. J., Baum, A. (1996). Stress related intrusive thoughts disrupt sleep onset and continuity. Sleep Research, 25, 163.

Harris, A. L. \& Carney, C. E. (2012). Can we modify maladaptive attributions for fatigue? Cognitive Behavioural Therapy, 41, $40-50$.

Harris, A. L., \& Carney, C. E. (2009, November). Are people with insomnia afraid of fatigue? Poster presented at the annual meeting of the Association of Behavioral and Cognitive Therapies, New York, NY.

Harris, A. L., Carney, C. E., \& Moss, T. G. (2010). Towards insomnia-specific models and assessment of rumination. Sleep, 33, A210.

Harvey, A. (2002). A cognitive model of insomnia. Behavior Research and Therapy, 40, $869-$ 893.

Hathaway, S. R. \& McKinley, J. C. (1967). The Minnesota Multiphasic Personality Inventory Manual. New York: Psychological Corporation.

Hayashi, M., Watanabe, M., \& Hori, T. (1999). The effects of a 20 minute nap in the midafternoon on mood, performance and EEG activity. Clinical Neurophysiology, 110, 272 279. 
Hayes, A. F. (2008). Beyond Baron and Kenny: Statistical mediation analysis in the new millennium. Communication Monographs, 76, 408 - 420.

Haynes, S. N., Adams, A. E., \& Franzen, M. (1981). The effects of presleep stress on sleep-onset insomnia. Journal of Abnormal Psychology, 90, $601-606$.

Hauri, P. Fisher, J. (1986). Persistent psychophysiologic (learned) insomnia. Sleep 9, 38-53.

Hauri, P. J., \& Wisbey, J. (1992). Wrist actigraphy in insomnia. Sleep, 15, 293 - 301.

Healy, E. S., Kales, A., Monroe, L. J., Bixler , E. O., Chamberlin, K., \& Soldatos. C. R. (1981). Onset of insomnia: Role of life-stress events. Psychosomatic Medicine, 43, 439-451.

Hoch, C. C., Dew, M. A., Reynolds, C. F., Monk, T. H., Buysse, D. J., Houck, P. R., ... Kupfer, D. J. (1994). A longitudinal study of laboratory- and diary-based sleep measures in healthy "old old" and "young old" volunteers. Sleep, 17, 489-496.

Hossain, J. L., Ahmad, P., Reinish, L. K., Hossain, N. K., \& Shapiro, C. M. (2005). Subjective fatigue and subjective sleepiness: Two independent consequences of sleep disorders? Journal of Sleep Research, 14, 245.

Hultsch, D. F., MacDonald, S. W. S., Hunter, M. A., Levy-Bencheton, J., \& Strauss, E. (2000). Intraindividual variability in cognitive performance in older adults: Comparison of adults with mild dementia, adults with arthritis, and healthy adults. Neuropsychology, 14, 588598.

Johns, M. W. (1991). A new method for measuring daytime sleepiness: The Epworth Sleepiness Scale. Sleep, 14, 540-545.

Jacobs, G. D. Pace-Schott, E. F., Stickgold, R. \& Otto, M. W. (2004). Cognitive therapy and pharmacotherapy for insomnia: A randomized controlled trial and direct comparison. Archives of Internal Medicine, 164, 1888 - 1896. 
Kales, A., Caldwell, A. B., Soldatos, C. R., Bixler, E. O., \& Kales, J. D. (1983).

Biopsychobehavioral correlates of insomnia II. Pattern specificity and consistency with the Minnesota multiphasic personality inventory. Psychosomatic Medicine, 45, $341-$ 356.

Kales, J. D., Kales, A., Bixler, E. O., Soldatos, C. R., Cadieux, R. J., Kashurba, G. J., \&VelaBueno, A. (1984). Biopsychobehavioral correlates of insomnia, V: clinical characteristics and behavioral correlates. American Journal of Psychiatry, 141, 1371-1376.

Katz, D. A. \& McHorney, C. A. (1998). Clinical correlates of insomnia in patients with chronic illness. Archives of Internal Medicine, 158, 1099 - 1107.

Kay, D. B., Dzierzewski, J. M., Rowe, M. \& McCrae, C. S. (2013). Greater night-to-night variability in sleep discrepancy among older adults with a sleep complaint compared to noncomplaining older adults. Behavioral Sleep Medicine, 11, 72 - 90.

Kloss, J. D. (2003). Daytime sequelae of insomnia. In M. P. Szuba, J. D. Kloss, \& D. F. Dinges (Eds.), Insomnia: Principles and management (pp. 23-42). Cambridge: Cambridge University Press.

Knutson, K.L., Rathouz, P.J., Yan, L.L., Liu, K., Lauderdale, D.S. (2007). Intra-individual daily and yearly variability in actigraphically recorded sleep measures: the CARDIA study. Sleep 30, 793-796.

Kohn, L., \& Espie, C. A. (2005). Sensitivity and specificity of the insomnia experience: A comparative study of psychophysiological insomnia, insomnia associated with a mental disorder and good sleep. Sleep, 28, $104-112$. 
Kroenke, K., Wood, D. R., Manglesdorff, A. D., Meier, N. J., \& Powell, J. B. (1988). Chronic fatigue in primary care. Prevalence, patient characteristics, and outcome. JAMA, 260, $929-34$.

Krupp, L. B., LaRocca, N. G., Muir-Nash, J., \& Steinberg, A. D. (1989). The fatigue severity scale. Application to patients with multiple sclerosis and systemic lupus erythematosus. Archives of Neurology, 46, 1121-1123.

Leger, D., Guilleminault, C., Bader, G., Levy, E., \& Paillard, M. (2002). Medical and socioprofessional impact of insomnia, Sleep, 25, $625-629$.

Leproult, R., Copinschi, G., Buxton, O., van Cauter, E. (1997). Sleep loss results in an elevation of cortisol levels the next evening. Sleep, 20, $865-870$.

Lerdal, A., Wahl, A. K., Rustoen, T., Hanestad, B. R., \& Moum, T. (2005). Fatigue in the general population: A translation and test of the psychometric properties of the Norwegian version of the fatigue severity scale. Scandinavian Journal of Public Health, $33,123-130$.

Lichstein, K. L. \& Johnson, R. S. (1994). Pupollometric discrimination of insomniacs. Behaviour Research and Therapy, 32, $123-129$.

Lichstein, K. L., Means, M. K., Noe, S. L., \& Aguillard, R. N. (1997). Fatigue and sleep disorders. Behaviour Research and Therapy, 35, 733-740.

Lichstein, K. L., Riedel, B. W., Wilson, N. M., Lester, K. W., \& Aguillard, R. N. (2001). Relaxation and sleep compression for late-life insomnia: A placebo-controlled trial. Journal of Consulting and Clinical Psychology, 69, 227-239.

Lichstein, K. L. \& Rosenthal, T. L. (1980). Insomniacs’ perceptions of cognitive versus somatic determinants of sleep disturbance. Journal of Abnormal Psychology, 89, 105 - 107. 
Lichstein, K. L., Wilson, N. M., Noe, S., Aguillard, R. N., \& Bellur, S. N. (1994). Daytime sleepiness in insomnia: Behavioural, biological, and subjective indices. Sleep, 17, 693702.

Lobbestael, J., Leurgans, M., \& Arntz, A. (2011). Inter-rater reliability of the structured clinical interview for DSM-IV Axis I Disorders (SCID I) and Axis II Disorders (SCID II). Clinical Psychology and Psychotherapy, 18, $75-79$.

Lovibond, S. H., \& Lovibond, P. F. (1995). Manual for the Depression Anxiety Stress Scales, (2nd ed.). Sydney, Australia: Psychology Foundation of Australia.

Lundh, L. \& Broman, J. (2000). Insomnia as an interaction between sleep-interfering and sleepinterpreting processes. Journal of Psychosomatic Research, 49, 299 - 310.

MacKinnon, D. P., Lockwood, C. M., \& Williams, J. (2004). Confidence limits for the indirect effect: Distribution of the product and resampling methods. Multivariate Behavioral Research, 39, 99-128.

Maxwell, S. E. \& Cole, D. A. (2007). Bias in cross-sectional analyses of longitudinal mediation. Psychological Methods, 12, $23-44$.

McCall, C. \& McCall, V. (2012). Comparison of actigraphy with polysomnography and sleep logs in depressed insomniacs. Journal of Sleep Research, 21, $122-127$.

McCrae, C. S., McNamara, J. P., Rowe, M. A., Dzierzewski, J. M., Dirk, J., Marsiske, M. \& Craggs, J. G. (2008). Sleep and affect in older adults: Using multilevel modeling to examine daily associations. Journal of Sleep Research, 17, 42 - 53.

McCrae, C. S., Rowe, M. A., Tierney, C. G., Dautovich, N. D., DeFinis, A. L., McNamara, J. P. H. (2005). Sleep complaints, subjective and objective sleep patterns, health, 
psychological adjustment, and daytime functioning in community-dwelling older adults. The Journals of Gerontology: Psychological Sciences, 60B, 182-189.

McCrae, C. S., Wilson, N. M., Lichstein, K. L., Durrence, H. H., Taylor, D. J., Bush, A. J., Riedel, B. W. (2003). 'Young old' and 'old old' poor sleepers with and without insomnia complaints. Journal of Psychosomatic Research, 54, 11-19.

Means, M. K., Edinger, J. D., Glenn, D. M. \& Fins, A. I. (2003). Accuracy of sleep perceptions among insomnia sufferers and normal sleepers. Sleep Medicine, 4, 285 - 296.

Means, M. K., Lichstein, K. L., Epperson, M. T., Johnson, C. T. (2000). Relaxation therapy for insomnia: Nighttime and day time effects. Behaviour Research Therapy, 38, $665-678$.

Mezick, E. J., Matthews, K. A., Hall, M., Kamarack, T. W., Buysse, D. J., Owens, J. F., \& Reis, S. E. (2009). Intra-individual variability in sleep duration and fragmentation: Association with stress. Psychoneuroendocrinology, 2009, $1346-1354$.

Monroe, L. J. (1967). Psychological and physiological differences between good and poor sleepers. Journal of Abnormal Psychology, 72, 255-264.

Morgan, K., \& Clarke, D. (1997). Risk factors for late-life insomnia in a representative general practice sample. British Journal of General Practice. 47, 166 - 169.

Morgan, K. (2003). Daytime activity and risk factors for late life insomnia. Journal of Sleep Research, 12, 231 - 238.

Morin, C. M. (1993). Insomnia: Psychological assessment and management. New York: Guilford.

Morin, C. M., Bootzin, R. R., Buysse, D. J., Edinger, J. D., Espie, C. A., Lichstein, K. L. (2006). Psychological and behavioral treatment of insomnia: Update of the recent evidence (1998 -2004). Sleep, 29, $1398-1413$. 
Morin, C. M., LeBlanc, M., Daley, M., Gregoirs, J. P., \& Merette, C. (2006). Epidemiology of insomnia: Prevalence, self-help treatments, consultations, and determinants of helpseeking behavior. Sleep Medicine, 7, 123 - 130.

Morin, C. M., Stone, J., Trinkle, D., Mercer, J., \& Remsberg, S. (1993). Dysfunctional beliefs and attitudes about sleep among older adults with and without insomnia complaints. Psychology and Aging, 8, $463-467$.

Morin, C. M., Vallières, A., \& Ivers, H. (2007). Dysfunctional beliefs and attitudes about sleep (DBAS): Validation of a brief version (DBAS-16). Sleep, 30, 1547 - 1554.

Moul, D. E., Nofzinger, E. A., Pilkonis, P. A., Houck, P. R., Miewald, J. M., \& Buysse, D. J. (2002). Symptom reports in severe chronic insomnia. Sleep, 25, $553-563$.

Moul, D. E., Hall, M., Pilkonis, P. A., Buysse, D. J. (2004). Self-report measures of insomnia adults: Rationales, choices, and needs. Sleep Medicine Reviews, 8, 177-198

Muller, D., Judd., C. M., \& Yzerbyt, V. Y. (2005). When moderation is mediated and mediation is moderated. Journal of Personality and Social Psychology, 89, $852-863$.

Murtagh, D. R. \& Greenwood, K. M. (1995). Identifying effective psychological treatments for insomnia: A meta-analysis. Journal of Consulting and Clinical Psychology, 63, 79 - 89. National Institutes of Health State-of-the-Science Conference Statement on Manifestations and Management of Chronic Insomnia in Adults. (2005). NIH Consens Sci Statements, 2, 130.

Neitzert-Semler, C., \& Harvey, A. G. (2007). An experimental investigation of daytime monitoring for sleep-related threat in primary insomnia. Cognition \& Emotion, 21, 146 161. 
Nolen-Hoeksema, S. (1991). Responses to depression and their effects on the duration of depressive episodes. Journal of Abnormal Psychology, 100, 569-582.

O’Brien, E. M., Waxenberg, L. B., Atchison, J. W., Gremillion, H. A., Staud, R. M., McCrae, C. S., \& Robinson, M. E. (2011). Intraindividual variability in daily sleep and pain ratings among chronic pain patients: Bidirectional association and the role of negative mood. Clinical Journal of Pain, 27, $425-433$.

Ohayon, M. M. (2002) Epidemiology of insomnia: What we know and what we still need to learn. Sleep Medicine Review, 6, 97 - 111.

Ohayon, M. M., Carskadon, M. A., Guilleminault, C., \& Vitello, M. D. (2004). Meta-analysis of quantitative sleep parameters from childhood to old age in healthy individuals: developing normative sleep values across the human lifespan. Sleep, 27, 1255-1273.

Ohayon, M. M., Caulet, M., Lemoine, P. (1998). Comorbidity of mental and insomnia disorders in the general population. Comprehensive Psychiatry, 39, 185 - 197.

Ozminkowski, R. J., Wang, S. \& Walsh, J. K. (2007). The direct and indirect costs of untreated insomnia in adults in the United States. Sleep, 30, $263-273$.

Pedhazur, E. J., (1997). Multiple Regression in Behavioral Research (3rd ed.). Orlando, FL: Harcourt Brace.

Petruzzello, S. J., Hall, E. E., \& Ekkekakis, P. (2001). Regional brain activation as a biological marker of affective responsivity to acute exercise: Influence of fitness. Psychophysiology, $38,99-106$.

Pigeon, W. R., \& Perlis, M. L. (2006). Sleep homeostasis in primary insomnia. Sleep Medicine Reviews, 10, $247-254$. 
Pigeon, W. R., Sateia, M. J., \& Furgeson, R. J. (2003). Distinguishing between excessive daytime sleepiness and fatigue: Toward improved detection and treatment. Journal of Psychosomatic Research, 54, $61-69$.

Preacher, K. J., \& Hayes, A. F. (2008). Asymptotic and resampling strategies for assessing and comparing indirect effects in multiple mediator models. Behavior Research Methods, 40, $879-891$.

Puetz, T. W., O’Connor, P. J., \& Dishman, R. K. (2006). Effects of chronic exercise on feelings of energy and fatigue: A quantitative synthesis. Psychological Bulletin, 132, 866-876.

Raudenbush, S. W. \& Bryk, A. S. (2002). Hierarchical linear models: Applications and data analysis methods $\left(2^{\text {nd }}\right.$ ed). Thousand Oaks, CA: Sage.

Ree, M.J., \& Harvey, A.G. (2004). Insomnia. In J. Bennett-Levy G. Butler, M. Fennell, A. Hackmann, M. Mueller, and D. Westbrook (Eds.). The Oxford Handbook of Behavioural Experiments in Cognitive Therapy (pp.287-305). Oxford: Oxford University Press.

Riedel, B. W. \& Lichstein, K. L. (2000). Insomnia and daytime functioning. Sleep Medicine Reviews, 4, $277-298$.

Riemann, D., Spiegelhalder, K., Feige, B., Voderholzer, U., Berger, M., Perlis, M., \& Nissen, C. (2010). The hyperarousal model of insomnia: A review of the concept and its evidence. Sleep Medicine Reviews, 14, 19 - 31.

Roth, T. (2007). Insomnia: Definition, prevalence, etiology, and consequences. Journal of Clinical Sleep Medicine, 3, S7 - S10.

Roth, T. \& Ancoli-Israel, S. (1999). Daytime consequences and correlates of insomnia in the United States: Results of the 1991 National Sleep Foundation Survey II. Sleep, 22, S354 $-\mathrm{S} 358$. 
Rowe, M., McCrae, C., Campbell, J., Horne, C., Tiegs, T., Lehman, B., \& Cheng, J. (2008). Actigraphy in older adults: Comparison of means and variability of three different aggregates of measurement, Behavioural Sleep Medicine, 6, 127 - 145.

Rubin, D. B. (1976). Inference and missing data. Biometrika, 63, 581 - 592.

Sadeh, A., \& Acedbo, C. (2002). The role of actigraphy in sleep medicine. Sleep Medicine Reviews, 6, 113-124.

Sanchez-Ortuno, M. M., Carney, C. E., Edinger, J. D., \& Harris, A. (2011). Moving beyond average values: Assessing the night-to-night instability of sleep and arousal in DSM-IVTR insomnia subtypes. Sleep, 34, 531-9.

Saper, C. B., Cano, G., \& Scammell, T. E. (2005). Homeostatic, circadian, and emotional regulation of sleep. The Journal of Comparative Neurology, 493, 92 - 98.

Schneider, C., Fulda, S., \& Schulz, H. (2004). Daytime variation in performance and tiredness/sleepiness ratings in patients with insomnia, narcolepsy, sleep apnea and normal controls. Journal of Sleep Research, 13, 373 - 383.

Segerstrom, S. C., Tsao, J. C. I., Alden, L. E., \& Craske, M. G. (2000). Worry and rumination: Repetitive thought as concomitant and predictor of negative mood. Cognitive Therapy and Research, 24, $671-688$.

Seidel, W. F., Ball, S., Cohen, S., Patterson, N., Yost, D., Dement, W. C. (1984). Daytime alertness in relation to mood, performance, and nocturnal sleep in chronic insomniacs and noncomplaining sleepers. Sleep, 7, $230-238$.

Shekleton, J. A., Rogers, N. L., \& Rajaratnam, S. M. (2010). Searching for daytime impairments of primary insomnia. Sleep Medicine Reviews, 14, 47 - 60 . 
Sierra, J. C., Guillen-Serrano, V., \& Santos-Inglesias, P. (2008). Insomnia severity index: Some indicators about its reliability and validity on an older adults sample. Revista de Neurologia, 47, $566-570$.

Smith, M. T., Huang, M. I., \& Manber, R. (2005). Cognitive behavioural therapy for chronic insomnia occurring within the context of medical and psychiatric disorders. Clinical Psychology Review, 25, $559-592$.

Smith, S. S. \& Trinder, J. (2001). Detecting insomnia: Comparison of four self-report measures of sleep in a young adult population. Journal of Sleep Research, 10, $229-235$.

Spielman, A. J., \& Glovinsky, P. B. (1991). The varied nature of insomnia, In P. Hauri (Ed.), Case studies in insomnia (pp. 1 -18). New York: Plenum Medical Book.

Spielman, A. J. \& Saskin, P. \& Thorpy, M. J. (1987). Treatment of chronic insomnia by restriction of time in bed. Sleep, 10, $45-56$.

Spitzer, R. L., Williams, J. B. W., Gibbons, M., \& First, M. B. (1996). Instruction manual for the structured clinical interview for DSM-IV (SCID-IV). New York: Biometrics Research Department, New York Psychiatric Institute.

Stepanski, E., Zorick, F., Roehrs, T. \& Roth, T. (2000). Effects of sleep deprivation on daytime Sugerman, J. S., Stern, J. A., \& Walsh, J. K. (1985). Daytime alertness in subjective and objective insomnia: Some preliminary research findings. Biological Psychiatry, 20, 741 725.

Tassi, P. \& Muzet, A. (2000). Sleep inertia. Sleep Medicine Reviews, 4, 341 - 353.

Taylor, S., \& Rachman, S. J. (1992). Fear and avoidance of aversive affective states: Dimensions and causal relations. Journal of Anxiety Disorders, 6, $15-25$. 
Thomsen, D. K., Mehlsen, M. Y., Christensen, S., \& Zachariae, R. (2003). Ruminationrelationship with negative mood and sleep quality. Personality and Individual Differences, 34, 1293 - 1301.

Ustinov, Y., Lichstein, K. L., Vander Wal, G. S., Taylor, D. J., Riedel, B. W., \& Bush, A. J. (2010). Association between report of insomnia and daytime functioning. Sleep Medicine, 11, $65-68$.

Valko, P. O., Bassetti, C. L., Bloch, K. E., Held, U., \& Baumann, C. R. (2008). Validation of the fatigue severity scale in a Swiss cohort. Sleep, 31, $1601-1607$.

Vallières, A., Ivers, H., Bastien, C. H., Beaulieu-Bonneau, S., \& Morin, C. (2005). Variability and predictability in sleep patterns of chronic insomniacs. Journal of Sleep Research, 14, $447-453$.

van Hilten, J. J., Braat, E. A., van der Velde, E. A., Middelkoop, H. A., Kerkhof, G. A., \& Kamphuisen, H. A. (1993). Ambulatory activity monitoring during sleep: An evaluation of internight and intrasubject variability in healthy persons aged 50-98 years. Sleep, 16, $146-150$.

Vitiello, M. V., Moe, K. E., \& Prinz, P. N. (2002). Sleep complaints cosegregate with illness in older adults: Clinical research informed by and informing epidemiological studies of sleep. Journal of Psychosomatic Research, 53, 555-559.

Walker, E. A., Katon, W. J., Jemelka, R. P. (1993). Psychiatric disorders and medical care utilization among people in the general population who report fatigue. Journal of General Internal Medicine, 8, 436 - 440 .

Webb, W. B. (1988). An objective behavioral model of sleep. Sleep, 11, 488-496. 
West, S. G., Finch, J. F., \& Curran, P. J. (1995). Structural equation models with nonnormal variables: Problems and remedies. In R. H. Hoyle (Ed.), Structural equation modeling: Concepts, issues and applications (pp. 56-75). Thousand Oaks, CA: Sage.

Wicklow, A., \& Espie, C. A. (2000). Intrusive thoughts and their relationship to actigraphic measurement of sleep: Towards a cognitive model of insomnia. Behavioral Research and Therapy, 38, $679-699$.

Williams, J., \& MacKinnon, D. P. (2008). Resampling and distribution of the product methods for testing indirect effects in complex models. Structural Equation Modeling, 15, 23-51.

Wohlgemuth, W. K., Edinger, J. D., Fins, A. I., \& Sullivan, R. J., Jr. (1999). How many nights are enough? The short-term stability of sleep parameters in elderly insomniacs and normal sleepers. Psychophysiology, 36, 233-244.

Wolpe, J. \& Lang, P.J. (1964). A fear schedule for use in behavior therapy, Behaviour Research and Therapy, 2, 27-30.

Zanarini, M. C., Skodol, A. E., Bender, D., Donal, R., Sanislow, C., Schaefer, E., ... Ghunderson, J. G. (2000). The collaborative longitudinal personality disorders study: Reliability of Axis I and II diagnoses. Journal of Personality Disorders, 14, 291 - 299.

Zhang, B., Wing, Y. K. (2006). Sex differences in insomnia: A meta-analysis. Sleep, 29, 85 - 93. 